\title{
CLEAN GASOLINE REFORMING WITH SUPERACID CATALYSTS
}

Final Technical Report for the Period September 25, 1990-September 24, 1992

\author{
By \\ Robert A. Keogh \\ Dennis E. Sparks \\ R. Srinivasan \\ Burtron H. Davis
}

Contract NO.: DE-AC22-90PC90049

For

U.S. Department of Energy

Pittsburgh Energy Technology Center

Pltsburgh, Pennsylvania

\author{
By \\ Universtty of Kentucky \\ Center for Applied Energy Research \\ 3572 Iron Works Pike \\ Lexington, Kentucky 40511
}


Title: CLEAN GASOLINE REFORMING WITH SUPERACID CATALYSTS

P.I.: Robert A. Keogh, Dennis E. Sparks, R. Srinivasan, and Burtron H. Davis, Center for Applied Energy Research, University of Kentucky, 3572 Iron Works Pike, Lexington, KY 40511

CONTRACT NO.: \#DE-AC22-90PC90049

PERIOD OF

PERFORMANCE: September 25, 1990 through September 24, 1992

OBJECTIVES:

The objectives of this project are to: (a) determine if a coal-derived naphtha can be hydrotreated to produce a product with a sufficiently low heteroatom content that can be used for reforming, (b) identify hydrocarbon compounds in the naphtha with concentrations greater than 0.5 wt.\%, (c) develop a $\mathrm{Pt} / \mathrm{Al}_{2} \mathrm{O}_{3}$ heavily chlorided catalyst and determine the activity, selectivity and deactivation of this catalyst using model compounds and the hydrotreated naphtha, and (d) develop both a sulfated $\mathrm{Pt} / \mathrm{ZrO}_{2}$ and $\mathrm{Fe} / \mathrm{Mn} / \mathrm{ZrO}_{2}$ catalyst formulations and determine the activity, selectivity and deactivation of these catalysts using model compounds and if warranted, the hydrotreated naphtha.

\section{Hydrotreatment}

\section{Naphtha Feedstocks}

Three, 55-gallon drums of an Illinois \#6 and a Black Thunder coal derived naphtha were recieved from the Wilsonville, Alabama, Advanced Integrated Two Stage Liquefaction pilot plant. The samples were obtained from V-161 (T-105 OH) during Run 260 (Black Thunder) and Run 261 (Illinois \#6). The location of V-161 in a simplified process flow diagram is shown in Figure 1. The elemental analyses of the as-received naphtha samples are given in Table 1. 


\section{Pilot Plant Operations}

The naphtha samples were hydrotreated in the reconfigured CAER $1 / 8$ tpd liquefaction pilot plant. The process diagram is shown in Figure 2. The plant consists of the following sections: (a) delivery section for naphtha, $\mathrm{N}_{2}$ and $\mathrm{H}_{2}$ (b) fixed bed, down-flow reactor, and (c) high and low temperature and pressure separators. Catalysts

Two commercially available catalysts were employed in this study. The first catalyst used was an American Cyanamid HDS 1442A (1/16" diameter, ca. 1/4" length). This Co-Mo alumina based catalyst was from the same batch that was used during the last run of the H-Coal pilot plant at Catlettsburg, Kentucky. The second catalyst was a Akzo KF-840 1.3Q Ni-Mo alumina catalyst. Analyses of the catalysts are given in Table 2. Prior to being loaded into the reactor, both catalysts were washed with acetone and dried in air overnight. The catalysts were calcined in air using a temperature of $325^{\circ} \mathrm{C}$ for 8 hours. One kilogram of each catalyst was used for the hydrotreating runs.

Sulfidation

The catalysts were sulfided in-situ using a mixture of 3.01 mole $\% \mathrm{H}_{2} \mathrm{~S}$ in hydrogen prior to the start of naphtha feed to the plant. The gas flow was $30 \mathrm{ft}^{3} / \mathrm{hr}$ during the sulfiding operations. The reactor temperature was increased rapidly from ambient to $175^{\circ} \mathrm{C}$ and then increased to a target temperature of $375^{\circ} \mathrm{C}$ at a rate of $25^{\circ} \mathrm{C} / \mathrm{hr}$. The composition of the process gas stream was continuously monitored during the sulfidation period by an on-line gas chromatograph. The $\mathrm{H}_{2} \mathrm{~S}$ concentration in the process gas stream for the sulfidation of both catalysts are shown in Figure 3 . 
The $\mathrm{H}_{2} \mathrm{~S}$ concentration became a constant value (ca. 3 mole \%) during the temperature ramp indicating that $\mathrm{H}_{2} \mathrm{~S}$ was no longer reacting with the catalyst. At this point, the temperature was increased to $375^{\circ} \mathrm{C}$ and held at this temperature for 90 minutes. The reactor temperature was then lowered to $300^{\circ} \mathrm{C}$ and the gas was changed to hydrogen (flow rate of $5 \mathrm{ft}^{3} / \mathrm{hr}$ ) prior to naphtha processing.

The reaction conditions used in the three hydrotreating runs are given in Table 3. The first run processed two barrels of the Black Thunder naphtha. The steadystate product was continuously collected in a barrel which was blanireted with nitrogen. After processing two barrels of the as-received naphtha, the run was terminated and the plant was purged with nitrogen and placed under a system pressure of $\mathbf{3 6 0}$ psig.

The feedstock for the second run was the product barrels obtained from the initial hydrotreating run. The run conditions for the second pass of the Black Thunder were the same as the first pass. Two barrels of product were obtained from this run.

Analysis of the Black Thunder product from the second pass indicated that an additional iun would be required to obtain a naphtha with a low nitrogen content. At this point, the Ni-Mo catalyst was loaded into the reactor and sulfided in-situ as described above. The feedstock for this run was the product obtained from the second run. The run conditions were the similar to these previously employed. The plant was run for 127 hours using the Black Thunder naphtha as the feedstock.

The feed was switched to the as-received Illinois \#6 naphtha after completion of the Black Thunder processing. During the processing of the Illinois \#6 naphtha, several process variables were changed and these are documented in Table 3. 


\section{Black Thunder Hydrotreatment}

The reason for the three processing runs of the Black Thunder can be seen in Figure 4 which shows the nitrogen content of the daily samples from each of the runs. The data in this figure clearly show the difficulty in removing nitrogen from the Black Thunder naphtha. The first pass removed only $43 \%$ of the nitrogen. The second and third pass removed $72 \%$ and $89 \%$ of the nitrogen, respectively, based on the nitrogen content of the as-received naphtha. The nitrogen content of the product from the third pass was $383 \mathrm{ppm}$, which was significantly higher than the < $10 \mathrm{ppm}$ target value. Based on the nitrogen removal data obtained from the three runs, it wouid require a total of ten passes to obtain a Black Thunder naphtha which has a nitrogen content of 2 ppm (Figure 5).

The difficulty in removing the nitrogen from the Black Thunder naphtha was further investigated by determining the amount of basic nitrogen (e.g., anilines, quinolines, etc.) and non-basic nitrogen compound (e.g., indoles) in the first and second pass products. The basic nitrogen compounds were extracted from the asreceived, the first and second pass product naphtha samples with $10 \% \mathrm{H}_{3} \mathrm{PO}_{4}$. The concentration of the basic compounds of the feed and the products from the first two passes is given in Figure 6. The results indicate that a substantial concentration of the basic nitrogen compounds are present in the Black Thunder product after the second pass. The second pass only removed 54 wt.\% of the basic nitrogen compounds from the as-received naphtha.

The nitrogen content of the extracted feed and product naphtha samples are given in Figure 7. The data indicate that the neutral nitrogen compound class has a 
small contribution to the total nitrogen content of the samples. These data also suggest that the difficulty in removing the nitrogen from the Black Thunder naphtha was not due to the neutral nitrogen compounds. Therefore, it was concluded that the basic nitrogen compounds in this naphtha was responsible for the high nitrogen content in the hydrotreated products.

The oxygen content of the feed and processed samples are shown in Figure 8. Although the oxygen content of the as-received Black Thunder naphtha was high (2.01 wt.\%), it was easily removed when compared to the removal of nitrogen. The first hydrotreatment ril noved $96 \%$ of the oxygen. Further processing reduced the oxygen content to $0.03 \mathrm{wt} . \%$ for the third pass. This value was close to the detection limit of the FNAA riethod (.02 wt.\%); thus the actual concentration of the oxygen may be signifirantly lower than the reported value. Therefore, the data suggested that the hydrotreatment of the Black Thunder naphtha yielded a product with an oxygen content which met the target specification of $<10$ ppm oxygen.

Removal of sulfur from the Black Thunder naphtha was also easier to remove than the nitrogen. The sulfur contents of the feed and hydrotreated samples are given in Figure 9. The first pass removed $93 \%$ of the sulfur. Further hydrotreatment removed $98 \%$ of the sulfur from the feed. However, to obtain a product which contains < 10 ppm S, the Black Thunder naphtha had to be processed twice.

The heteroatom removal data obtained from the processing of the Black Thunder naphtha suggested a change in process parameters would be required to obtain a product which would meet the target specifications ( $<10 \mathrm{ppm}$ ) of 
heteroatom removal. The change in process parameters investigated during the hydrotreatment of the Illinois \#6 are documented in Table 2.

Illinois \#6 Naphtha Hydrotreatment

The hydrotreatment of the llinois \#6 naphtha was initiated after the Black Thunder naphtha was hydrotreated for the third time. A sufficient number of plant turnovers were completed prior to collection of the product.

Both the weight hourly space velocity (WHSV) and reactor temperature were varied during the processing of the Illinois \#6 naphtha with the Ni/Mo catalyst. Figure 10 shows the effect of these parameters on the nitrogen content of the product. The data indicated that a hydrotreated product which meets the $<10 \mathrm{ppm}$ target value can be obtained; however, a low space velocity $(0.3)$ and a high reactor temperature $\left(403^{\circ} \mathrm{C}\right)$ were required to obtain a nitrogen content of $3.1 \mathrm{ppm}$ in the product.

The oxygen contents of the feed and product samples are given in Figure 11. As can be seen in the figure, an oxygen content of 0.03 wt.\% (91.4\% HDO) was obtained for the naphtha product using the high severity conditions. As stated above, the $0.03 \%$ oxygen values is close to the detection limit of the method and may be much lower than $0.03 \%$ reported. It appears that oxygen was easily removed from the Illinois \#6 naphtha and was comparable to the values obtained from the hydrotreated Black Thunder naphtha.

The sulfur contents of the feed and product samples are given in Figure 12. The sulfur content of the product barrel naphtha using the most severe conditions was $33 \mathrm{ppm}(90.7 \% \mathrm{HDS})$. The relatively high sulfur content obtained using the most severe processing condition did not meet the target value of $<10 \mathrm{pm}$. However, as 
can be seen in Figure 12, at the lower reactor temperatures and using the Co/Mo catalyst, the sulfur content of the processed naphtha were $3.3 \mathrm{ppm}\left(352^{\circ} \mathrm{C}\right.$, WHSV $=$ 0.8 ) and $5.4 \mathrm{ppm}\left(351^{\circ} \mathrm{C}, \mathrm{WHSV}=0.2\right)$. These data suggest that for the Ni/Mo catalyst, different operating conditions would be required to obtain the same low sulfur contents of the naphtha is that obtained with the Co/Mo catalyst.

A comparison of HDO and HDN (Figure 13) indicates that for the Black Thunder naphtha, the oxygen compounds can be removed much easier than the nitrogen compounds using both catalysts. For the llinois naphtha, which was only processed using the Ni/Mo catalyst, a low WHSV (0.3) was required to obtain a level of HDN which approximates the level of HDO. A comparison of the HDS and HDN (Figure 14) show essentially the same trends discussed above for Figure 13.

A comparison of HDO and HDS obtained in these runs are shown in Figure 15. The data presented in this figure show that high levels of HDO and HDN (>90\%) were obtained for all the hydrotreatment runs and that the levels of HDO and HDS were approximately equal for each run.

\section{Identification of Hydrocarbons by GCIMS}

Identifications of the hydrocarbon compounds in the hydrotreated naphtha samples are given in Table 4. Identifications were made on the basis of the injection of standard compounds and mass spectroscopy. The major components in the Illinois \#6 and Black Thunder naphtha samples were cycloalkanes (cyclopentanes and cyclohexanes). Methylcyclohexane was the major component for both naphtha samples. Based on these data, methylcyclohexane, cyclohexane, methylcyclopentane 
and n-pentane will be used as model ccmpounds in the evaluation of the activity of the sulfated $\mathrm{Pt} / \mathrm{ZrO}_{2}$ and the chlorided $\mathrm{Pt} / \mathrm{Al}_{2} \mathrm{O}_{3}$ catalysts.

\section{Chloridation of $\mathrm{Pt} / \mathrm{Al}_{2} \mathrm{O}_{3}$ Catalysts}

Two aluminas, one a commercially available pellet (S.A. $\left.=196 \mathrm{~m}^{2} / \mathrm{g}\right)$ and one which was made in-house (S.A. $=149 \mathrm{~m}^{2} / \mathrm{g}$ ), were used in this project. The alumina powder was made by precipitating $\mathrm{AlCl}_{3} \cdot 6 \mathrm{H}_{2} \mathrm{O}\left(.5 \mathrm{M}\right.$ in $\mathrm{Al}$ ) with $\mathrm{NH}_{4} \mathrm{OH}$ to a $\mathrm{pH}$ of 7.6. The gel was copiously washed with distilled $\mathrm{H}_{2} \mathrm{O}$ and dried. The dried alumina was calcined at $500^{\circ} \mathrm{C}$ in air for 16 hours. Platinum was impregnated on the aluminas with chloroplatinic acid. An acetone solution containing the desired concentration of Pt was added to the alumina carrier and stirred overnight. The acetone was removed by rotoevaporation. The catalyst was dried overnight and stored in a dessicator. Surface areas were determined by the BET method.

A plug flow reactor system was used for reduction, chloridatic nand activity testing of the catalysts.

Chloride analysis were done using standard ion chromatography techniques.

Methylcyclohexane and n-pentane were obtained commercially and dried using molecular sieves (3A) prior to use. All gases $\left(\mathrm{N}_{2}\right.$ and $\left.\mathrm{H}_{2}\right)$ were ultra high purity (99.999\%) and passed over molecular sieves prior to introduction into the reactor system. The naphtha sample was obtained from the Wilsonville Advanced Liquefaction Pilot Plant. The llinois \#6 naphtha was hydrotreated in the CAER PIPU pilot plant prior to use with the chlorided $\mathrm{Pt} / \mathrm{Al}_{2} \mathrm{O}_{3}$ catalyst. 


\section{Chloride Pretreatment}

In the initial set of chloriding experiments, the Pt impregnated alumina powder (S.A. $\left.=149 \mathrm{~m}^{2} / \mathrm{g}\right)$ catalysts were used. The catalyst was reduced prior to the pretreatment using a temperature of $450^{\circ} \mathrm{C}$ and $260 \mathrm{psig} \mathrm{H}_{2}(100 \mathrm{sccm} / \mathrm{min}$. overnight. Chloriding was done at $300^{\circ} \mathrm{C}$ using nitrogen at 250 psig pressure (100 sccm/min.) and $\mathrm{CCl}_{4}$ as the chloriding agent. The $\mathrm{CCl}_{4}$ partial pressure in the gas phase was controlled by the temperature of the vessel containing liquid $\mathrm{CCl}_{4}$ that was used to saturate the nitrogen stream. Gas samples were taken to monitor the $\mathrm{CO}_{2}$ content of the product gas by gas chromatography. In addition, gas samples were analyzed in the region of maximum $\mathrm{CO}_{2}$ production and in the initial stage of chloridation by GC/MS. No $\mathrm{COCl}_{2}$ or $\mathrm{CO}$ were found in the product gas stream in any of the samples using nitrogen as the carrier. The only compounds detected were $\mathrm{N}_{2}$, $\mathrm{CO}_{2}$, and at the enc of the final time period of chloridation, $\mathrm{CCl}_{4}$. Thus, $\mathrm{CCl}_{4}$ reacts with alumina to produce $\mathrm{CO}_{2}$ as the only carbon containing product; however, it is not known whether the chloride containing species is $\mathrm{AlOCl}, \mathrm{AlCl}_{3}$, or some more complex criloroalumina compound is formed. However, it appears that chloriding occurs in steps with the firct product being an alumina oxy chloride followed by subsequent formation of $\mathrm{AlCl}_{3}$ ur other volatile $\mathrm{Al} / \mathrm{Cl}$ containing species. Thus, for discussion purposes the reaction is written in two steps:

$$
\begin{gathered}
2 \mathrm{Al}_{2} \mathrm{O}_{3}+\mathrm{CCl}_{4}-4 \mathrm{AlOCl}+\mathrm{CO}_{2} \\
2 \mathrm{AlOCl}+\mathrm{CCl}_{4}-2 \mathrm{AlCl}_{3}+\mathrm{CO}_{2} \\
\left.\left.\mathrm{~A} / \mathrm{Cl})_{3}\right)_{\text {ads }}=\mathrm{AlCl}\right)_{\text {gas }}
\end{gathered}
$$


The four $\mathrm{Pt} / \mathrm{Al}_{2} \mathrm{O}_{3}$ catalysts were chlorided using this method. A typical $\mathrm{CO}_{2}$ generation curve obtained using the $.4 \mathrm{wt} . \% \mathrm{Pt}_{\mathrm{Al}} \mathrm{O}_{3}$ catalyst is shown in Figure. 16 . Because of the dead volume between the $\mathrm{CCl}_{4}$ saturator and the catalyst bed, the time taken as zero does not coincide with the time that $\mathrm{CCl}_{4}$ first contacts the catalyst bed. As can be seen in this figure, the $\mathrm{CO}_{2}$ generated goes through a maximum at .31 mole \% in 90 minutes. The low amount of $\mathrm{CO}_{2}$ generated, which was typical for this set of catalysts, suggested that the amount of chloride added would be lower than the 8-13 wt.\% required for an active isomerization catalyst. Analysis of the catalyst indicated that the chloride content was $3.66 \pm .30 \mathrm{wt} . \%$. The chloride content of these Pt/Al $\mathrm{O}_{3}$ catalysts did not exceed $4 \mathrm{wt} . \%$ and appeared to correlate with the maximum $\mathrm{CO}_{2}$ content generated during the chloriding pretreatment.

The next set of experiments performed in an attempt to increase the chloride content of the catalyst employed commercially available $\mathrm{Al}_{2} \mathrm{O}_{3}$ pellets. The method for the chloriding pretreatment was also changed. Nitrogen was used for the $\mathrm{CCl}_{4}$ entrainment at atmospheric pressure and $100 \mathrm{sccm} / \mathrm{min}$. The reactor temperature remained the same $\left(300^{\circ} \mathrm{C}\right)$. The $\mathrm{CO}_{2}$ concentrations generated during the chloridation of the alumina pellets are shown in Figure 17. As can be seen, the amount of $\mathrm{CO}_{2}$ produced was significantly greater than the amount shown in Figure 16. The maximum $\mathrm{CO}_{2}$ generated $(23.2$ mole \%) was also obtained at a shorter reaction time (10 $\mathrm{min}$.$) . The chloride content of the catalyst was determined to be$ $13.70 w t . \%$. These data suggested that the observed increase in chloride added to the alumina pellets may be due to the form of the catalyst (pellets vs. powder). 
In the next chloriding experiment, the alumina pellets were ground to -100 mesh (S.A. $=200 \mathrm{~m}^{2} / \mathrm{g}$ ) to determine if the form of the catalyst affects the amount of chloride retained by the alumina. The powdered alumina was chlorided using the same procedure as that used for the alumina pellets. The $\mathrm{CO}_{2}$ generated during the chloridation of this sample is shown in Figure 17. As can be seen, the maximum $\mathrm{CO}_{2}$ content is lower and a longer reaction time was required to obtain it. This correlates with the lower chloride content (5.79 wt.\%) of the powdered catalyst. One explanation for the data shown in Figure 17 is that for the alumina pellets, the $\mathrm{CCl}_{4}$ penetrates the pellet and reacts rapidly (reactions [1] and [2]) with the alumina to produce a chlorided alumina compound and produce $\mathrm{CO}_{2}$. The penetration of $\mathrm{CCl}_{4}$ into the pellet and the escape of the $\mathrm{CO}_{2}$ out of the pellet was not believed to be diffusion limited. It is believed that the diffusio $\mathrm{ICl}_{3}$ from the interior of the catalyst or particle is slow because of diffusion, or a process that resembles diffusional limitations. Thus, it is viewed that $\mathrm{AlCl}_{3}$ forms uniformly throughout the catalyst particle or pellet but that it is iost from the catalyst bed by desorption from the surface boundary of the catalyst particle or pellet. The geometric surface boundary of the pellet is much smaller than that of the same amount of catalyst when it is present in powder form. Hence, it is believed that the rate of chloridation is similar in both the powder and the pellet. However, desorption to the gas in the void volume and transport from the catalyst bed is much slower for the pellet than for the powder.

With the development of a method of chloride addition which produced an alumina with a sufficiently high chloride content, the next set of experiments used the alumina pellets impregnated with $2 \mathrm{wt. \%}$ Pt. This catalyst was reduced as described 
above and chiorided using the same procedure as in the alumina pellet experiments. In addition this catalyst was also chlc ded without prior reduction. The chloride content of the resulting catalysts are shown in Figure 18. The data shown in Figure 18 indicate that the addition of Pt to the alumina pellets decreases the chloride content of the catalysts when compared to that of the alumina pellets. The reduced catalyst produced a slightly higher chloride content than the catalyst which was not reduced prior to chloridation. Without further data, it is not possible to unambiguously determine the cause for the lower chloride content; however, the data does suggest that the impregnation of the alumina with the Pt has removed a number of sites that react with the $\mathrm{CCl}_{4}$.

The data presented above indicate that a $2 \% \mathrm{Pt}_{\mathrm{A}} \mathrm{Al}_{2} \mathrm{O}_{3}$ (pellet) can be chlorided to produce a catalyst containing a high chloride content $(7.22 \mathrm{wt. \% )}$ and that the extent of chloride addition can be monitored by the amount of $\mathrm{CO}_{2}$ produced during this pretreatment. The reproducibility of this method is illustrated in Figure 19 which shows the $\mathrm{CO}_{2}$ generation curves for a number or chloride pretreatments. Note that in Test \#11, the chloriding pretreatment was terminated just after the $\mathrm{CO}_{2}$ production reached a maximum. The resulting catalyst had a $5.93 \mathrm{wt} . \%$ chloride content. This run suggests that using this method of monitoring, the extent of chloridation can accurately produce a series of catalysts with varying chloride contents. Isomerization Activity

The isomerization activities of the $2 \% \mathrm{Pt}_{\mathrm{Al}} \mathrm{O}_{2} \mathrm{O}_{3}$ catalysts which have different chloride contents are shown in Figure 20. For all of the reaction temperatures studied, the $\mathrm{Pt} / \mathrm{Al}_{2} \mathrm{O}_{3}$ (pellet) catalyst containing $7.22 \mathrm{wt} . \% \mathrm{Cl}$ affected significantly higher 
conversions of it-pentane. The product distribution obtained for both of these catalysts were similar. The major product of the runs was isopentane (>99\%). In addition, minor amounts (ca. $0.1 \cdot 0.2 \mathrm{wt}$.\%) of isohexanes were also produced. Very little hydrogenolysis was observed for both of the catalysts. The data obtained from these isomerization runs with $n$-pentane suggest that the higher conversions are obtained with catalysts with higher chloride concentrations.

The chlorided $2 \mathrm{wt} . \% \mathrm{Pt}_{\mathrm{A}} \mathrm{I}_{2} \mathrm{O}_{3}$ (pellet) was also tested with methylcyclohexane (MCH) using a number of reactor temperatures. The results indicated that although conversion of MCH: were relatively high, the major products were 2-carbon cyclopentanes (ca. $95^{+}$ivt.\%) which indicated that little ring-opening of the $\mathrm{MCH}$ was accomplished. In an attempt to change the selectivity of the catalysts for the conversion of $\mathrm{MCH}$, the total hydrogen pressure of 1 ne reactor was increased while holding the temperature constant. The effect of pressure on the conversion of $\mathrm{MCH}$ is shown in Figure 21. Increasing the reactor pressure from 100 to 730 psig slightly increases the conversion from 60 to $80 \mathrm{wt}$ \%. However, the increase in pressure significantly changes the product distribution (Figure 22). The 2-carbon cyclopentanes, which are the major products of the conversion of $\mathrm{MCH}$ at lower pressures, significantly decrease in concentration with increasing pressure. It appears from these data that in order to obtain ring opening at lower temperatures, higher reactor pressures are required.

A hydrotreated naphtha derived from processing an Illinois \#6 coal at Wilsonville was obtained as a reforming feedstock using the chlorided $2 \% \mathrm{Pt} / \mathrm{Al}_{2} \mathrm{O}_{3}$ 
(pellet) catalyst. The catalyst exhibited an initial high activity in producing branched alkanes; however, this activity decreased rapidly with time on stream.

\section{Effect of Pt Concentration on Isomerization}

Four catalysts containing different Pt concentrations were prepared to determine the effect of Pt on the activity for the isomerization of n-pentane and cycloperitane. The catalysts were reduced in flowing hydrogen as previously reported prior to chloridation. The catalyst was chlorided using $\mathrm{CCl}_{4}$ entrained in nitrogen as previously reported. All of the pretreatment steps were done in-siru. Chloridation of the catalyst was terminated when the $\mathrm{CO}_{2}$ concentration in the product gas stream reached a maximum. Upon termination of the chloriding pretreatment, the reactors were purged with $\mathrm{H}_{2}$ and the reactor temperature was set in preparation for liquid feed.

The isomerization of $n$-pentane using these four catalysts was studied using a WHSV of 1 , a hydrogen pressure of $250 \mathrm{psig}$ and three reaction temperatures $\left(150^{\circ} \mathrm{C}\right.$, $200^{\circ} \mathrm{C}$, and $250^{\circ} \mathrm{C}$ ). The conversions obtained for these runs are shown in Figure 23. At a reaction temperature of $150^{\circ} \mathrm{C}$, the maximum conversion ( $22 \mathrm{wt} . \%$ ) is obtained with the catalyst containing 2 wt.\% Pt. The data in this figure suggest the same trend will be obtained for a reaction temperatures of $200^{\circ} \mathrm{C}$ and $250^{\circ} \mathrm{C}$. The data from these experiments indicate that the concentration of Pt on the catalyst can significantly effect the conversion level of $n$-pentane, providing the conversions are not at or near equilibrium. For all of these experiments, the major product of the reaction was isopentane. Very little hydrogenolysis was observed in the reactions using the four catalysts. 
The isomerization of cyclopentane was also studied rsing these catalysts and the data are shown in Figure 24. Although not all the catalysts were run, the data does suggest a different trend than that obtained using n-pentane as the feedstock. At the lower reaction temperatures $\left(150^{\circ} \mathrm{C}, 200^{\circ} \mathrm{C}\right.$, and $\left.250^{\circ} \mathrm{C}\right)$, there were no significant differences in the conversions using the catalysts which contain $0.4 \mathrm{wt} . \%$ and $1.0 \mathrm{wt} . \% \mathrm{Pt}$. The conversions for these two catalysts at these tempe atures are also low ( $<10 \mathrm{wt. \%})$. At the higher temperatures $\left(300^{\circ} \mathrm{C}\right.$ and $\left.350^{\circ} \mathrm{C}\right)$, the conversion of iyclopentane increases with increasing temperature. This is similar to the results obtained by Beltramini et al. (in Catalyst Deactivation, 1991, C. H. Bartholomew and J. B. Butt, eds., Elsevier Science Pub., B.V., Amsterdam). They suggested that coke deposition increased with increasing Pt concentration.

\section{The Effect of Aromatics on the Activity and Selectivity of $\mathrm{Pt} / \mathrm{Al}_{2} \mathrm{O}_{3} / \mathrm{Cl}$ Catalysts}

The effect of aromatics on the selectivity and activity of the chlorided $\mathrm{Pt}_{\mathrm{Al}} \mathrm{O}_{3}$ catalyst was investigated using a 10:90 ethylbenzene/ $\mathrm{MCH}$ mixture as a feedstock. A comparison of the conversions obtaiñed with this mixture and $100 \% \mathrm{MCH}$ as a function of pressure is shown in Figure 25. The data show that the addition of the aromatic had no effect on the activity of the catalyst. The ethylbenzene was almost entirely converted to ethylcyclohexane which was converted to a 2-carbon cyclohexane.

An additional run using only ethylbenzene as the feedstock was made to further investigate the effect of aromatics on the activity of this catalyst. The results from this run are shown in Figure 26. As can be seen, the conversion of ethylbenzene was high during the entire run (>90 wt.\%). The primary products were ethylcyclohexane and dimethylcyclohexane. As the time on stream increased, the ethylbenzene 
conversion remained fairly constant; however, the concentration of ethylcyclohexane significantly increased. These data suggested that as the catalyst aged, the metal function (hydrogenation) did not deactivate. The deactivation was due to loss of acidity.

The State of Platinum in Pt/Al $\underline{O}_{3}$ Catalysts Containing High Chloride Loading

Two materials were utilized in this study. A high surface area $\left(230 \mathrm{~m}^{2} / \mathrm{g}\right)$ commercially available alumina was impregnated with an acetone solution of chloroplatinic acid to produce a catalyst containing $4 \mathrm{wt} . \% \mathrm{Pt}$.

An alumina precipitated by bubbling carbon dioxide into a potassium aluminate solution after calcination at $600^{\circ} \mathrm{C}$ in air; this alumina had a surface area of $200 \mathrm{~m}^{2} / \mathrm{g}$ and contained about $0.5 \mathrm{wt} . \% \mathrm{~K}$. The alumina was impregnated with chloroplatinic acid to produce a material containing $10 \mathrm{wt} . \% \mathrm{Pt}$.

The XPS analyses were carried out in Leybold-Hereaus LH-11 spectrometer equipped with a heatable sample probe and reaction chamber. The catalyst was pressed into a thin wafer and studied in the as-received state, reduced in flowing $\mathrm{H}_{2}$ at $300^{\circ} \mathrm{C}$ for one hour, and chlorided by a flow of He saturated with $\mathrm{CCl}_{4}$ at the same conditions in that order. The Al2p, Pt4f, C1s, O1s, Cl2p regions of the samples were monitored. The alumina support was also examined for any discernible surface modification on exposure to chlorine. The data were acquired using MgKa (1253.6 $\mathrm{eV}$ ) excitation with a pass energy of $20 \mathrm{eV}$, were corrected for background and subsequently smoothed using the Savitsky-Golay algorithm. The smoothed spectra were curve-fitted to elucidate the relative area of the various chemical species by using gaussian peak shapes and a non-linear least squares method. The sensitivity factors 
employed in quantitation have been calculated for the instrument with typical analyzer settings and operating conditions.

For the $10 \% \mathrm{Pt}$ catalyst the XPS spectrum for the $\mathrm{Al} 2 \mathrm{p}$ and $\mathrm{Pt} 4 \mathrm{f}$ region is very broad and is readily deconvoluted to show the presence of $\mathrm{Pt}$ in the chlorided form. However, following in-situ reduction at $300^{\circ} \mathrm{C}$ for 1 hour in flowing hydrogen, an XPS spectrum showing peaks that are characteristic of $A l 2 p$ in alumina and Pt $4 f$ in the zero valence state is obtained. Thus, $\mathrm{Pt}$ can be distinguished in both the chlorlded and the metallic state at this high loading. Following in-situ chloriding at $300^{\circ} \mathrm{C}$ for 1 hour in flowing helium saturated with $\mathrm{CCl}_{4}$, there is essentially no change in the $\mathrm{Pt} 4 \mathrm{f}$ peak. Thus, chloriding did not convert the $\mathrm{Pt}^{\circ}$ to an oxidized state; the $\mathrm{Pt}$ remained reduced to the zero valent state.

The overlap of the chlorided Pt $4 f$ and the Al $2 p$ of alumina makes it difificult to obtain an accurate measure of full width at half maximum (FWHM) of the Al $2 p$ peak. However, this can be done for spectra for the reduced and the chlorided samples. For the reduced sample the FWHM is $1.94 \mathrm{eV}$. For the chlorided sample the Al2p peak FWHM is $3.25 \mathrm{eV}$ (or $2.29 \mathrm{eV}$ if the measurement is based upon the deconvoluted peak), and following reduction again the FWHM is 2.45 e.V.

The $10 \mathrm{wt} . \% \mathrm{Pt}$ alumina catalyst was reduced in flowing hydrogen at $450^{\circ} \mathrm{C}$ and was then chlorided in a flow-through reactor at $300^{\circ} \mathrm{C}$ in a nitrogen stream saturated with $\mathrm{CCl}_{4}$. The reaction was continued until the $\mathrm{CO}_{2}$ evolution had reached a maximum and then declined to a low value. Bulk chemical analysis indicated that the chloride level was about $7 \mathrm{wt} . \%$. The chlorided sample was pelleted and transferred to the XPS sample holder in a glovebox and consequently was studied by XPS with 
minimum exposure to air. The FWHM for the Al2p peak is $2.20 \mathrm{eV}$, in good agreement with the deconvoluted peak for the sample chlorided in the reactor chamber attached to the XPS instrument. The peak corresponding to $\mathrm{Pt}^{\circ}$ is clearly evident in this sample. From XPS measurements, the $\mathrm{Cl}$ fraction of the elements is 0.04 for the sample chlorided in the plug flow reactor and for the sample chlorided insitu, this fraction is $\mathbf{0 . 0 3}$.

For 10 wt.\% $\mathrm{PuAl}_{2} \mathrm{O}_{3}$ catalyst, it is observed that the fraction of $\mathrm{Cl}$ on the surface is 0.035 for the chlorided sample but the fraction decreases to 0.013 following treatment in hydrogen for 1 hour at $300^{\circ} \mathrm{C}$ (Table 5). We have reported previously that the surface chloride concentration decreases upon reduction with hydrogen, and the current data are in agreement with this observation.

The XPS spectrum of the as-prepared $4 \mathrm{wt} . \%$ Pt catalyst indicated that the Pt was present in an oxidized state and that it was included in the larger Al2p peak. Thus, the $\mathrm{Pt}$ was presumably present as $\mathrm{PtCl}_{6}{ }^{2+}$, or a similar compound of related stoichiometry. Following in-situ reduction in flowing hiydrogen for 1 hour at $300^{\circ} \mathrm{C}$, a peak corresponding to $\mathrm{Pt}^{\circ}$ was observed. In-situ chloriding of the sample in the XPS did not eliminate the peak corresponding to $\mathrm{Pt}^{\circ}$. The data with the 4 wt.\% $\mathrm{Pt}$ catalyst is similar to that obtained with the $10 \mathrm{wt} . \%$ Pt catalyst.

The chloride appears to be present in at least two chemical states in the asprepared, the chlorided, and in the re-reduced samples. It also appears that reduction in hydrogen following the chloriding reduces the surface concentration of the lower-binding, i.e., more-negative chloride. Thus, the present data is consistent with our earlier observation of a significant reduction in the surface chloride 
concentration following hydrogen reduction. Bulk analysis of the sample does not show a significant decrease in the chloride concentration following two oxidation and reduction cycles. These observations suggest that the more negative chloride ion migrates into the bulk of the alumina support with hydrogen reduction and diffuses to the surface in an oxygen atmosphere. Since chloride was not added during the oxidation portion of a cycle, it is clear that chloride migrates from the interior during oxidation; however, many cycles of oxidation and reduction would be required to distinguish between the removal of chloride to the gas phase and the migration into the bulk during hydrogen reduction.

When chloroplatinic acid was added to a silica support, the XPS data indicated that the chloroplatinum complex survived drying. However, reduction in hydrogen at $400^{\circ} \mathrm{C}$ converted the platinum to $\mathrm{Pt}^{\circ}$. Subsequent treatment with $\mathrm{CCl}_{4}$ converted part of the platinum to a more positive form, presumably a chloroplatinum cornpound. In the present study, the aluminum peak of the support prevents making an accurate measure of the amount of platinum in an oxidized state and $\mathrm{Pt}^{\circ}$. However, it does not appear that the addition of chloride from $\mathrm{CCl}_{4}$ and the subsequent reduction causes a measurable change in the amount of $\mathrm{Pt}^{\circ}$ from that of a reduced form of the catalyst.

In summary, it appears that the heavily chlorided ( $3 \mathrm{wt} . \%$ and higher) Ptalumina catalysts the $\mathrm{Pt}$ is present as $\mathrm{Pt}^{\circ}$, and not as a chloride. It also appears that the chloride is present in at least two chemical environments; furthermore, the chloride present on the lower binding energy form appears to be mobile. Thus, treatment of the chlorided catalyst causes the lower binding energy chloride concentration to decrease on the surface; subsequent treatment in oxygen causes bulk chloride to 
migrate to the surface. Silica is less amenable to chloriding so that on this support, in contrast to alumina, the treatment with $\mathrm{CCl}_{4}$ results in chloridation of the platinum.

\section{Zirconium Based Catalysts}

\section{Sulfated $\mathrm{Pt} / \mathrm{ZrO}_{2}$ Catalysts}

Hydrous zirconia was precipitated from a 1.14 liter, $0.5 \mathrm{M}$ zirconium tetrachloride (Alfa Products, Morton Thiokol) solution at a pH of 10.5 by rapidly adding 1.4 liters of $15 \mathrm{~N} \mathrm{NH}_{4} \mathrm{OH}$, while vigorously stirring. The resulting precipitate was washed with water by repeated filtration/reslurrying cycles until a negative test for $\mathrm{Cl}^{-}$ions was obtained. The filtered cake was dried at $120^{\circ} \mathrm{C}$ overnight.

The dried gel was crushed in a grinder and dried again at $120^{\circ} \mathrm{C}$ for an hour. This dried sample was sulfated using $10 \mathrm{~mL}$ of $1 \mathrm{~N} \mathrm{H}_{2} \mathrm{SO}_{4}$ per gram of $\mathrm{ZrO}_{2}$. The $\mathrm{ZrO}_{2}$ powders were added to a beaker containing the required amount of $1 \mathrm{~N} \mathrm{H}_{2} \mathrm{SO}_{4}$. The contents were stirred with a magnetic stirrer for 1 hour, after which the sulfated $\mathrm{ZrO}_{2}$ was filtered without any further washing. This sample was dried again at $120^{\circ} \mathrm{C}$ for 2 hours and then impregnated with $\mathrm{H}_{2} \mathrm{PtCl}_{6}$ so as to yield 0.6 wt.\% platinum. This sample was dried at $120^{\circ} \mathrm{C}$ overnight and then stored in a vacuum desiccator. The chemical analysis from this sample shows $0.57 \% \mathrm{Pt}$ and $2.56 \% \mathrm{~S}$.

The catalyst was activated at $725^{\circ} \mathrm{C}$ for 2 hours in a an air atmosphere prior to activity testing. The catalyst was placed in a dried $25 \mathrm{~mL}$ stainless steel microautoclave immediately after activation. The reactor containing the catalyst was cooled to ambient temperature in a desiccator prior to the addition of the feedstock. All feedstocks were dried using molecular sieves (3A). In a typical experiment, $2 \mathrm{~g}$ of catalyst and $4 \mathrm{~g}$ of feed were charged to the reactor. The reactor was flushed with 
hydrogen arid the final $\mathrm{H}_{2}$ charge was 1 atmosphere (ambient temperature). The $\mathrm{H}_{2} / \mathrm{MCH}$ mole ratio was 0.02 . The reactor was heated to $150^{\circ} \mathrm{C}$ using a fluidized sand bath and vertically shaken $(400 \mathrm{cpm})$ to facilitate mixing. It is calculated that greater than $90 \%$ of the methylcyclohexane should be in the liquid phase for the $2: 1$ ratio of feed:catalyst ( $2 \mathrm{~g}$ catalyst and $4 \mathrm{~g}$ feed). Upon completion of the experiment, the reactor was cooled to room temperature and vented. The catalyst was separated from the liquid products by filtration and the sample was stored in a freezer prior to analysis.

The analysis of the products was done using a capillary gas chromatograph on an FID detector. The capillary column installed was a 60 meter $\times .32 \mathrm{~mm}$ DB-5 (.32 $\mu \mathrm{m}$ film thickness). Identification of the components was done by GC/MS and standard compounds.

For a number of Pt-containing sulfated zirconia catalysts, we have found that the maximum methylcyclohexane (MCH) conversion (about 20 wt.\%) can be obtained in $15 \mathrm{~min}$. using a batch reactor and a reaction temperature of $150^{\circ} \mathrm{C}$ and $1 \mathrm{~atm}$. of $\mathrm{H}_{2}$, and no further reaction occurs during 120 minutes. The limit to the conversion is not due to thermodynamic limitations.

A series of experiments were completed using $\mathrm{Pt} / \mathrm{ZrO}_{2} / \mathrm{SO}_{4}$ catalysts to obtain data in an attempt to explain the reason for the limit to the conversion that was observed. In the first set of experiments a conversion versus residence time curve (Figure 27) was generated. The data shown in this figure indicated a conversion curve with reaction time that is typical of the catalyst preparations that were tested. It 
clearly demonstrates that the maximum conversion is less than 20 mole $\%$ whereas thermodynamic data indicate that greater than $\mathbf{5 0}$ mole \% conversion is possible.

In another set of experiments, the amount of reactor volume which was occupied by the catalyst and feedstock was varied to determine if a reaction taking place in the liquid or in the gas phase was limiting the conversion of $\mathrm{MCH}$. The $\mathrm{MCH} /$ catalyst ratio was held constant at 2:1. The data (Figure 28) show that the conversion remains constant until the reactor is essentially filled with liquid, and the low conversion for the nearly full vessel is considered to be due to poor mixing. It therefore does not appear that the observed maximum conversion is due to the reaction taking place only in the gas or the liquid phase.

Another set of experiments was conducted with this catalyst where the $\mathrm{MCH} /$ catalyst ratio was varied from 0.5 to 4.5 . The grams of $\mathrm{MCH}$ converted and the number of turnovers (molecules MCH converted per molecule sulfur in the activated catalyst) are shown in Figures 29 and 30 respectively. The number of turnovers shown in Figure 30 increases linearly as the $\mathrm{MCH} /$ catalyst ratio increases. This increase in the number of turnovers is not the result expected for a simple acid catalyzed reaction. Since the same amount of catalyst is used in all of the experiments (2g), the same number of active sites are present in each run. Therefore, the number of turnovers should be the same in all of the experiments independent of the amount of $\mathrm{MCH}$ used in the run. Since this is not the case, the results suggest that the catalyst is acting as an initiator and that an active species is absorbed into the liquid and/or gas phase where it continues the reaction. 
A mixture of $50 / 50$ wt.\% methylcyclopentane (MCP) and $\mathrm{MCH}$ was used as a feedstock to determine if the 2-carbon cyclopentanes formed from MCH were poisoning the catalyst. The conversion data obtained for the $50 / 50$ mixture, the pure MCP, and the MCH together with the calculated MCP and $\mathrm{MCH}$ conversions are shown in Figure 31. As can be seen, the addition of MCP to the feedstock did not change the amount of $\mathrm{MCH}$ conversion. The experimental conversions obtained for both MCP and $\mathrm{MCH}$ are very similar to the calculated conversions based on the results of the experiments using the pure compounds. The product yields of the 2carbon cyclopentanes obtained from the 50/50 MCP-MCH feedstock were also comparable to the yields s'jtained from the pure MCH experiments (Figure 32).

Under conditions where the active sites of a catalytic material are saturated with reactant, a maximum conversion of molecules/site/unit time is attained. This can be shown by considering Langmuir-Hinshelwood kinetics where:

$$
d \mathbf{N} / \mathrm{dt}=\mathrm{k} \theta
$$

where $\theta$ is the surface coverage of the reactant, $k$ is the reaction rate constant, $N$ is the number of molecules converted during the time, $t$. For the case of adsorptiondesorption equilibrium

$$
\begin{aligned}
& \text { rate of adsorption }=\text { rate of desorption, } \\
& \qquad \begin{array}{l}
\mathbf{K}_{\mathrm{a}}(1-\theta) \mathrm{P}_{A}=\mathrm{K}_{d} \theta \\
\theta=\mathrm{KP}_{A} /\left(1+\mathrm{KP}_{A}\right)
\end{array}
\end{aligned}
$$$$
\text { and }
$$

where $k_{a}$ and $k_{d}$ are the adsorption and desorption rate constants, respectively; $P_{A}$ is the pressure (or concentration) of the reactant; and $\mathrm{K}$ is the ratio of the adsorption and desorption rate constants. In the case considered here, the surface is saturated; 
this means that $\mathrm{KP}_{\mathrm{A}}>>1$. Thus, $\theta=1$ and the reaction rate is a constant. Increasing the amount of reactant as was done to generate the data shown in Figure 29 will not increase either $\theta$ or the reaction rate.

Another way to view this situation is the following:

$$
(d N / d t)_{0 \text { contalyot }}=\text { rate/site } \times(\text { sites } / g) \text {. }
$$

If it is assumed that each site has equal catalytic activity and that each site is completely assessable to the reactant molecules, the rate/site is a constant. Thus, the number of molecules converted per unit of catalyst per unit of time should be a constant, and independent of the feed to catalyst ratio unless the number of sites $/ \mathrm{g}$ changes. Furthermore, unless new sites are created, deactivation will eliminate sites during the reaction period so that the reaction rate should decrease with reaction time if it does not remain constant. This expectation was not what the data in Figure 30 show; rather the data in Figure 30 indicate that the number of molecules converted per unit time per catalytic site increases linearly with an increase in the feed/catalyst ratio.

Yen et al. [7] made a series of activity measurements with a catalyst whose composition and preparation were similar to the one used in our study; in fact, we prepared our catalyst following a procedure outlined in reference 7 . They utilized the catalyst in a batch reactor for the conversion of $n$-hexadecane in which the feed/catalyst was varied over a range of 2 to 12 . In the studies for $\mathbf{n}$-hexadecane conversion, the amount of catalyst was held constant and the amount of reactant in the batch reactor was varied. They reported the wt.\% conversion of $\underline{n}$-hexadecane and wt.\% sulfur (after activation at $650^{\circ} \mathrm{C}$ in air for 3 hours). We have used these data 
to calculate the relative number of molecules converted per number of molecules of $\mathrm{S}$. When viewed on this basis, the number of molecules of hexadecane converted per mole of $S$ increases with increasing feed/catalyst ratio (Figure 33). Both their data and on data obtained for MCH shown in Figure 33 show that the number of molecules of the feed increase linearly with increasing feed/catalyst ratio.

Our data indicate that there is a limiting conversion of methylcyclohexane for a particular feed/catalyst ratio. Furthermore, the extent of filling the reactor does not impact the limit of conversion for a particular feed/catalyst ratio.

The linear dependence of the number of molecules converted per unit of catalyst upon the volume of the reactant can be explained if the catalyst is responsible for generating a reactive intermediate that desorbs to the liquid or gas phase where it initiates reactions similar to that of a chain reaction. One way the number of molecules converted can depend directly upon the volume of the reactant is for the removal of the desorbed reactive intermediate (equivalent to termination in a chain reaction) to depend directly upon the volume of the feed.

For a mechanism involving a desorbed reactive intermediate, we have the following:

\section{Number of species generated/unit of catalyst/time $=$ constant ( $\mathbf{N}$ intermediates),


Furthermore,
$N_{\text {Imernodbates }} \propto 1 /$ termination,
termination $\propto 1 /$ volume of reactsnt,
and,
$N_{\text {Imermediates }} \propto$ volume of reactant.

Thus, the reaction rate becomes

rate/unit of catalyst $\propto$ constant $x$ volume of reactant.

It is realized that a similar dependence of the extent of reaction upon the reactant/catalyst ratio can be obtained if instead of a reactive intermediate that desorbs from the catalyst a component of the catalyst would dissolve into the reactant and initiate a reaction. However, the dissolved species would have to become poisoned after about six turnovers for the reactant/catalyst ratio of 2 in order to terminate the reaction at $\mathbf{2 0 \%}$ conversion (as in Figure 27). In addition, the poisoning of the dissolved species would have to depend upon the reciprocal of the reactant volume. The dissolved species is as completely surrounded by reactant even for the reactant/catalyst ratio of 2 as for higher feed/catalyst ratio, and further dilution in reactant should not decrease the possibility of poisoning. Thus, a dissolved catalyst species does not appeer reasonable; however, data do not exist at present to completely rule out this possibility.

In summary, it appears that very similar conversion data, and its dependence upon the reactant/catalyst ratio, have been obtained independently in two laboratories using quite different reactants but similar catalysts and reactors. The data are in agreement with a mechanism involving the formation of a reactive intermediate on the catalyst surface followed by desorption to the liquid (or gas) phase. In the liquid 
phase the reactive intermediate initiates a chain-type reaction whose chain length depends upon the concentration of the reactive intermediate in a the reactant. The postulate that the termination of the reaction varies with the reciprocal of the reactant volume leads to the direct dependence of the cumulative conversion for unit catalyst upon the reactant/catalyst ratio.

The data with the $\mathrm{Pt}_{-} \mathrm{SO}_{4}$-zirconia would not be compatible with catalysis by a superacid unless the desorbed species was a carbonium ion type intermediate. It appears highly unlikely to us that a carbonium ion should dissolve in the methylcyclohexane or hexadecane; hence, a neutral species, sith as a free radical, would appear more likely as a reactive intermediate.

The above discussion is limired to the case of the sulfated zirconia catalyst. In fact, preliminary data with catalysts prepared following the recipes in references 32 and 33 do not give this type of conversion data. The multi-metallic catalyst containing both iron and manganese as well as sulfate do not appear to exhibit the trend shown in Figures 29, 30, and 33. Furthermore, preliminary data with the $\mathrm{Pt} / \mathrm{SO}_{4} / \mathrm{ZrO}_{2}$ catalyst in a plug flow reactor under conditions where the reactant is in the gas phase indicate that the turnover/sulfur is much larger than 6.

Sulfated $\mathrm{Fe} / \mathrm{Mn} / \mathrm{ZrO}_{2}$ Catalysts

A number of $\mathrm{Fe} / \mathrm{Mn} / \mathrm{ZrO}_{2} / \mathrm{SO}_{4}$ catalysts were prepared for study. In addition to the catalysts prepared in-house, a proprietary catalyst was received from an industrial source (catalyst A). The analyses of the as-received catalysts are given in Table 6. The catalysts were activated prior to activity testing using a $725^{\circ} \mathrm{C}$ temperature for 2 hours in air. The sulfur contents of the activated catalysts are given in Table 7. 
The activated catalysts were tested in the batch microautoclaves using a temperature of $150^{\circ} \mathrm{C}$, a residence time of 2 hours, 1 atmosphere of $H_{2}$, and a feed/catalyst ratio of 2. MCH and cetane were used as feedstocks for the activity testing. The conversions obtained are shown in Figure 34. As can be seen in this figure, two of the catalyst formulations (SR003 and SR011) were relatively inactive for the conversion of $\mathrm{MCH}$ and cetane. The other three formulations (catalyst A, SRO07

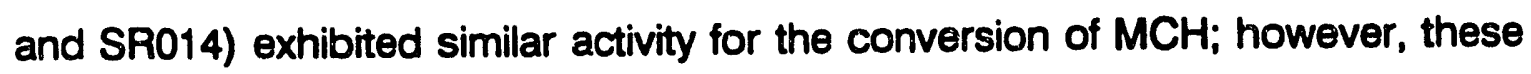
catalysts showed very different conversions of cetane. Catalyst A and SR014 produced relatively low cetane conversions. The SR007 formulation produced the highest cetane conversion (34.34 wt.\%).

The characterization data of these catalysts (Tables 6-8) do not provide a clear reason(s) for the observed differences in activity. The low MCH conversions obtained using the SROO3 and SR011 catalysts may be due to the different $\mathrm{Fe}$ and Mn loadings. These data suggest there may be an optimum Fe and Mn concentration. The sulfur content of the catalysts after activation (Table 7) or after reaction (Table 8) does not appear to correlate with the $\mathrm{MCH}$ or the cetane conversions.

The major products obtained from the conversion of $\mathrm{MCH}$ using these catalysts were 2-carbon cyclopentanes as was observed using the chlorided $\mathrm{Pt} / \mathrm{Al}_{2} \mathrm{O}_{3}$ catalysts. Since it was observed that increasing the $\mathrm{H}_{2}$ pressure changed the selectivity (ringopening) of the chlorided $\mathrm{Pt} / \mathrm{Al}_{2} \mathrm{O}_{3}$ catalysts, a series of experiments using different pressures and the SR014 catalyst was completed. The conversions and selectivities obtained from these experiments are shown in Figure 35. It appears that increasing the pressure from 0 to $750 \mathrm{psig}$ had no effect on the conversion or selectivity. 
However, at reactor pressures grcater than 700 psig, the conversion of $\mathrm{MCH}$ is significantly reduced with no change in selectivity. Initially it was proposed that the loss in activity at the higher pressures was due to the loss of sulfur from the catalyst.

The catalyst after the reaction with $\mathrm{MCH}$ were dried in a vacuum oven to remove any hydrocarbons and submitted for $S$ analysis. The results shown in Table 9 indicate that the lower activity obtained at higher pressures was not due to the loss of sulfur from the catalyst.

\section{Sulfated $\mathrm{Ni}$ and $\mathrm{Rh} /$ Zirconium Catalysts}

A series of sulfated zirconium catalysts were prepared using Rh (SR022A) and $\mathrm{Ni}$ (SR022B) in place of Pt. The catalysts were activated at $725^{\circ} \mathrm{C}$ for 2 hours in air prior to activity testing. The catalysts were run in the batch autoclave reactors using a temperature of $150^{\circ} \mathrm{C}$, a 15 minute residence time, 1 atmosphere of hydrogen and a $\mathrm{MCH}$ :catalyst ratio of 2. The analysis and activity of these catalysts are compared to a sulfated $\mathrm{Pt} / \mathrm{ZrO}_{2}$ catalyst previously described in Table 10.

The results shown in Twble 9 indicate that the conversion of the catalyst impregnated with $\mathrm{Rh}$ was slightly higher than that obtained with the sulfated $\mathrm{Pt} / \mathrm{ZrO}_{2}$ catalyst. The nickel catalyst showed very little activity. The major products obtained using all three catalysts were 2-carbon cyclopentanes. 


\section{REFERENCES}

1. E. F. Schvarzenback, Proc. Am. Petrol. Inst., 37, Section III, 354 (1957).

2. D. H. Belden and V. Haensel, Proc. Am. Petrol. Ind., 37, Section III, 354 (1957).

3. T. Y. Yan, T. J. Huang, W. O. Haag, U.S. Patent 4,049,539, September, 1977.

4. A. G. Goble, P. A. Lawrance, Proc. 3rd Int. Cong. Catalysis, Amsterdam, Vol. 1, 320, 1964.

5. F. Garin, D. Andriamasinoro, a. Abdulsamad and J. Sommer, J. Catal., 131, $1991,199$.

6. T. Hosoi, T. Shimidzu, S. Itoh, S. Baba and H. Takaoka, Am. Chem. Soc., Div. Petrol. Chem., 33, 1988, 562.

7. M. Y. Wen, I. Wender, and J. W. Tierney, Energy \& Fuels, 4, 1990, 72.

8. F. R. Chen, G. Coudurier, J. F. Joly and J. C. Vedrine, Am. Chem. Soc., Div. Petrol. Chem., 36, 1991, 878.

9. K. Mukaida, T. Miyoshi and T. Satoh, in "Acid-Base Catalysis", (K. Tanabe, H. Hattori, T. Yamaguchi and T. Tanaka, eds.), Kodansha, Tokyo, 1988, pp 363369.

10. C. Morterra and L. Marchese, in "Acid-Base Catalysis", (K. Tanabe, H. Hattori, T. Yamaguchi and T. Tanaka, eds.), Kodansha, Tokyo, 1988, pp 197-205.

11. T. Hochmann and K. Setínek, Collect. Czech Chem. Commun., 56, 1991, 1404, 1417.

12. K. Ebitani, J. Konishi and H. Hattori, J. Catal., 130, 1991, 257.

13. K. Ebitani, J. Konishi, A. Horie, H. Hattori and K. Tanabe, "Acid-Base Catalysis", (K. Tanabe, H. Hattori, T. Yamaguchi and T. Tanaka, eds.), Kodansha, Tokyo, 1988, pp 491-496.

14. M. Hino, S. Kobayashi and K. Arata, J. Am. Chem. Soc., 101, 1979, 6439.

15. O. Takahashi, T. Yamaughi, T. Sakuhara, H. Hattori and K. Tanabe, Bull. Chem. Soc., Japan, 53, 1980, 1807.

16. T. Ishida, T. Yamaguchi and K. Tanabe, Chem. Lett, 1988, 1869.

17. P. S. Kumbhar and G. D. Yarav, Chem. Eng. Sci., 44, 1989, 2535.

18. T. Yamaguchi, Appl. Catal., 61, 1990, 1.

19. M. S. Scurrell, Appl. Catal., 34, 1987, 109.

20. K. Arata, M. Hino and N. Yamagata, Bull. Chem. Soc. Japan, 63, 1990, 244.

21. K. Ebitani, J. Tsuji, H. Hattori and H. Kita, J. Catal., 135, 1992, 609.

22. K. Tanabe, Mater. Chem. and Phys., 13, 1985, 347.

23. M. Ozawa and M. Kimura, J. Less-Common Metals, 171, 1991, 195.

24. K. Ebitani, H. Konno, T. Tanaka and H. Hattori, J. Catal., 135, 1992, 60.

25. K. Tanabe and T. Yamaguchi in "Successful Design of Catalysts", (T. Inui, ed.), Elsevier Sci. Pub., Amsterdam, 1988, pp 99-110.

26. K. Tanabe, "Critical Revues in Surfaces Chemistry", 1, 1990, 1.

27. M. Bensitel, O. Saur and J. C. Lavalley, Mater. Chem. and Phys., 17, 1987, 249.

28. T. Yamaguchi and K. Tanabe, Mater. Chem. and Phys., 16, 1986, 67.

29. K. Arata, Mater. Chem. and Phys., 26, 1990, 213.

30. B. Umansky, J. Engelhardt and W. K. Hall, J. Catal., 127, 1991, 128.

31. U.S. Patent, 3,132,110, May 5, 1964.

32. U.S. Patent, 5,019,671, May 28, 1991.

33. U.S. Patent, 4,918,041, April 17, 1990. 


\section{Table 1}

Analysis of Feedstocks

$\begin{array}{cccc}\text { C } & \begin{array}{c}\text { Black Thunder } \\ \text { Naphtha }\end{array} & & \begin{array}{c}\text { Illinois \#6 } \\ \text { Naphtha }\end{array} \\ \text { H } & 85.23 \% & & 85.57 \% \\ \text { N } & 12.23 \% & & 13.24 \% \\ \text { S } & 3510 \mathrm{ppm} & & 795 \mathrm{ppm}\end{array}$

Table 2

Analysis of Catalysts

Co-Mo-Alumina

$2.7 w t . \%$ Co

11.1 wt. \% Mo

$288 \mathrm{~m}^{2} / \mathrm{g}$
Ni-Mo-Alumina

$3.1 \% \mathrm{Ni}$

$13.3 \% \mathrm{MO}$

$180 \mathrm{~m}^{2} / \mathrm{g}$ 
TABLE 3

Process Summary

Black Thunder

Hydrotreatment

Pass \#

(Run Hours

$1(0-165)$

$2(0-143)$

$3(0-127)$

$(127-148)$

$2(149-284)$

3 (185-408)
Catalyst

CoMo

CoMo

NiMo

4.5
WHSV

\# feed/\# catalyst/hr

$.9,1.0$

$.9,1.0$

.8
Temp Pressure

( $\left.{ }^{\circ} \mathrm{C}\right) \quad$ (psig)

$418 \quad 2010$

$418 \quad 2010$

$403 \quad 2000$
Illinois \#6

Hydrotreatment

$\begin{array}{llllll}1(127-148) & \text { NiMo } & 4.5 & .8 & 352 & 2000 \\ 2(149-284) & \text { NiMo } & 1.1 & .2 & 352 & 2000 \\ 3(185-408) & \text { NiMo } & 1.8 & .3 & 403 & 2000\end{array}$


Table 4

Naphtha Compositions

Compound

propane

iso-butane

n-butane

iso-pentane

n-pentane

2,2-dimethylbutane

2,3-dimethylbutane

cyclopentane

3-methylpentane

n-hexane

methylcyclopentane

3,3-dimethylpentane

cyclohexane

2-methylhexane

2,3-dimethylpentane

1,1-dimethylcyclopentane

3-methylhexane

1,cis-3-dimethylcyclopentane

1,trans-3-dimethylcyclopentane

1,trans-2-dimethylcyclopentane

n-heptane

methylcyclohexane

ethylcyclopentane

toluene (coelution)

3-methylheptane

1,trans-4-dimethylcyclohexane

1,cis-3-dimethylcyclohexane

1-methyl-3-ethylcyclopentane

1-methyl-2-ethylcyclopentane

Table 1 Continued
III. \#6

Hydrotreated

Naphtha

(Wt. \%)

.04

.06

.86

.59

1.31

.004

.04

1.11

.41

1.53

1.74

.01

8.12

.16

1.05

.10

.79

.30

.36

.49

4.63

10.87

.97

1.95

.65

1.52

.73

.25

.18 \begin{tabular}{l} 
Black Thunder \\
Hydrotreated \\
Naphtha \\
(Wt. \%) \\
\hline
\end{tabular}

.07

.05

.33

.30

.63

.01

.03

.69

.29

.78

1.11

.01

4.91

.43

.28

.06

.42

.20

.24

.43

.66

5.03

.54

3.59

.44

.72

.31

.16

.13 
Compound

1-methyl-1-ethylcyclopentane

2-C cyclohexane

n-octane (coelution)

1,cis-2-dimethylcyclohexane

ethylcyclohexane

3-C cyclohexane

ethylbenzene

$m$ \& p-xylene

3-methyloctane

1-methyl, trans-2-cis-

3-trimethylcyclohexane

1-methyl, trans-2, cis-

4-trimethylcyclohexane

$0-x y l e n e$ (coelution)

4-C cyclopentane

1-methyl-4-ethylcyclohexane

1-methyl-3-ethylcyclohexane

1-methyl-2-ethylcyclohexane

1-methylethylbenzene

i-propylcyclohexane

butyl cyclohexane

n-propylbenzene

1-methyl, 2-ethylbenzene

3-C benzene

3-C benzene

1-methyl-4-(1-methylethyl) cyclohexane

$\mathrm{C}_{10} \mathrm{H}_{20} / \mathrm{C}_{9} \mathrm{H}_{16}$ (hexahydroindan)

3-C benzene

Indol/4-C cyclohexane

Indan

4-C-cyclohexene

1-methyl-3-propylbenzene

5-methylindan

1-C indan/biphenyl
III. $\# 6$

Hydrotreated

Naphtha

(Wt. \%)

.39

.71

1.51

.31

4.75

.06

.82

1.20

.36

.99

.31

.55

.40

.68

.15

.33

.03

2.58

.36

.59

.57

.15

.17

.51

1.28

.64

1.17

.68

.52

.25

.37

1.06
.62

Black Thunder Hydrotreated Naphtha (Wt. \%)

.31

.21

.65

.16

2.94

.21

2.15

.98

.39

.20

.73

.23

.39

.12

.29

.04

1.56

.20

.26

.78

.27

.15

.44

.71

.13

.26

.70

.64

.30

2.33 


\section{Compound}

undecane

acenaphthene/biphenyl

2-C indan or $1-C$ tetralin

1-C indan

5-C benzene

5- $C$ benzene

1-C indan

2-C indan/1-C tetralin

2-C indan/1-C tetralin

2-C indan/1-C tetralin

2-C indan/1-C tetralin dodecane

2-C indan/1-C tetralin

2-C indan $/ 1-C$ tetralin

2-C indan/1-C tetralin

$2-C$ indan $/ 1-C$ tetralin

2-C indan/1-C tetralin

6-methyltetralin
III. \#6

Hydrotreated

Naphtha

(Wt. \%)

.23

.36

1.06

1.18

.38

.09

1.56

.70

.23

.34

.51

.35

.47

.27

.21

.31

.31

1.60
Black Thunder Hydrotreated Naphtha (Wt. \%)

.16

.21

1.49

1.61

.10

.15

1.87

1.25

.56

.80

.89

.89

.03

.18

.04

.13

.30

1.30 
Table 5

Surface Compositions of the 4 and 10 wt.\%

Pt-alumina Catalysts Following Various Treatments

Element, Atomic \%

\begin{tabular}{|c|c|c|c|c|c|}
\hline Catalyst & Pretreatment & $\underline{P t}$ & $\underline{A l}$ & $\underline{0}$ & $\underline{\mathrm{Cl}}$ \\
\hline 4 wt.\% Pt & None, as prepared & 0.27 & $36.6(2.27)$ & 59.9 & 3.3 \\
\hline 4 wt.\% Pt & $\mathrm{H}_{2} / 300^{\circ} \mathrm{C} / 1 \mathrm{hr}$. & 0.22 & $42.0(2.31)$ & 58.0 & $N / D^{a}$ \\
\hline $4 w t . \%$ Pt & $\mathrm{CCl}_{4} / 300^{\circ} \mathrm{C} / 25 \mathrm{~min}$. & 0.24 & $23.1(1.84)$ & 73.6 & 3.1 \\
\hline $10 \mathrm{wt} . \% \mathrm{Pt}$ & $\mathrm{CCl}_{4} / 300^{\circ} \mathrm{C} / \sim 14 \mathrm{hr} .^{b}$ & 0.41 & $42.0(2.20)$ & 53.1 & 4.5 \\
\hline $10 w t . \% \mathrm{Pt}$ & As prepared ${ }^{\circ}$ & 1.2 & $13.6(2.43)$ & 85.2 & N/D \\
\hline 10 wt.\% Pt & $\mathrm{H}_{2} / 300^{\circ} \mathrm{C} / 1 \mathrm{hr} .{ }^{\circ}$ & 1.7 & $62.391 .94)$ & 36.1 & N/D \\
\hline $10 w t . \% \mathrm{Pt}$ & $\mathrm{CCl}_{\sqrt{ }} / 300^{\circ} \mathrm{C} / 1 \mathrm{hr}^{\mathrm{c}}$ & 2.07 & $67.8(3.25)$ & 26.6 & 3.47 \\
\hline $10 w t . \% \mathrm{Pt}$ & $\mathrm{H}_{2} / 300^{\circ} \mathrm{C} / 1 \mathrm{hr}^{c}$ & 1.60 & $66.9(2.45)$ & 30.2 & 1.29 \\
\hline
\end{tabular}

a $\quad$ N/D $=$ not determined.

b Chlorided in plug-flow reactor and transferred to XPS chamber.

c Sample treated successfully in order from top to bottom. 
Table 6

Analysis of Sulfated $\mathrm{Fe} / \mathrm{Mn} / \mathrm{ZrO}_{2}$ Catalysts (as-recieved)

Catalyst A SRO03 $\underline{\text { SR007 }}$ SR011 $\underline{\text { SR014 }}$

$\begin{array}{llllll}\text { Fe (wt.\%) } & 1.45 & 1.42 & 1.40 & 0.92 & 1.50 \\ \text { Mn (wt.\%) } & 0.32 & 0.19 & 0.30 & 2.82 & 0.47 \\ \text { S (wt.\%) } & 1.85 & 0.51 & 0.53 & 6.90 & 0.73\end{array}$

Table 7

Sulfur Content of Catalysts after Activation $\left(725^{\circ} \mathrm{C}, 2 \mathrm{hrs}\right.$, air)

Catalyst A SR003 SR007 SR011 $\underline{\text { SR014 }}$

$\begin{array}{llllll}S \text { (wt.\%) } & 1.13 & 0.46 & 0.53 & 1.84 & 0.66\end{array}$

Table 8

Analysis of Spent Sulfated $\mathrm{Fe} / \mathrm{Mn} / \mathrm{ZrO}_{2}$ Catalysts

$\begin{array}{lllll}\text { Catalyst A } & \text { SR003 } & \text { SR007 } & \text { SR011 } & \text { SR014 }\end{array}$

$\begin{array}{lccccc}\text { Carbon (wt.\%) } & 0.62 & 0.18 & 0.15 & 0.24 & 0.34 \\ \text { S (wt.\%) } & 1.30 & 0.45 & 0.58 & 2.02 & 0.65 \\ \text { Phase } & \text { Tetragonal } & \text { Tetragonal } & \text { Tetragonal } & \begin{array}{c}\text { Tetragonal + } \\ \text { Monoclinic }\end{array} & \text { Not Determined } \\ & & & & \text { (trace) }\end{array}$




\section{Table 9}

Sulfur Content of Spent Sulfated $\mathrm{Fe} / \mathrm{Mn} / \mathrm{ZrO}_{2}$ Catalyst (SR014) using Different Total Pressures

$\begin{array}{cc}\text { Pressure (psig) } & \underline{S \text { (wt.\%) }} \\ 100 & 0.66 \\ 400 & 0.69 \\ 600 & 0.68 \\ 700 & 0.66 \\ 1000 & 0.66\end{array}$


Table 10

Analysis and Activity of Sulfated Metal $\mathrm{ZrO}_{2}$ Catalysts

$\begin{array}{lcccc}\text { Catalyst } & \text { Metal (wt.\%) } & & \text { Sulfur (wt.\%) } & \begin{array}{c}M C H \\ \text { Conversion (wt.\%) }\end{array} \\ \text { SR022A } & \text { Rh (0.73) } & 3.56 & 21.6 \\ \text { SR022B } & \text { Ni (0.96) } & 3.45 & 3.4 \\ \text { SR \#4 } & \text { Pt (0.57) } & 2.56 & 17.6\end{array}$




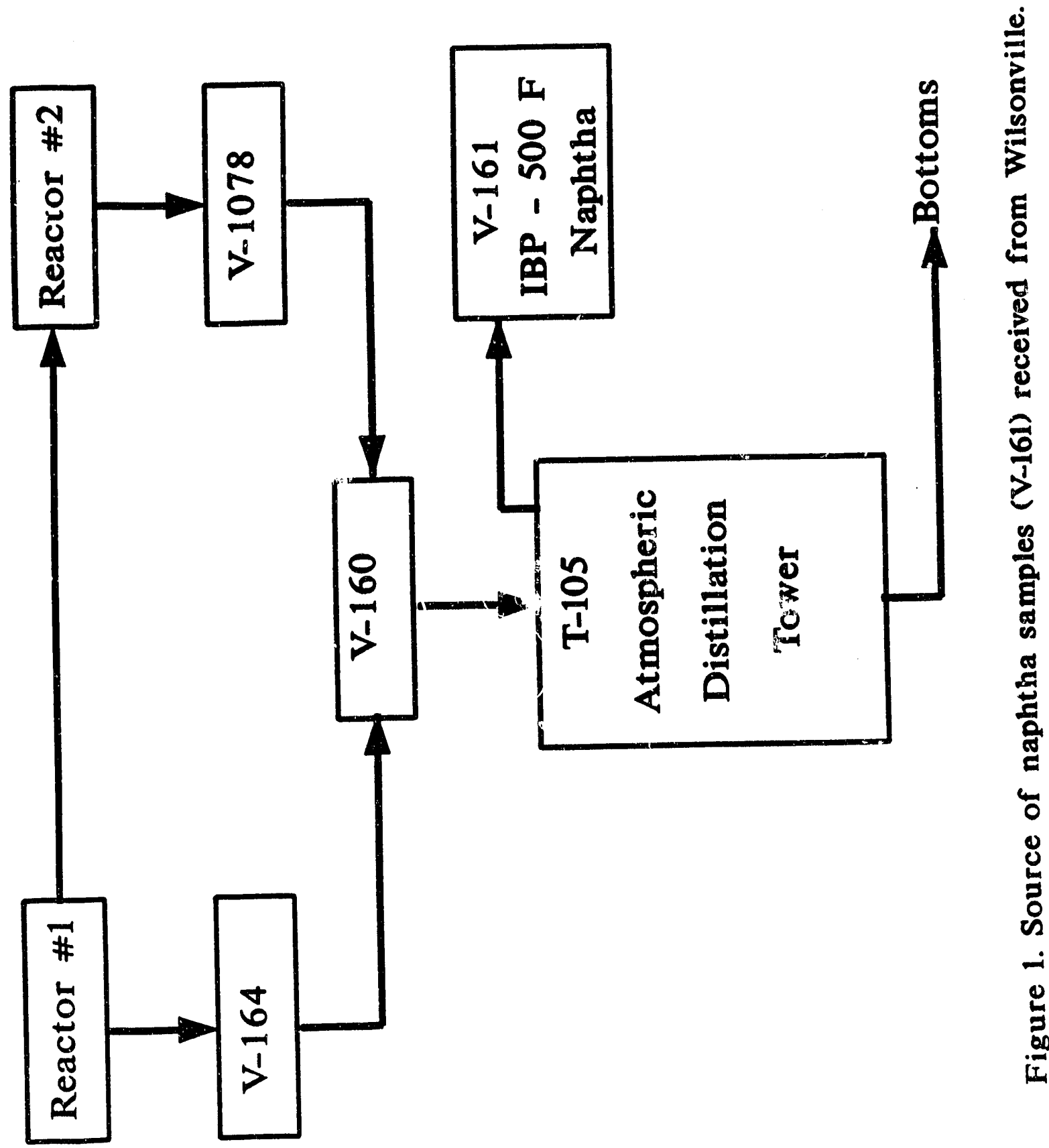




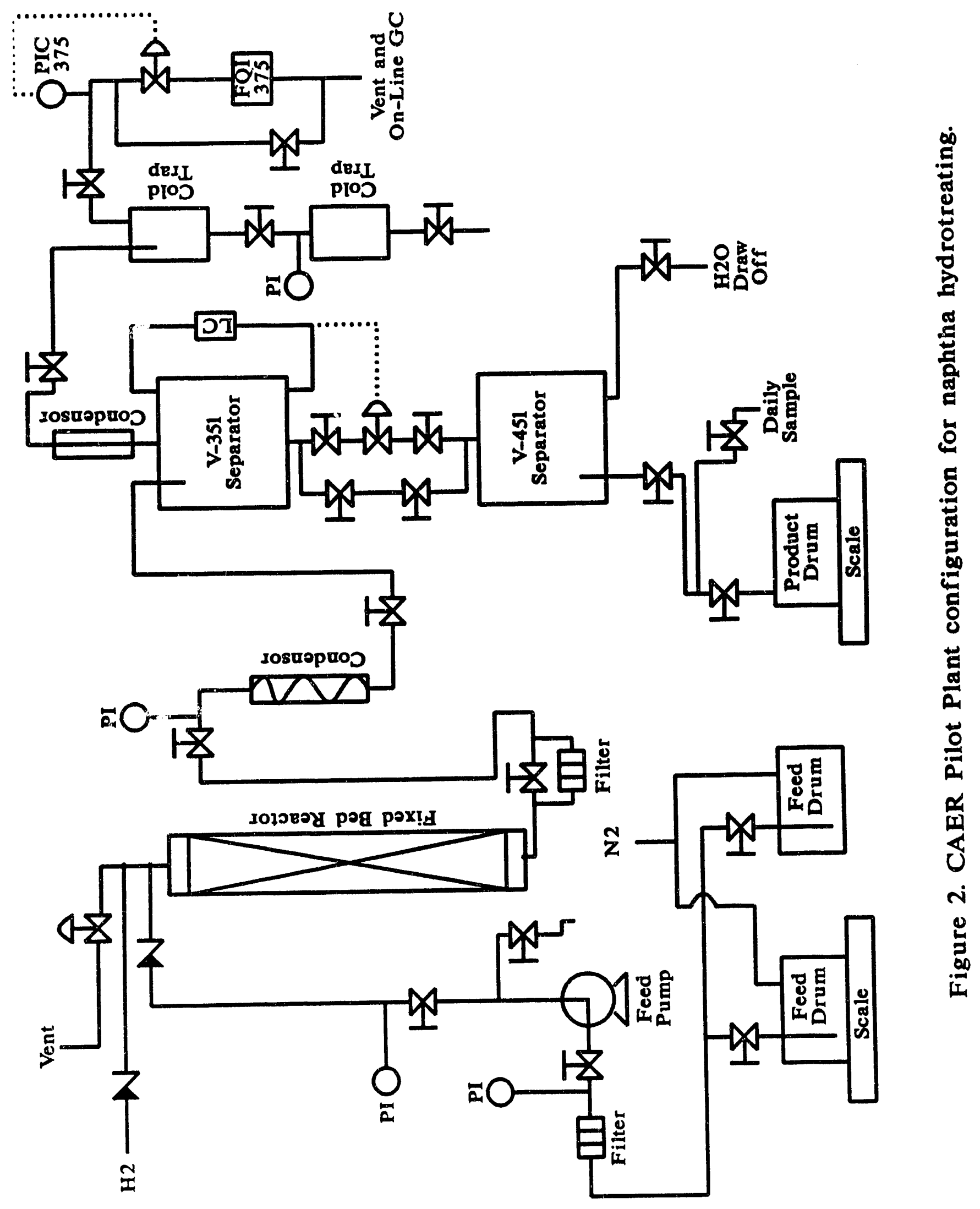




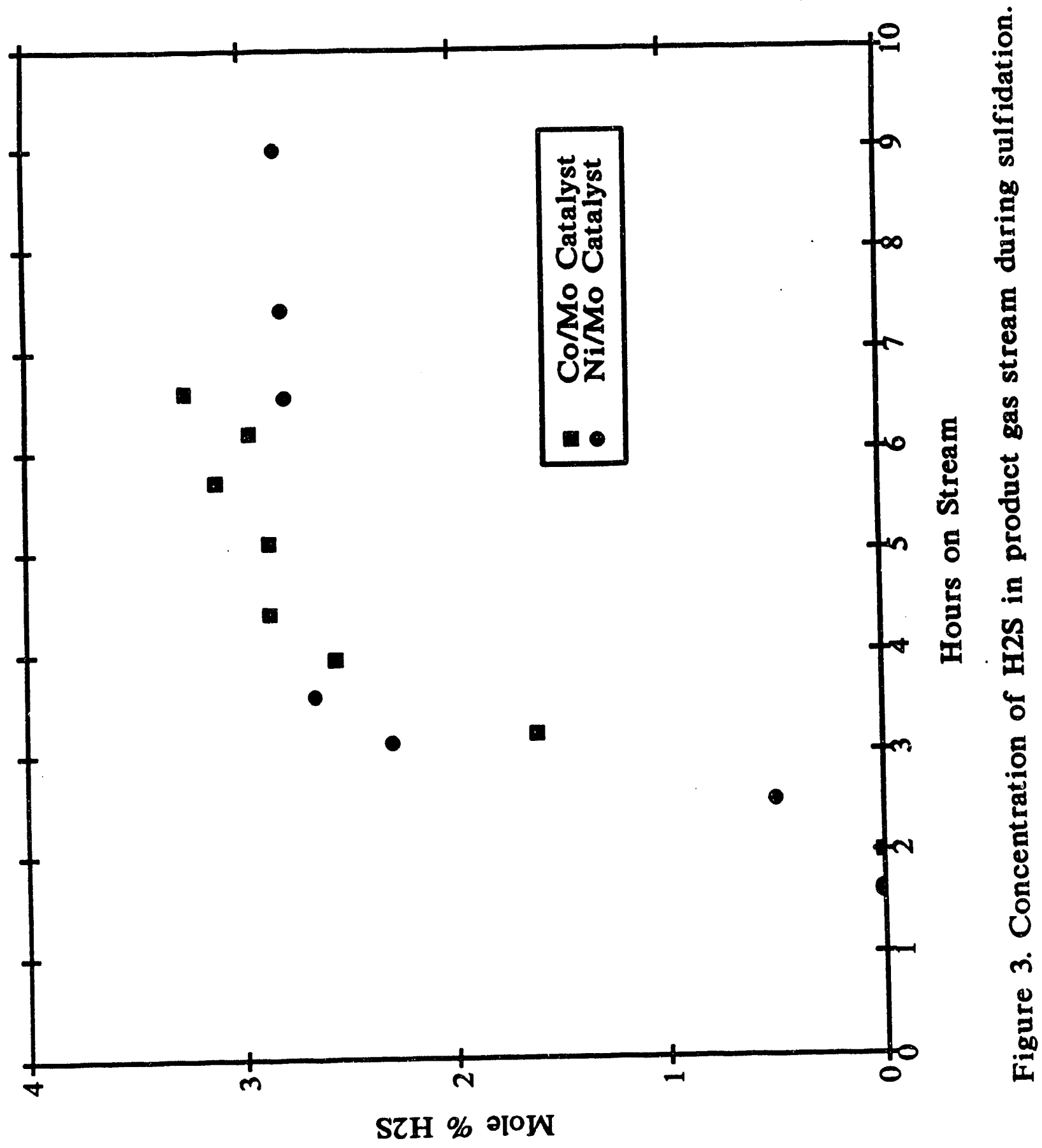




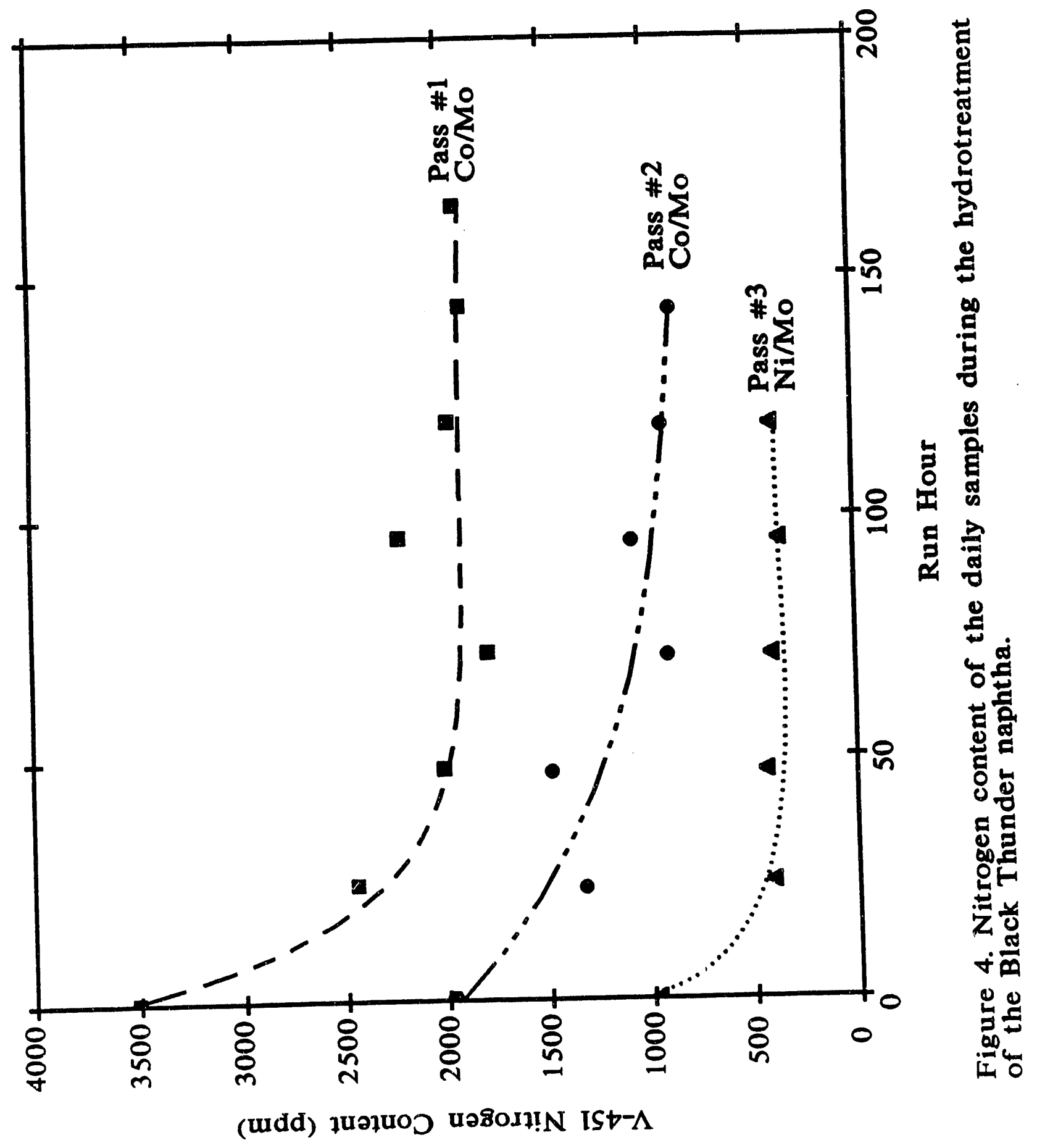




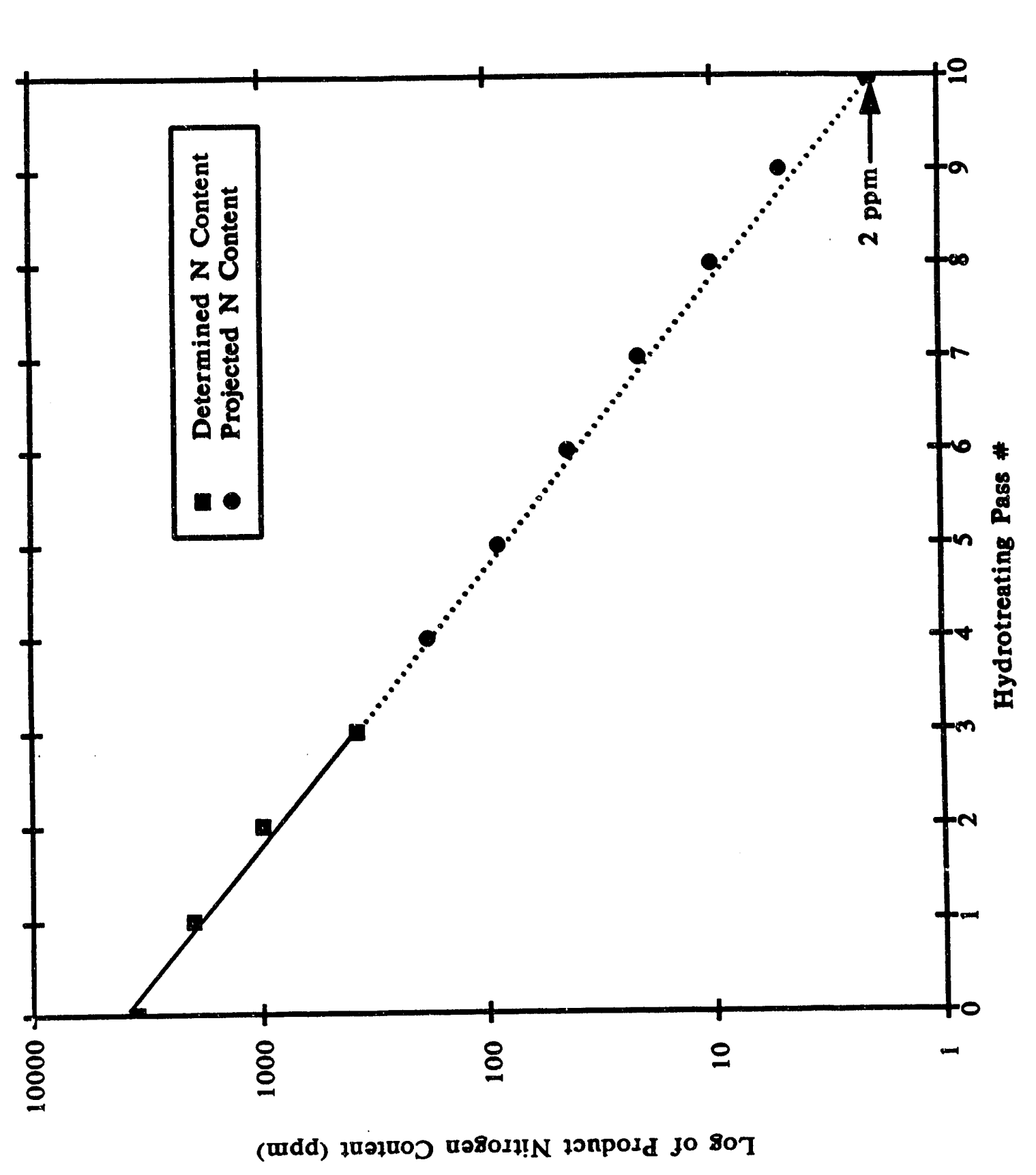

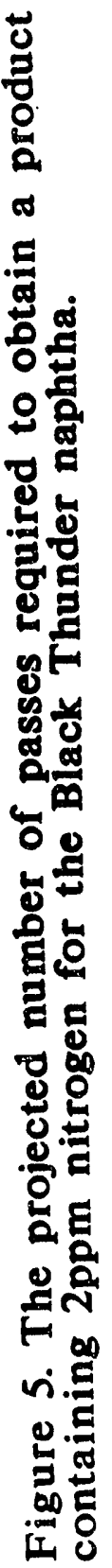




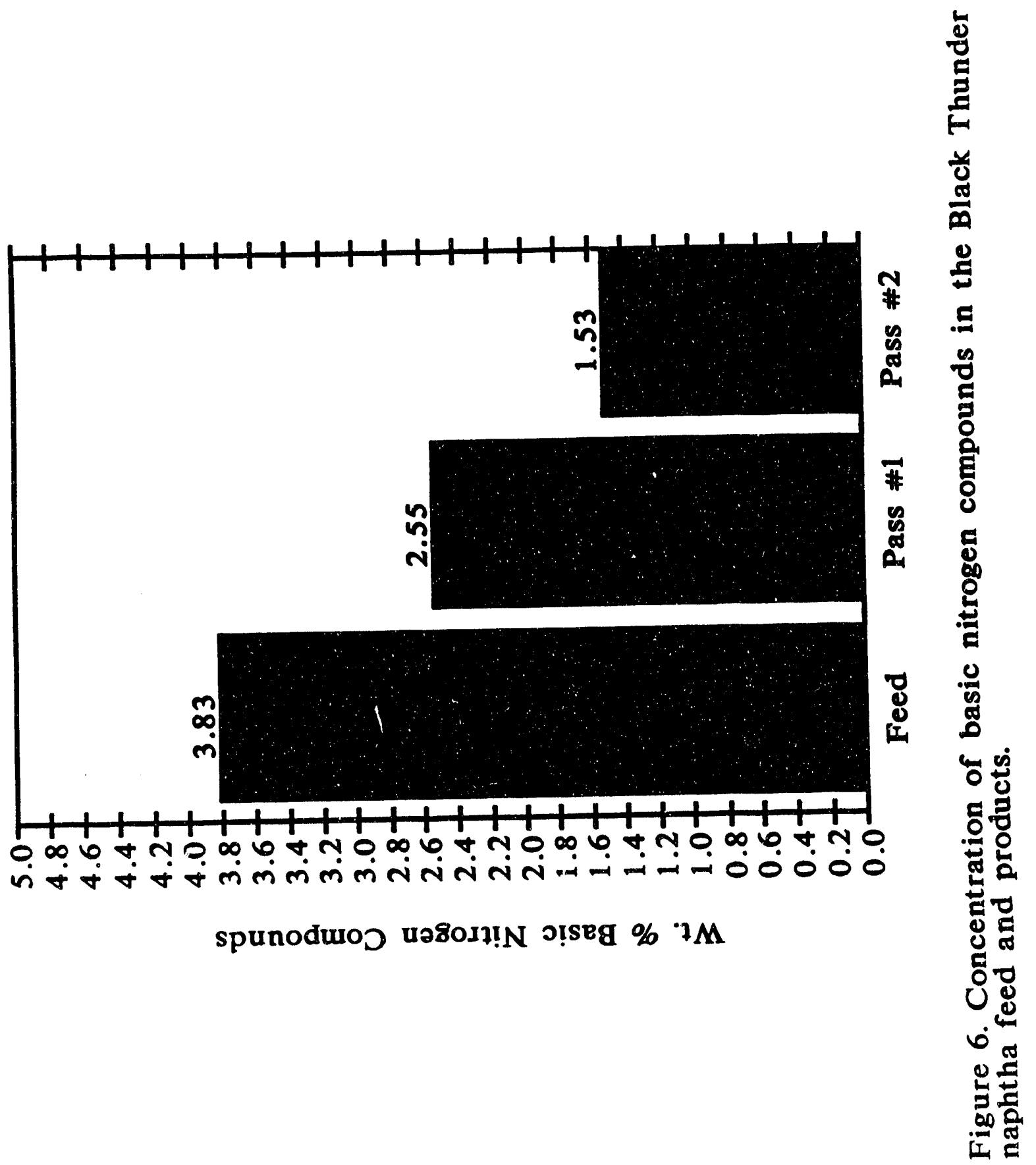




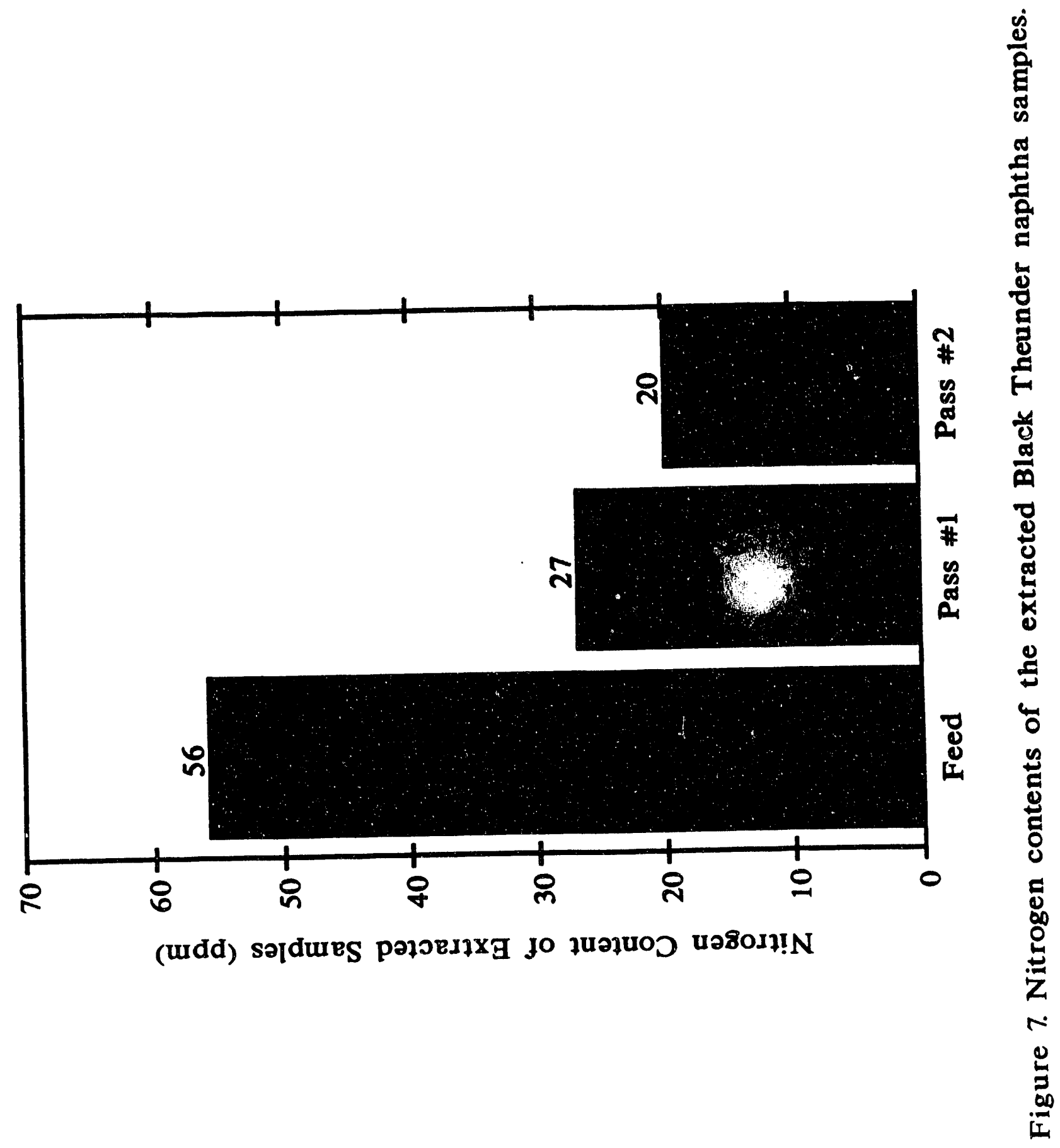




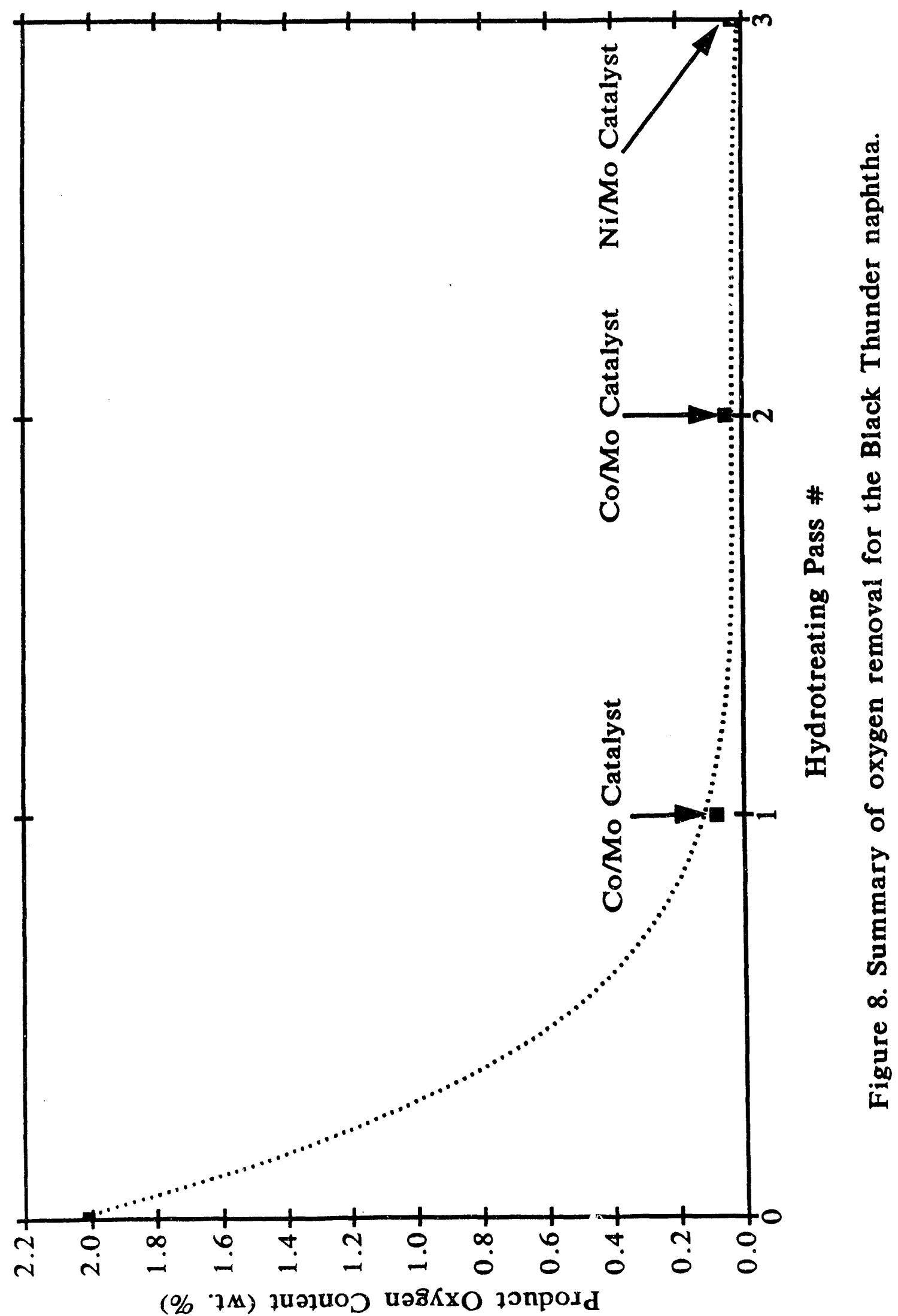




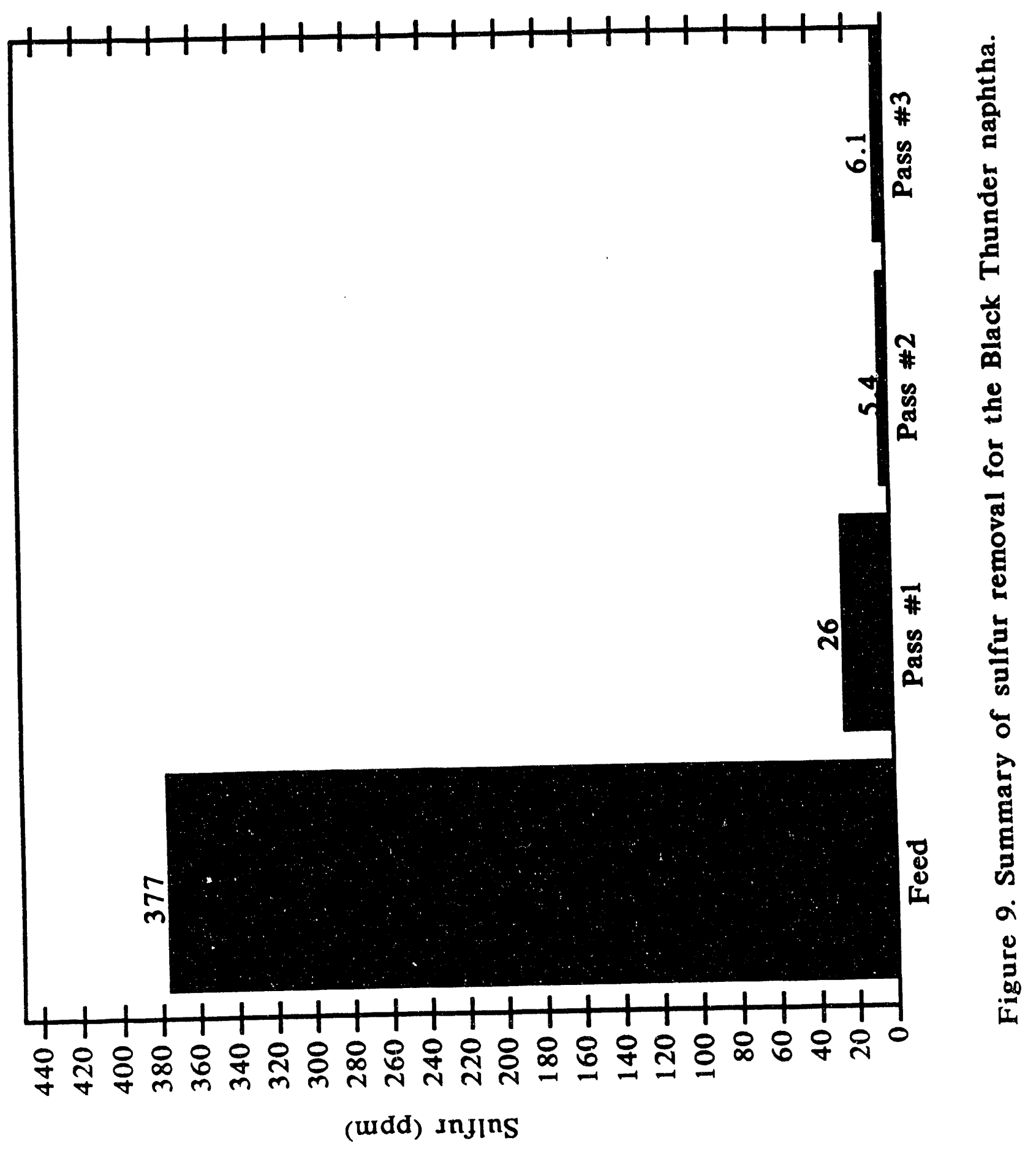




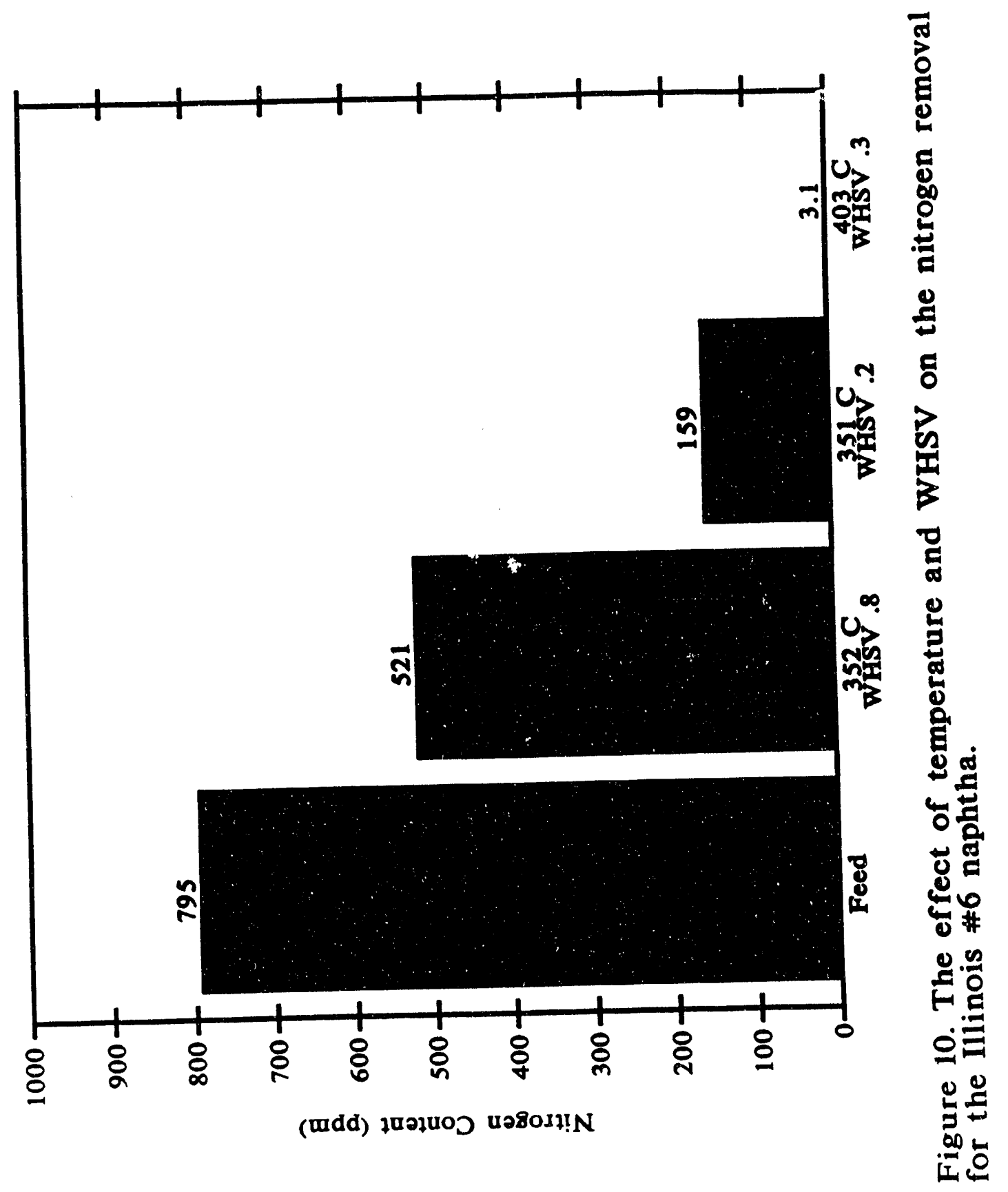




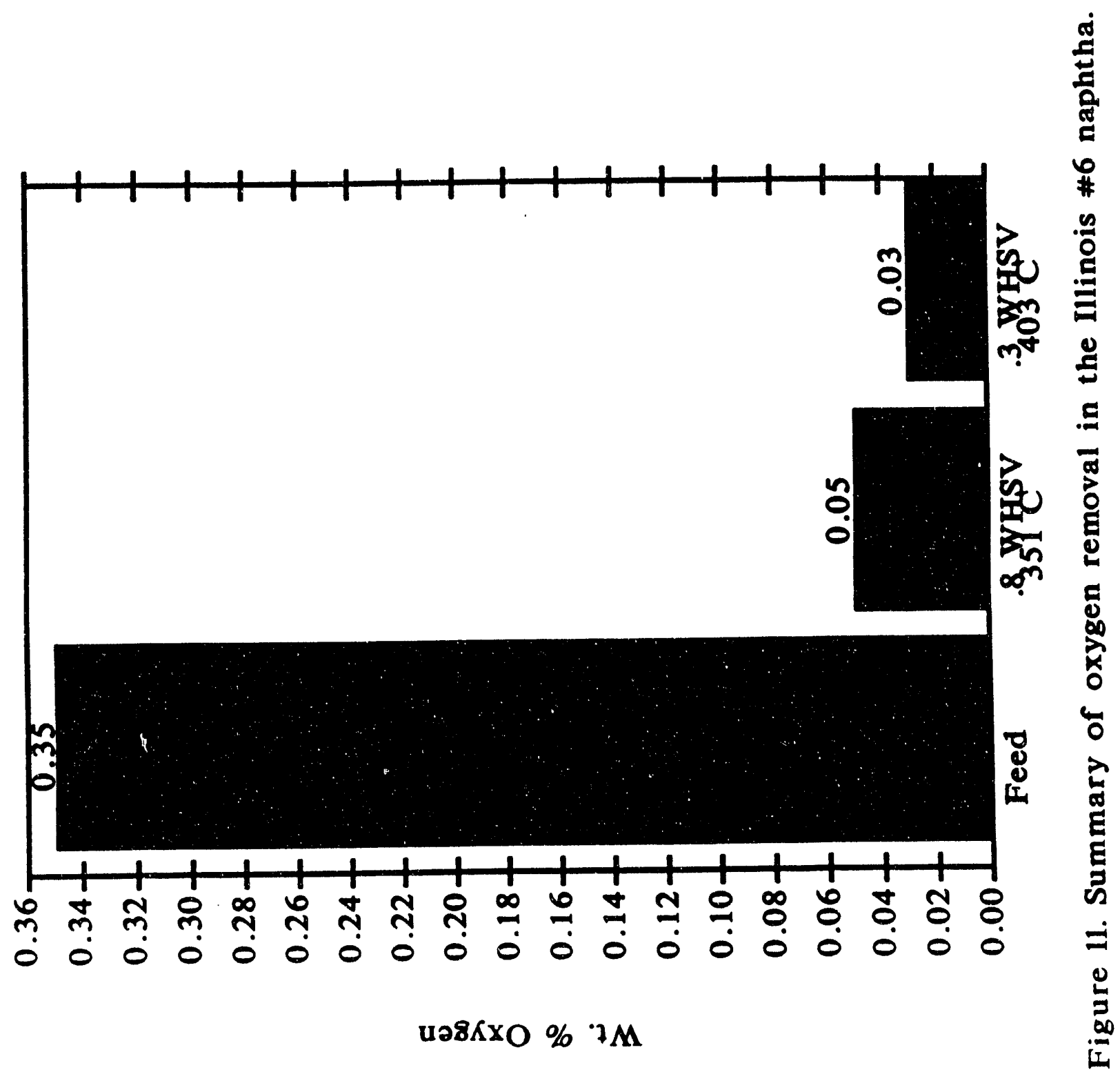




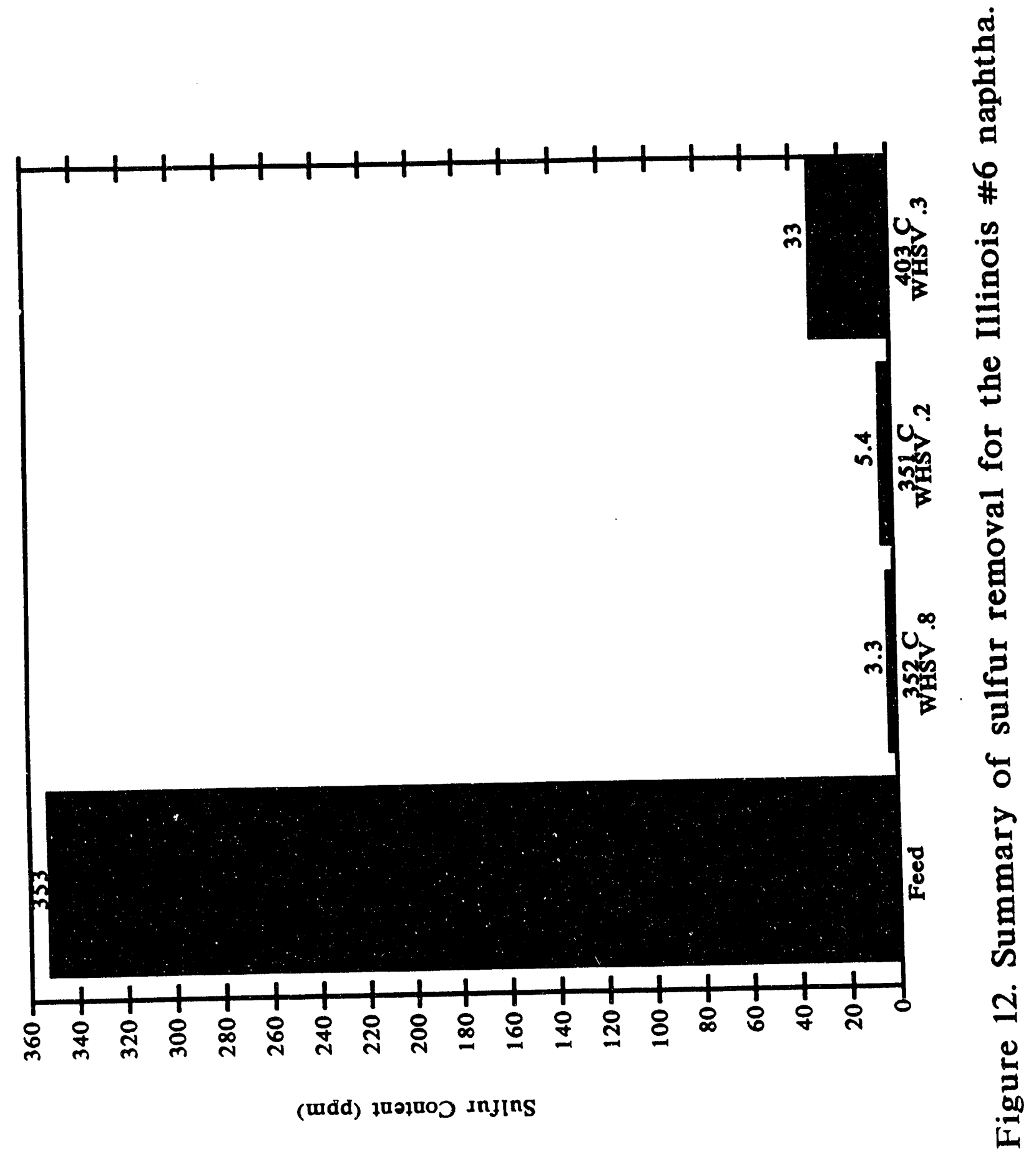




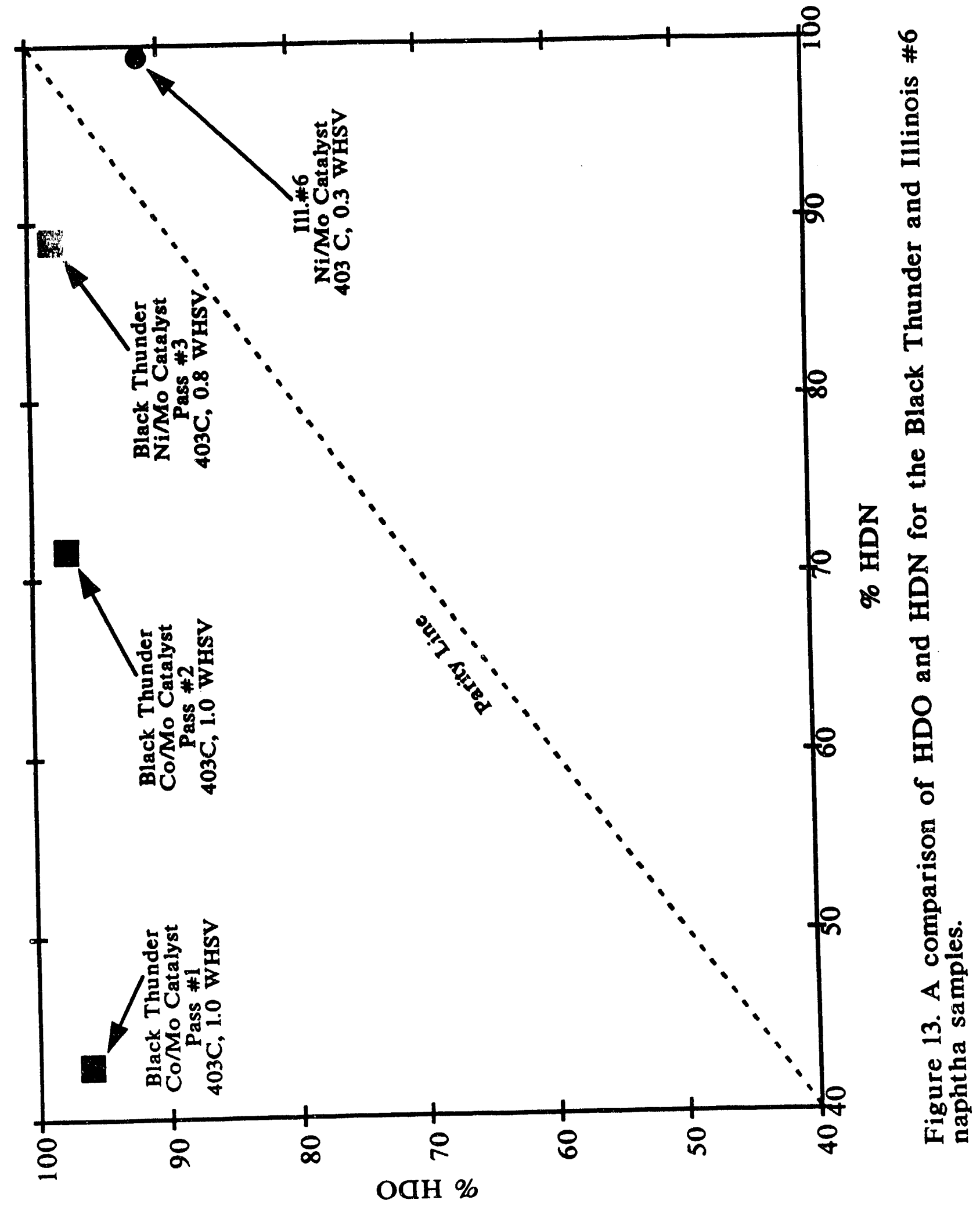




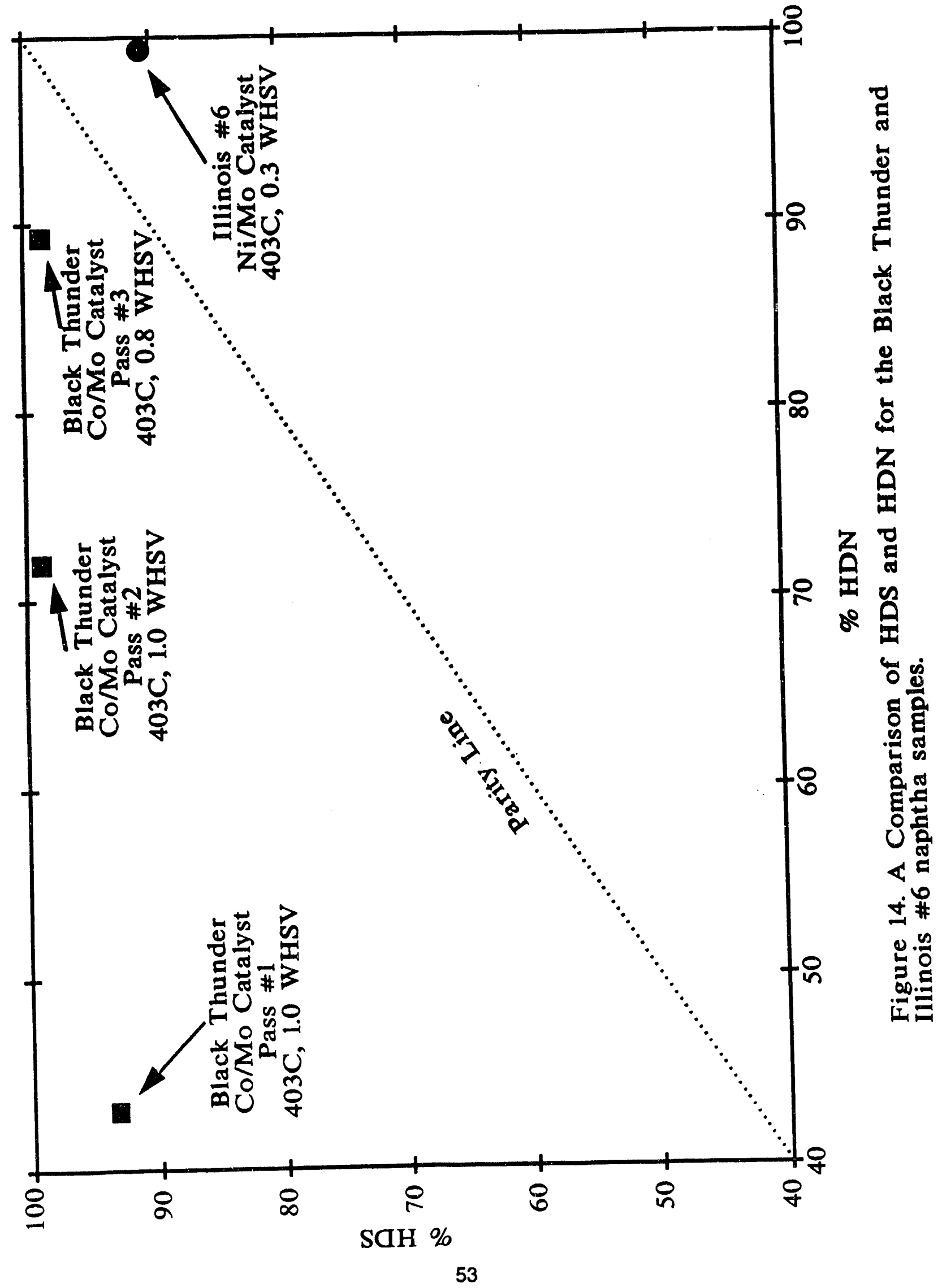




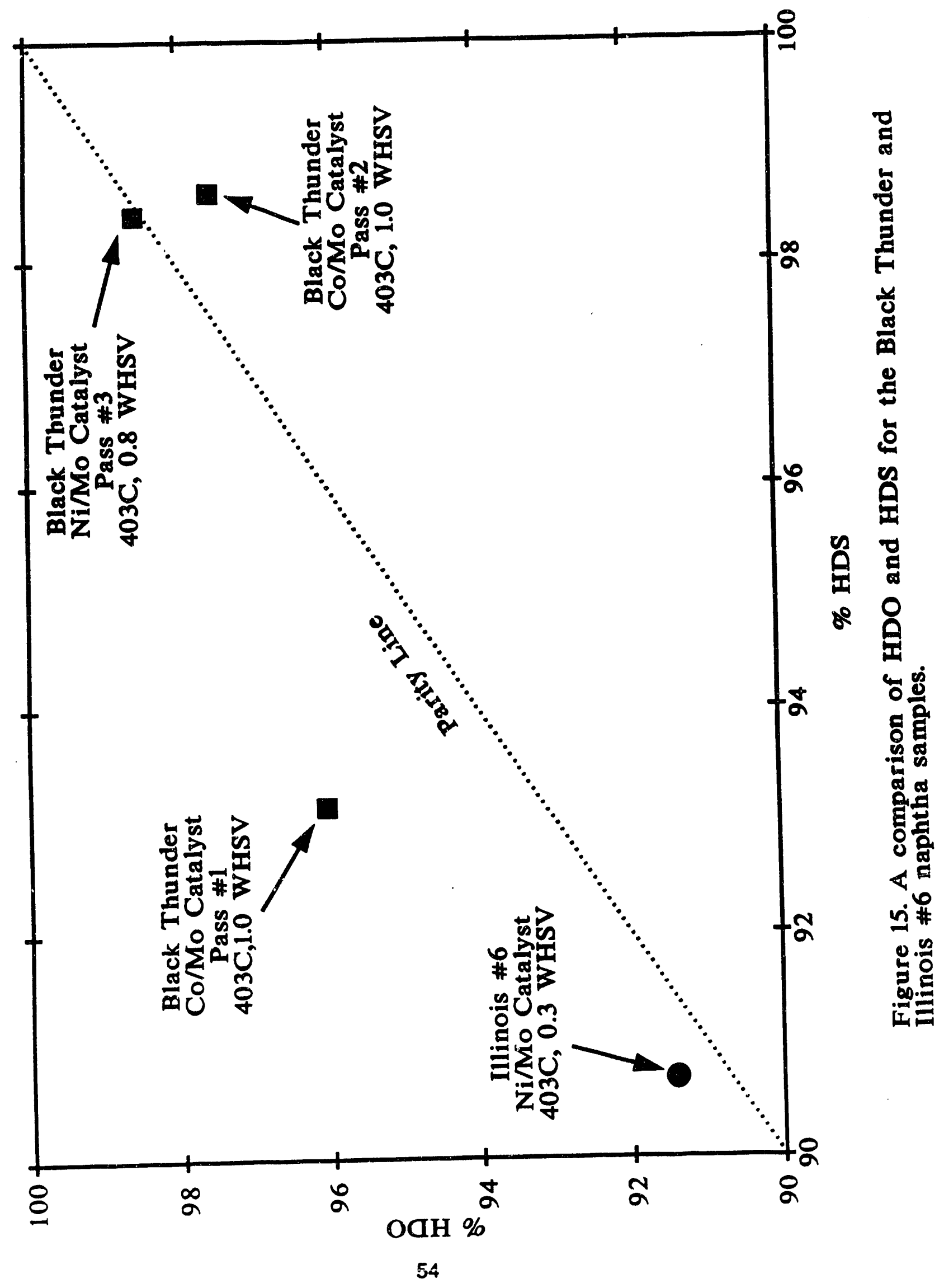




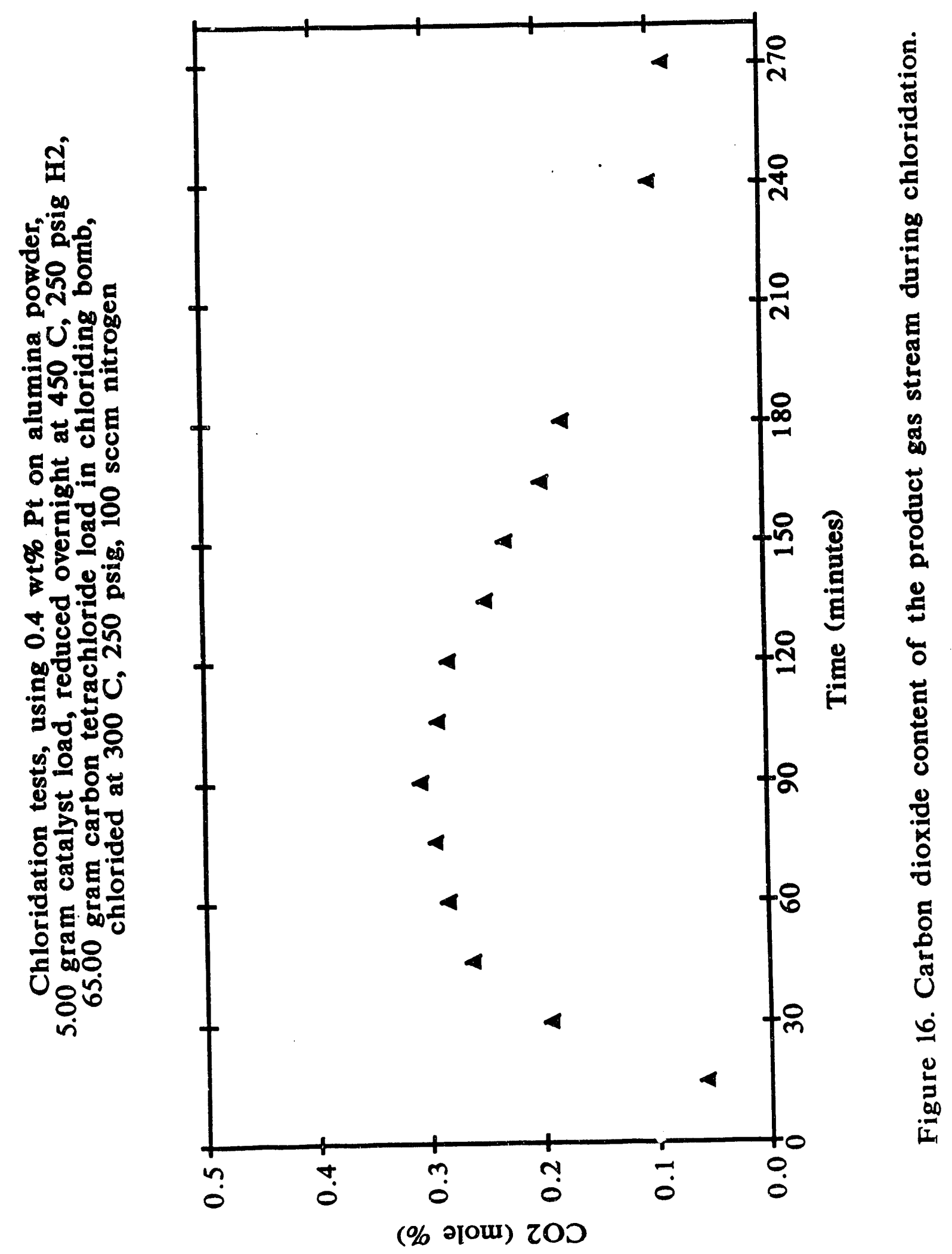




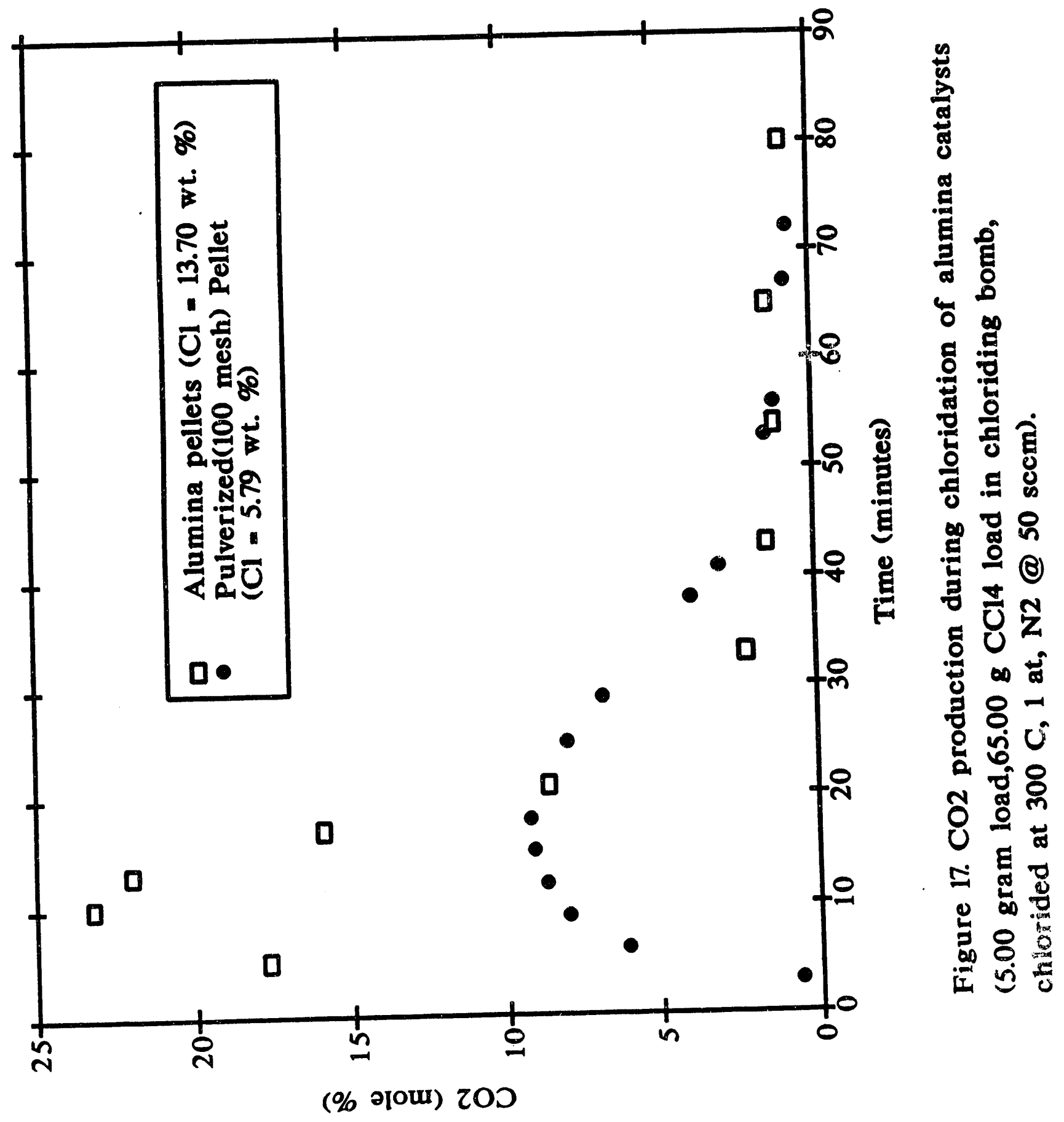




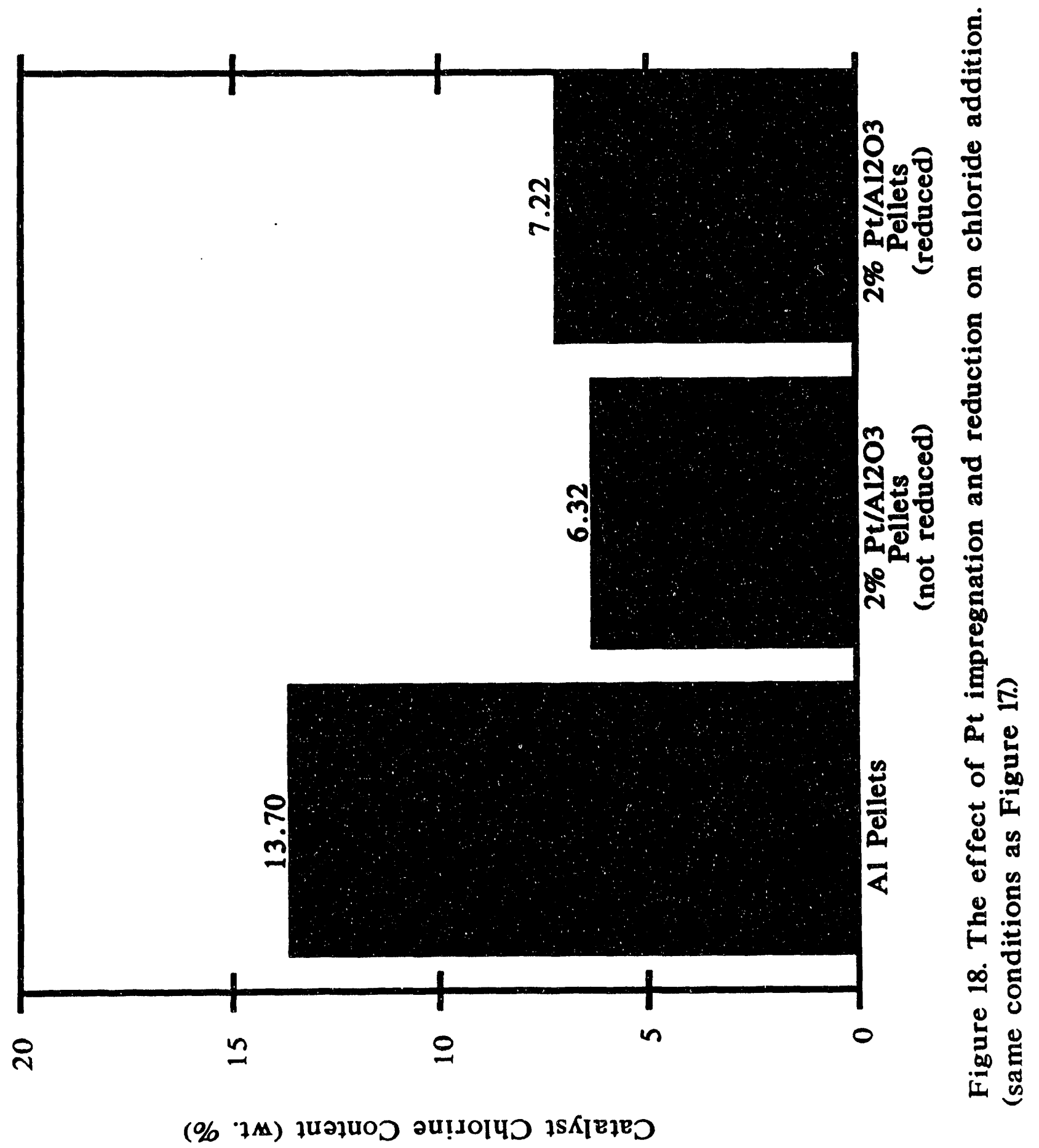




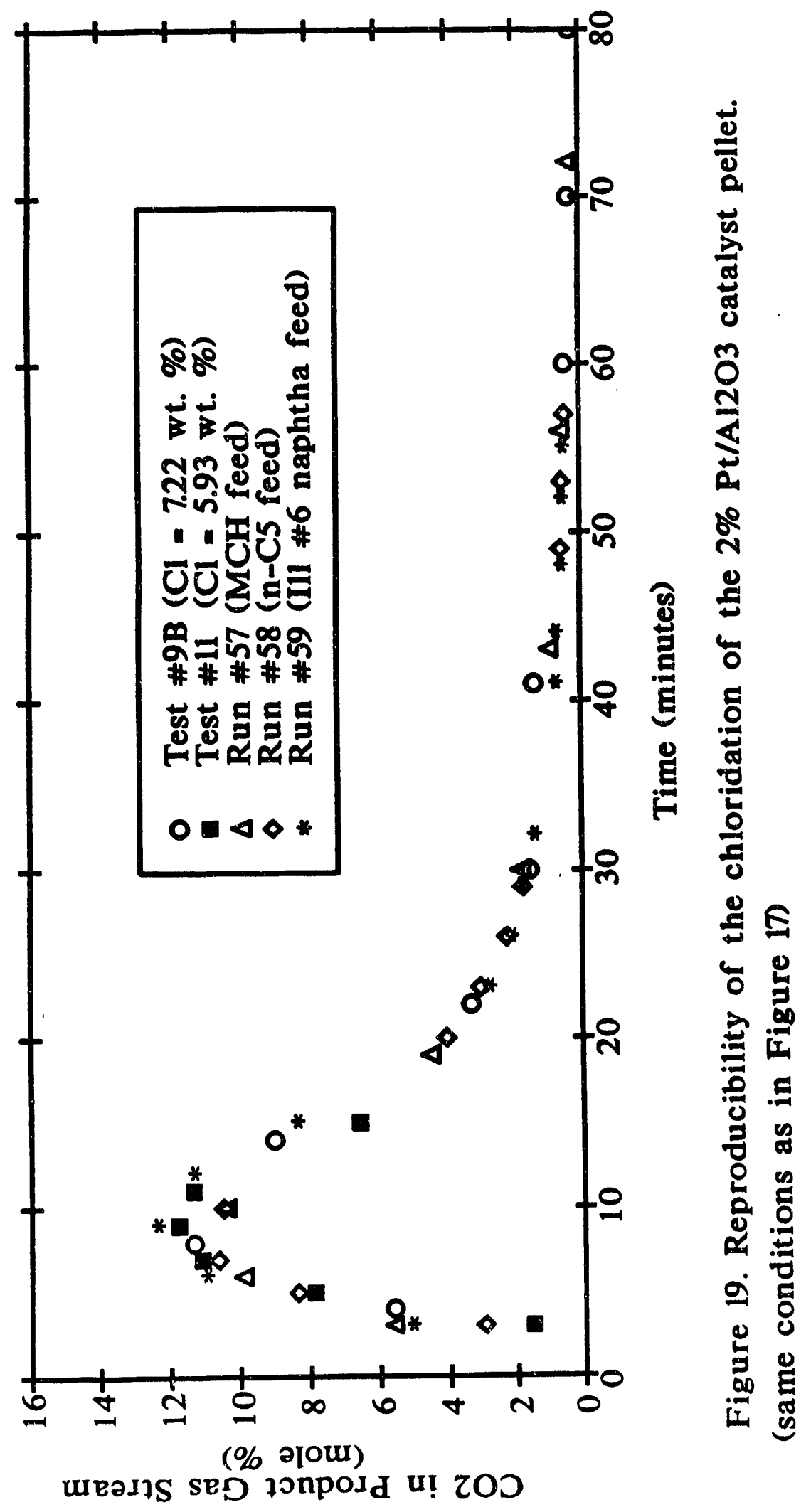




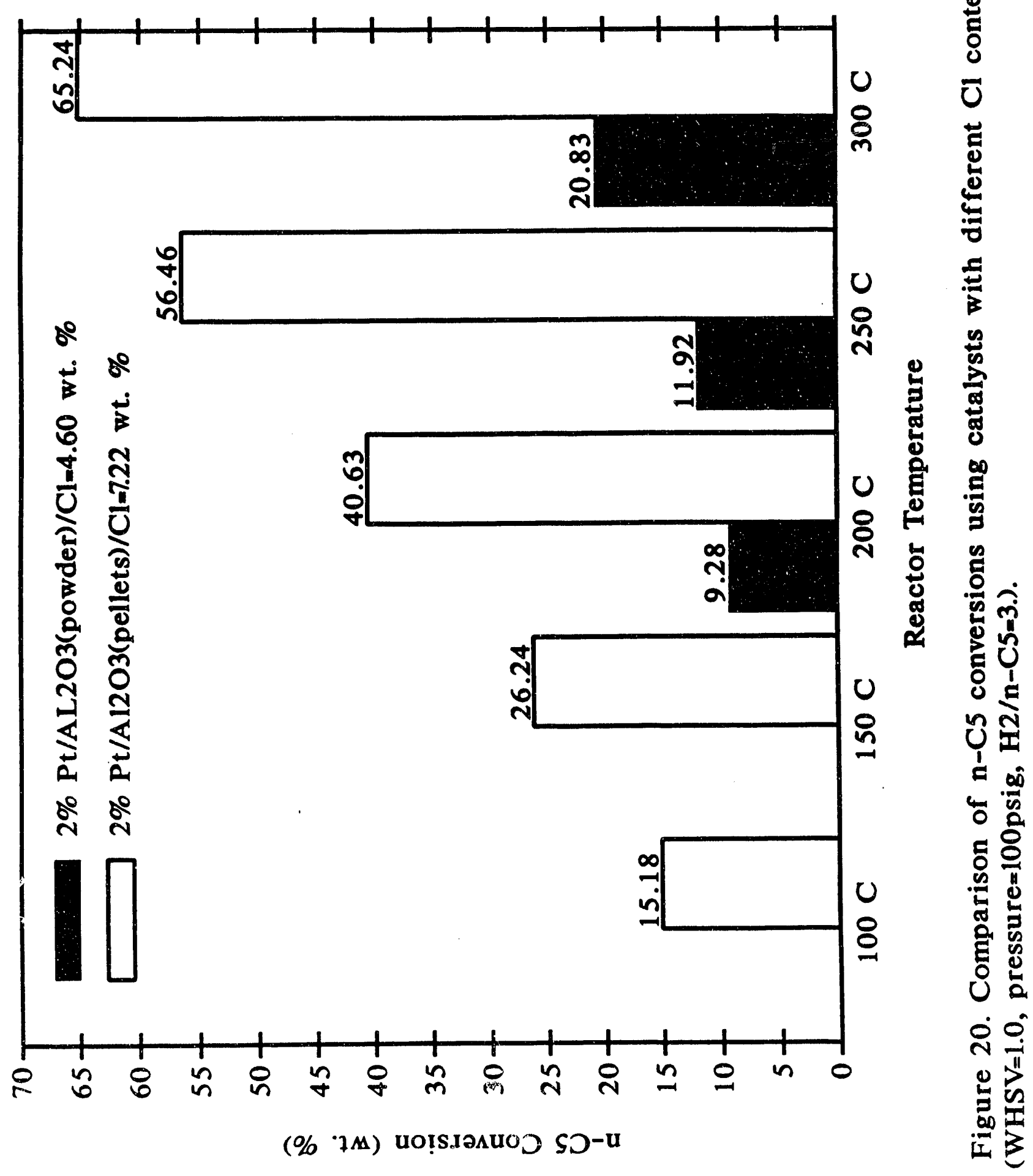




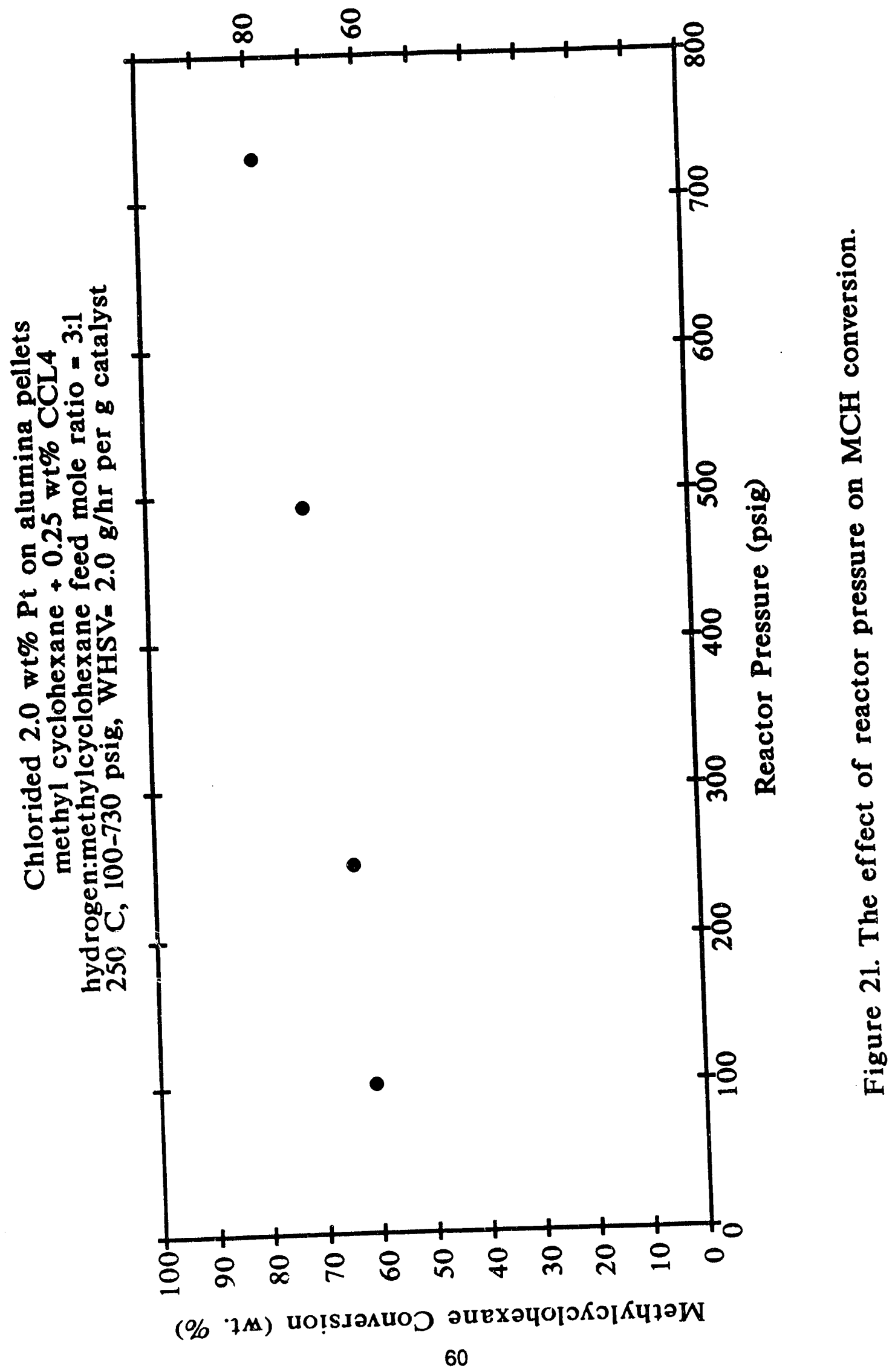




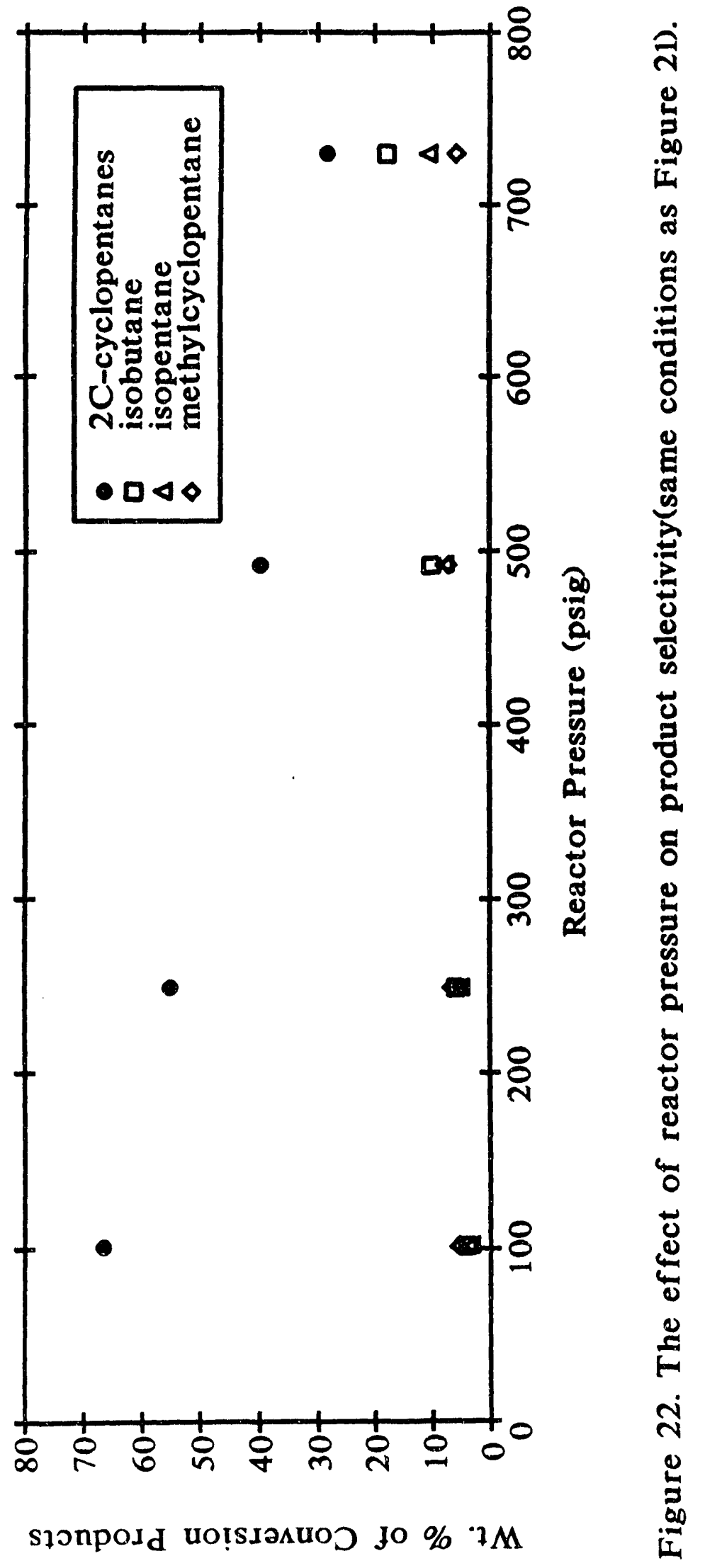




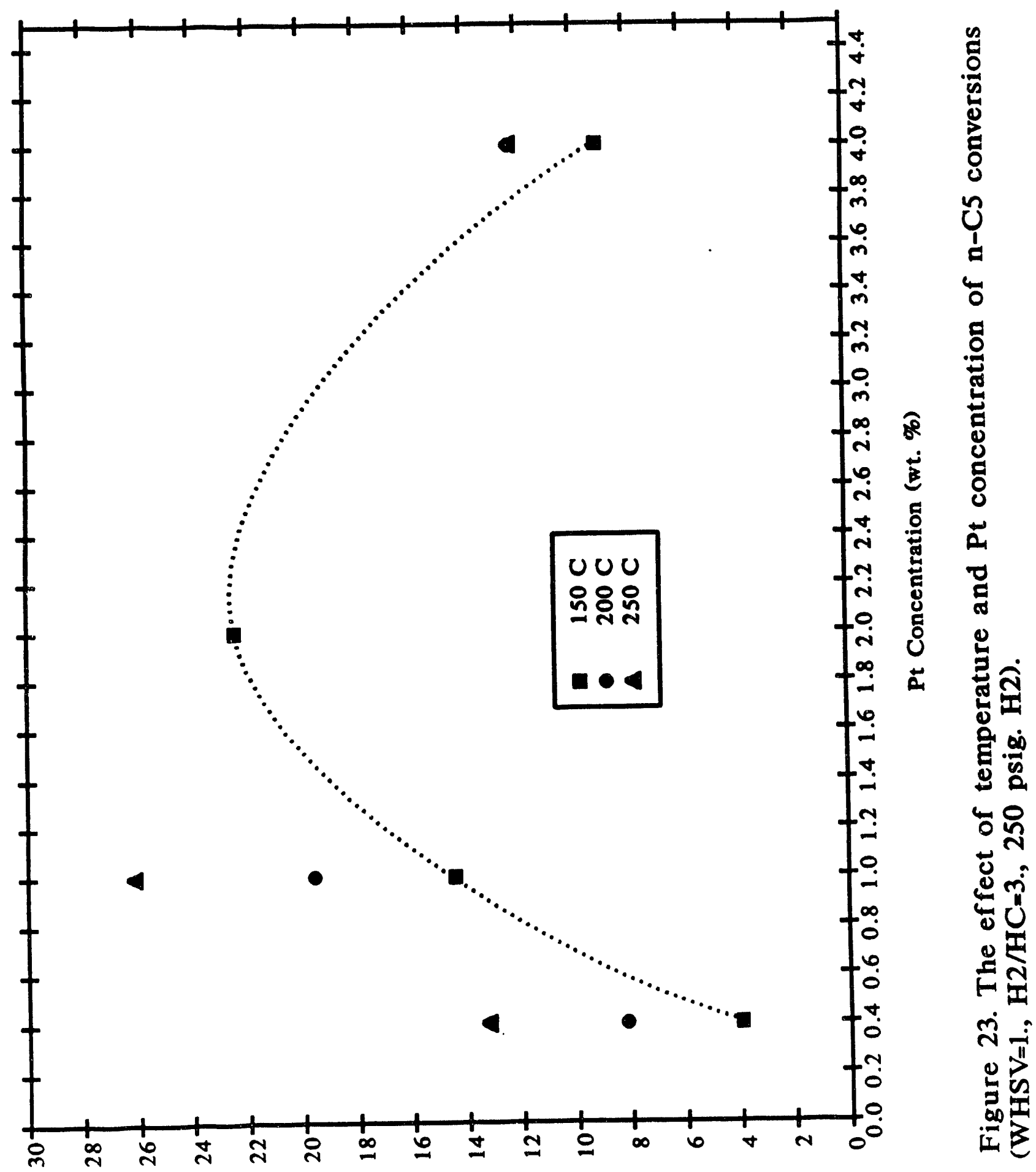

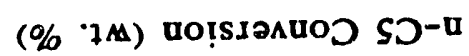




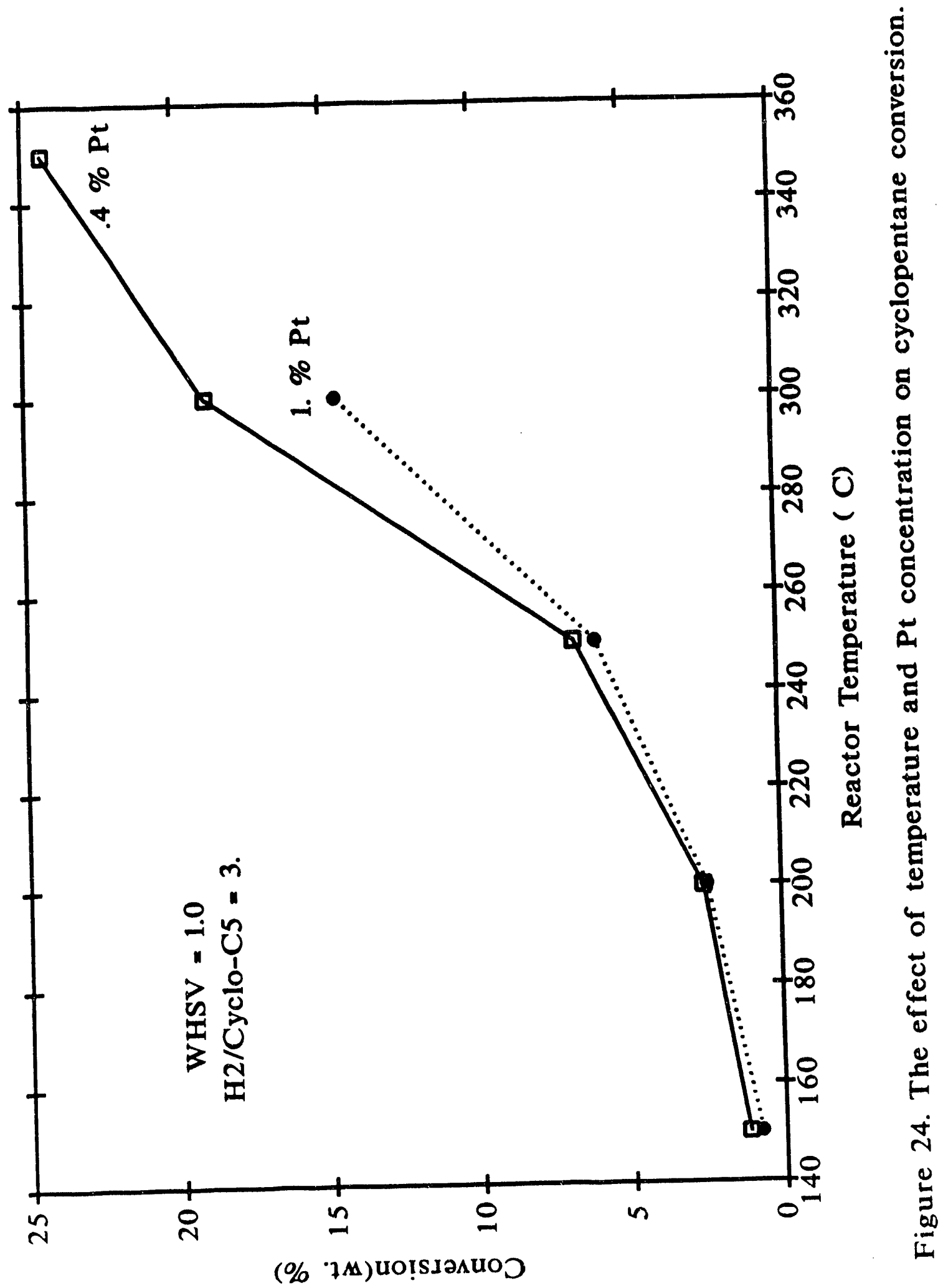




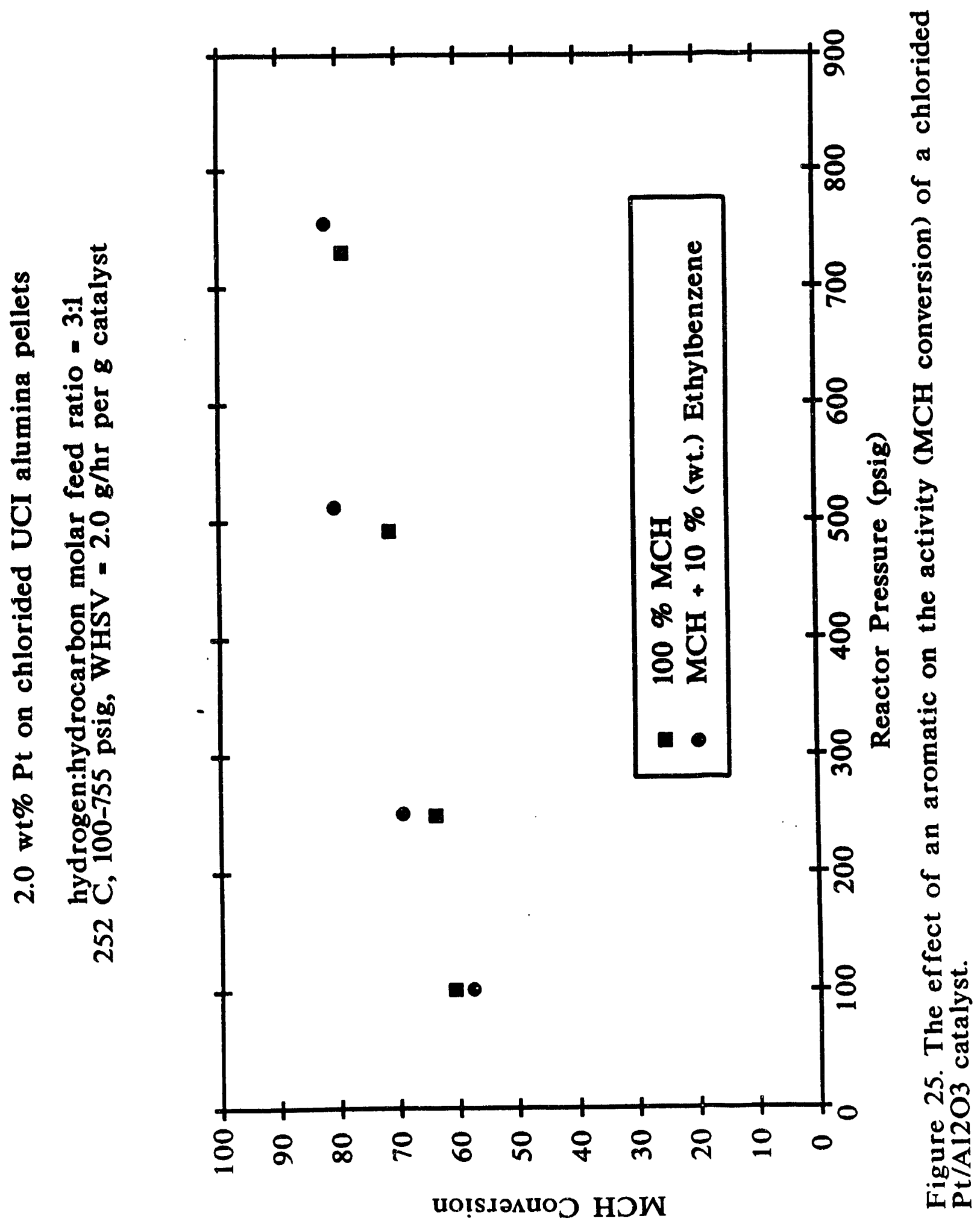




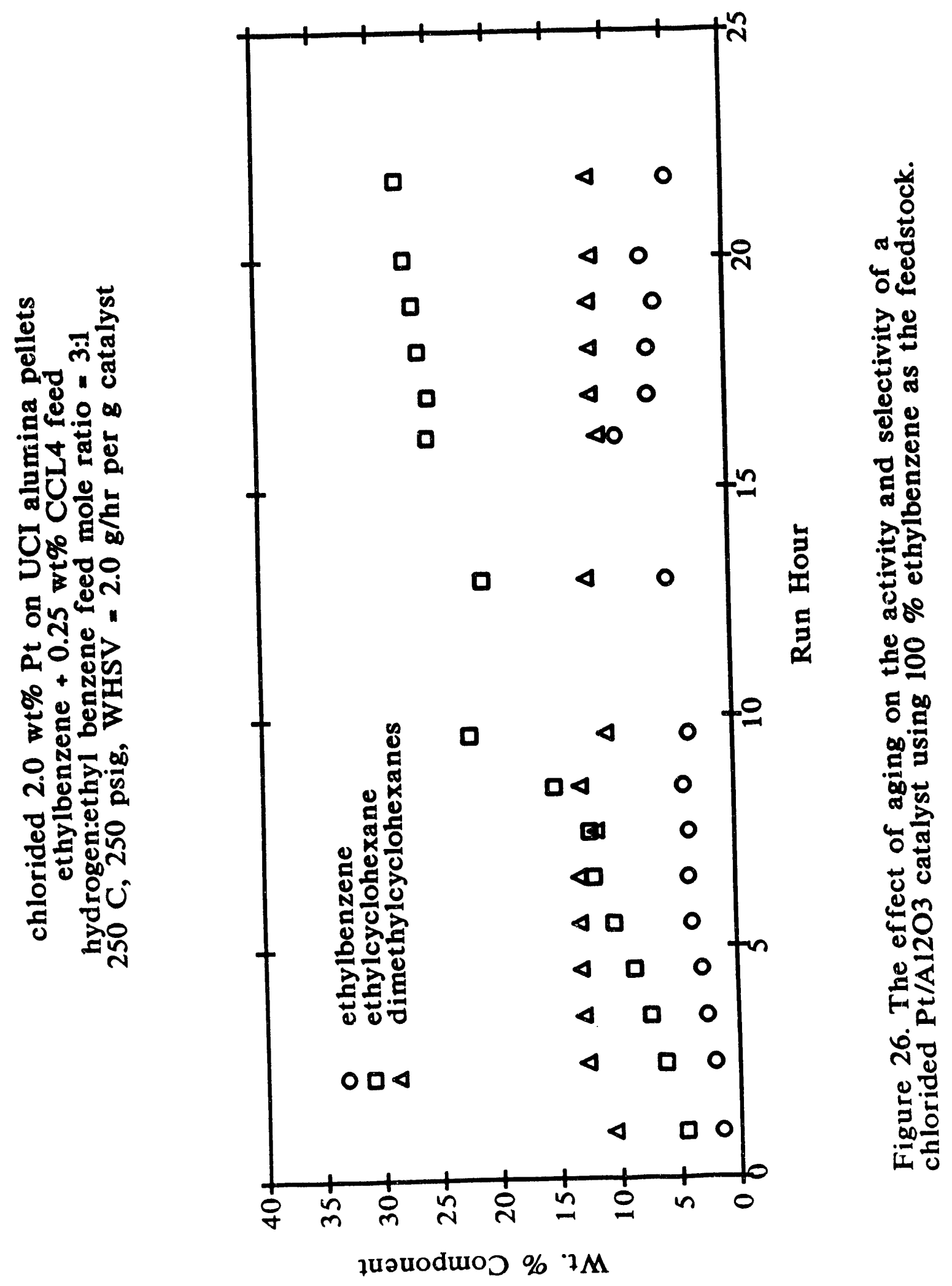




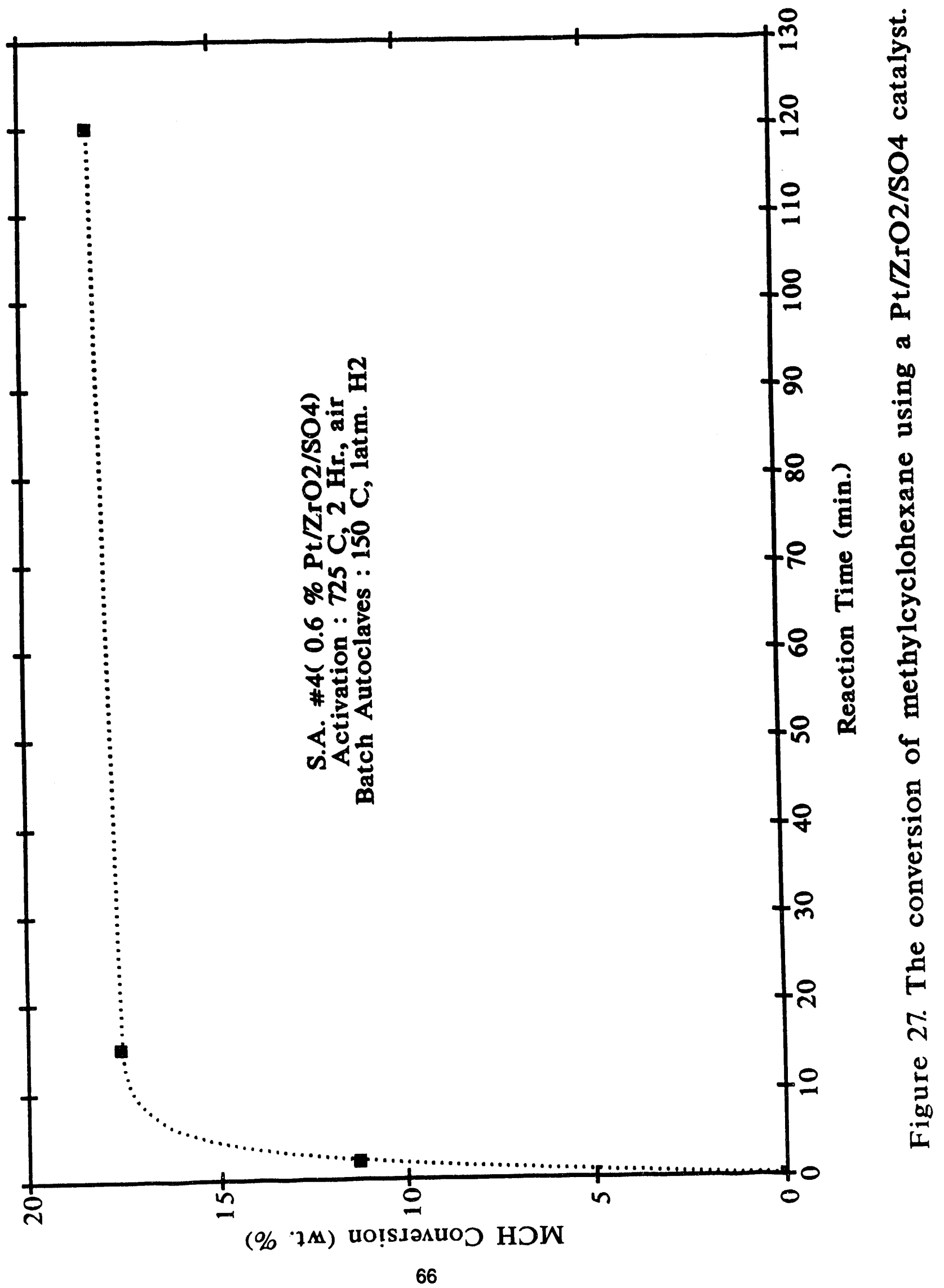




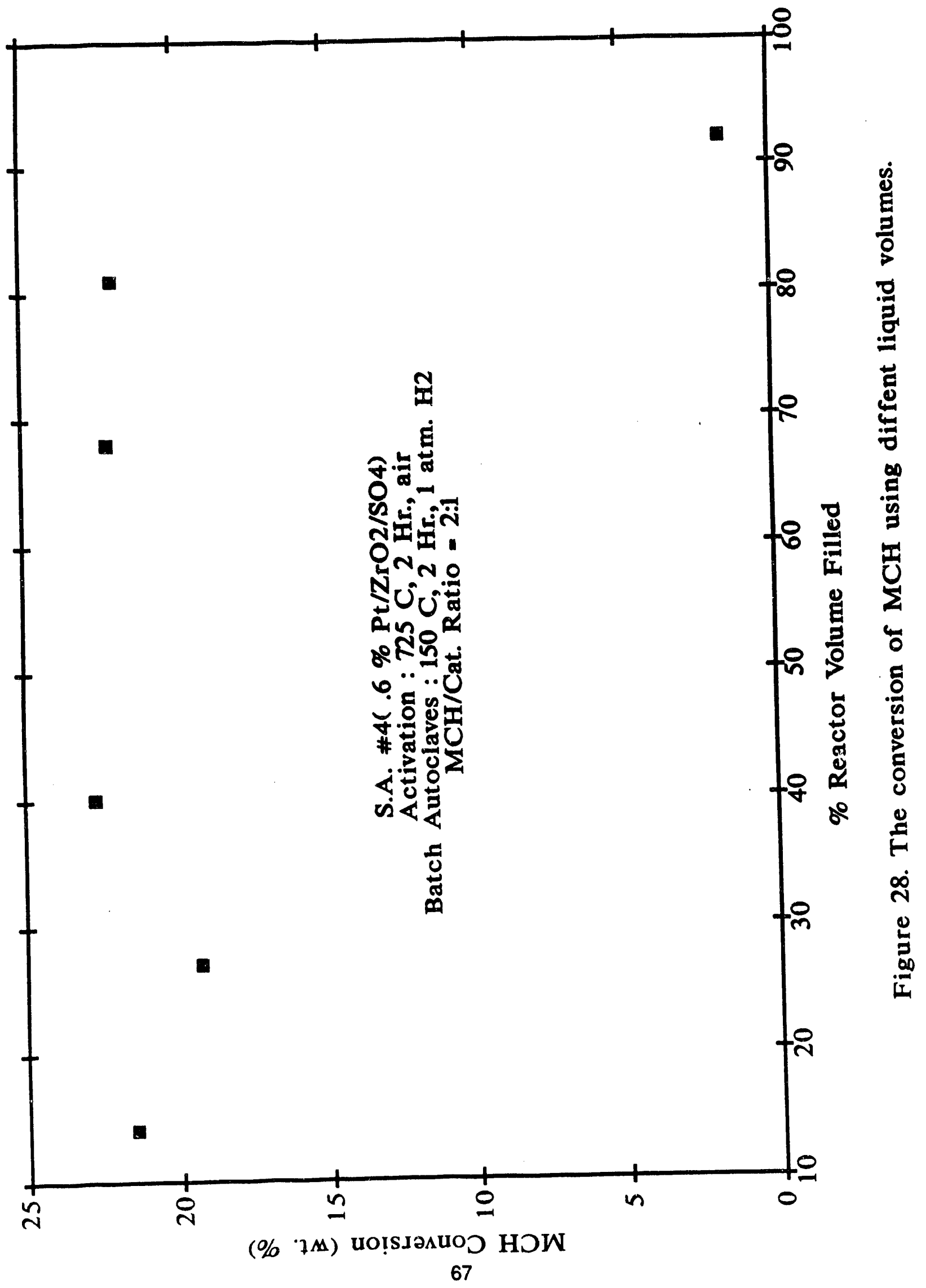




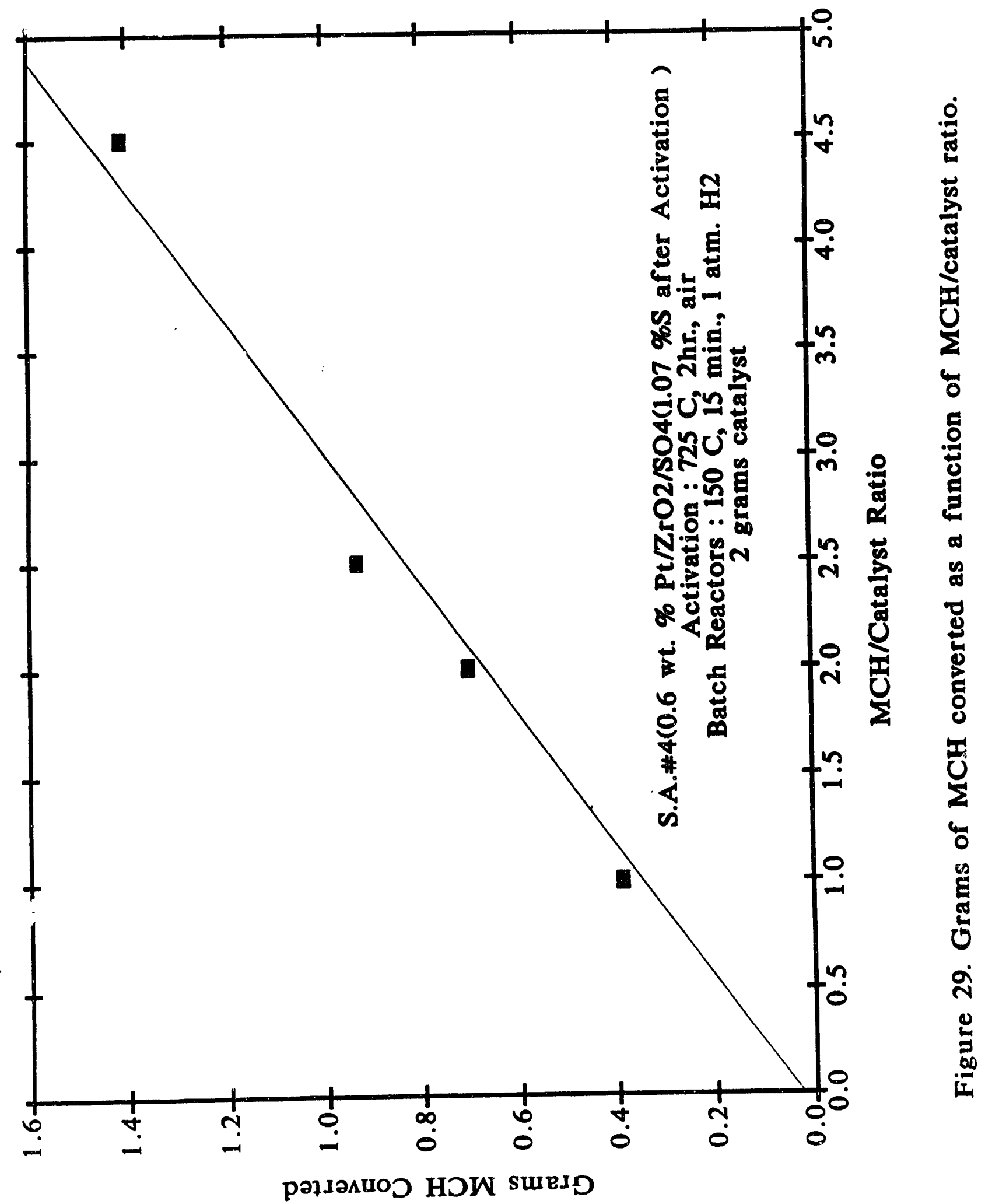




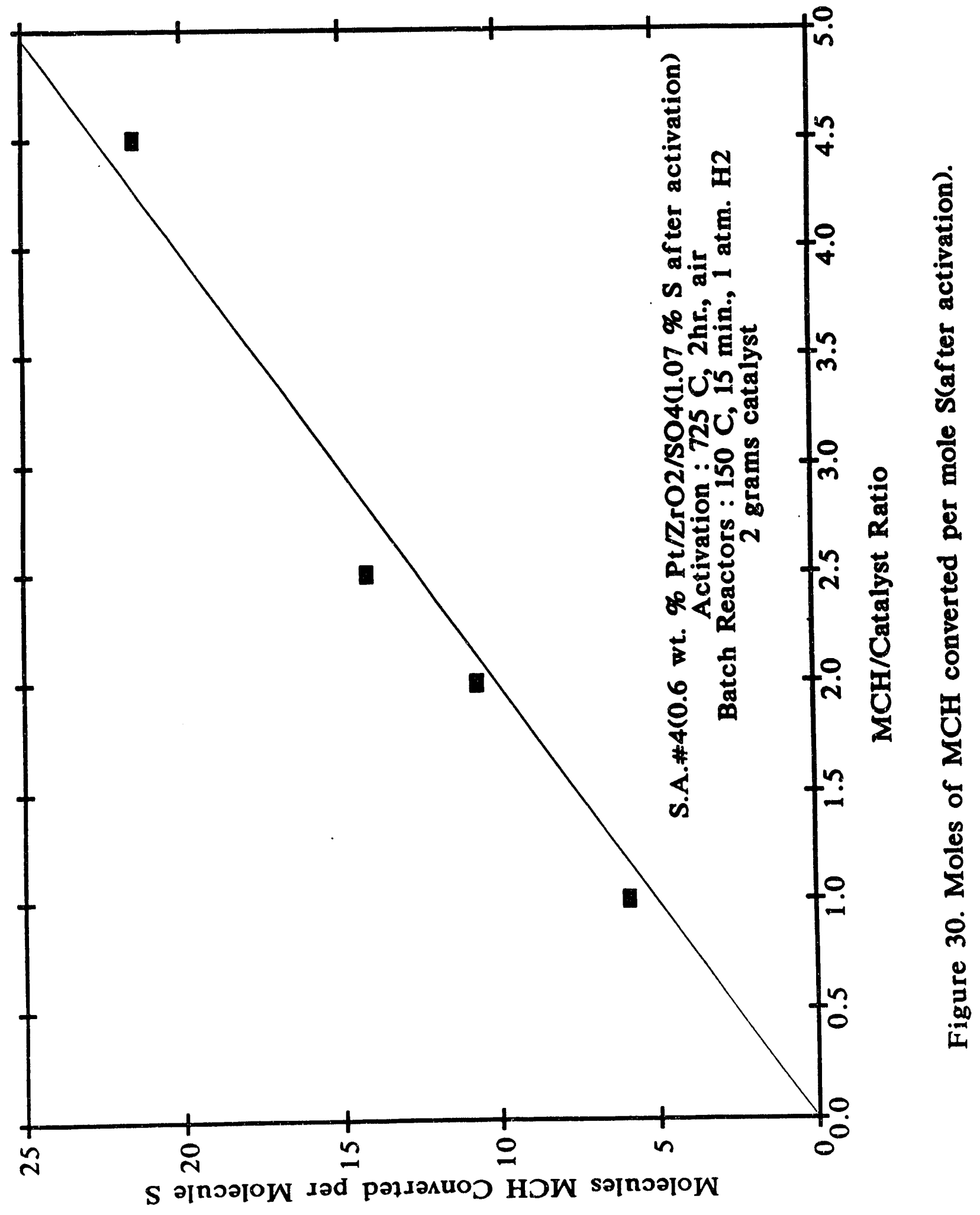




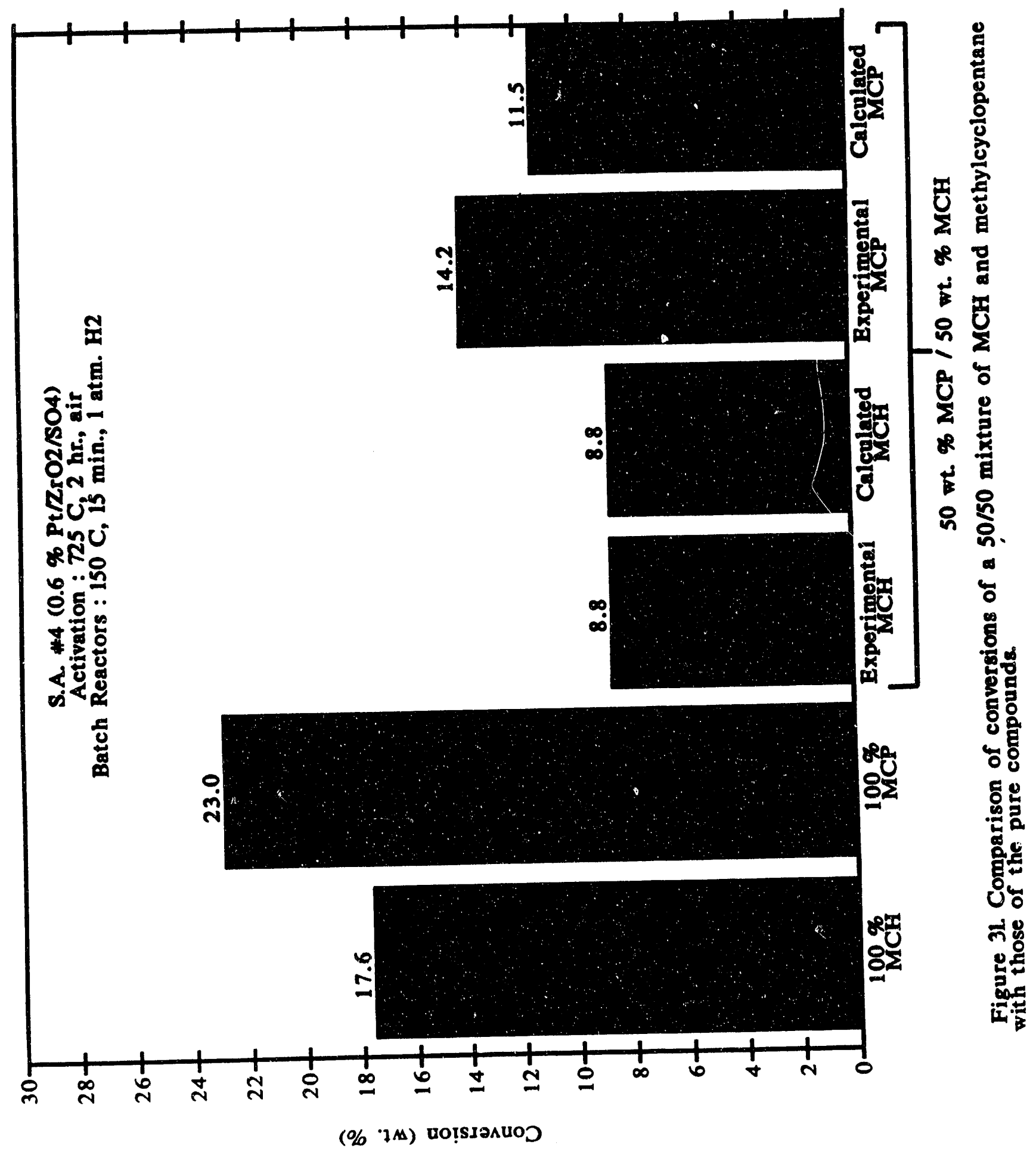




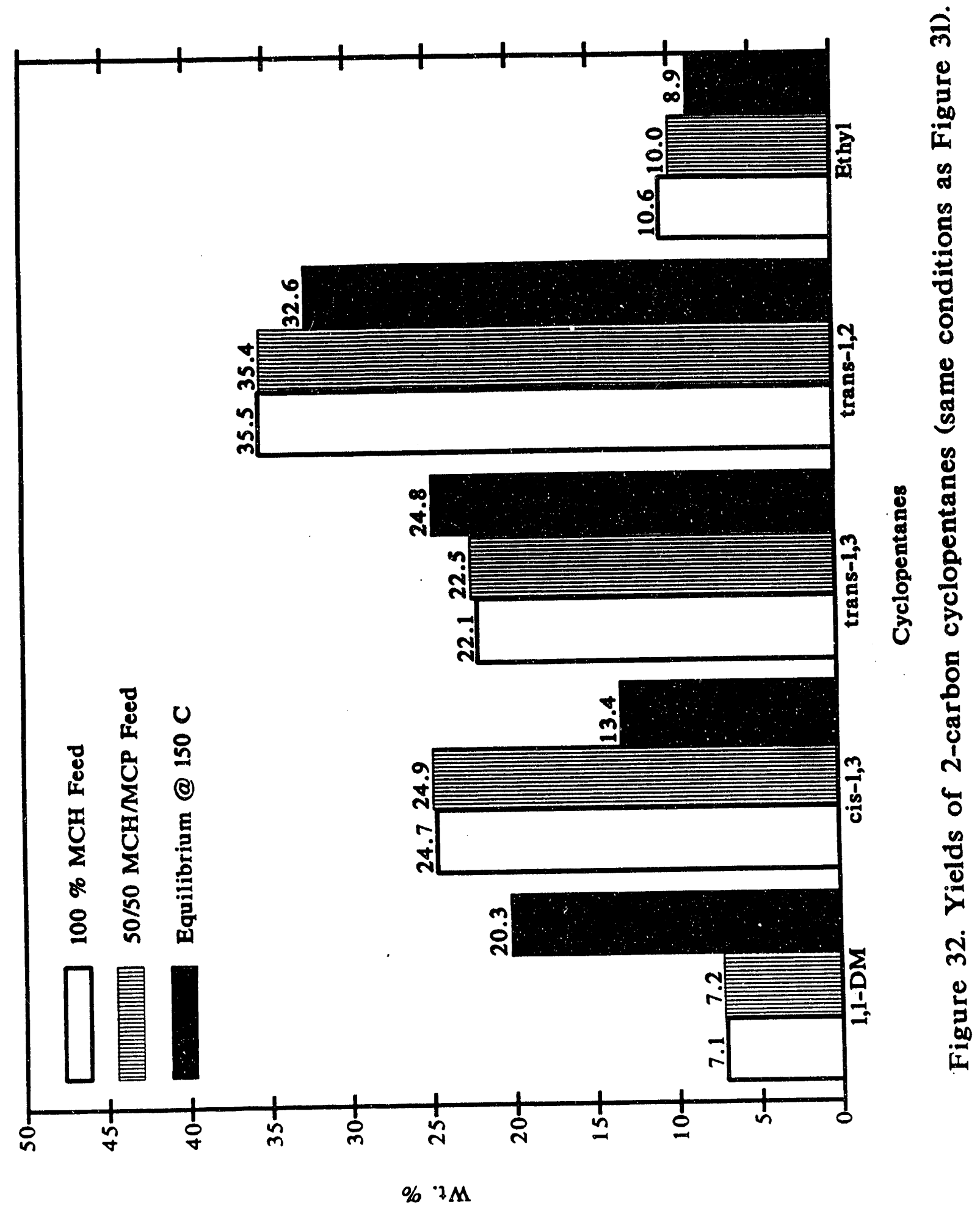




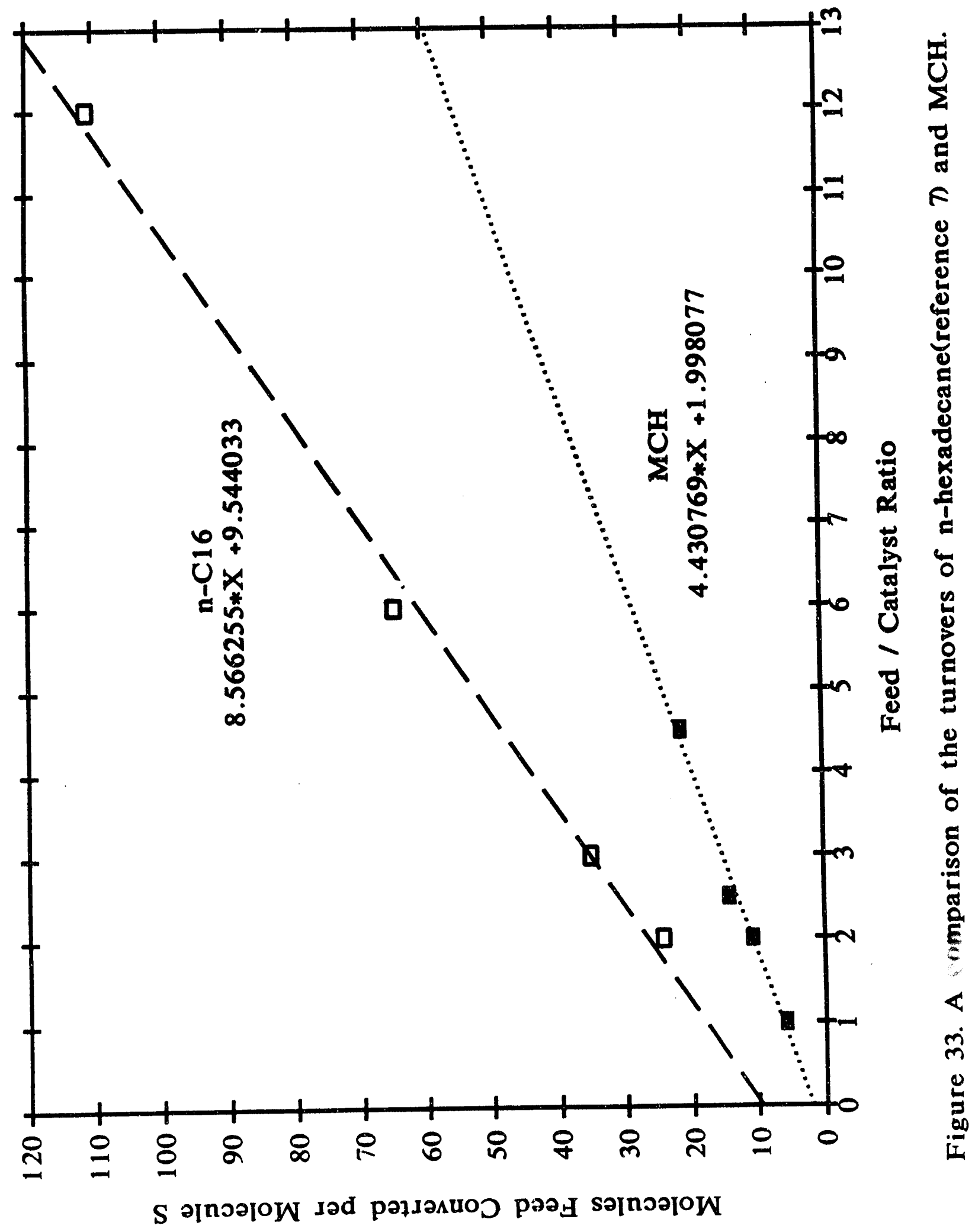




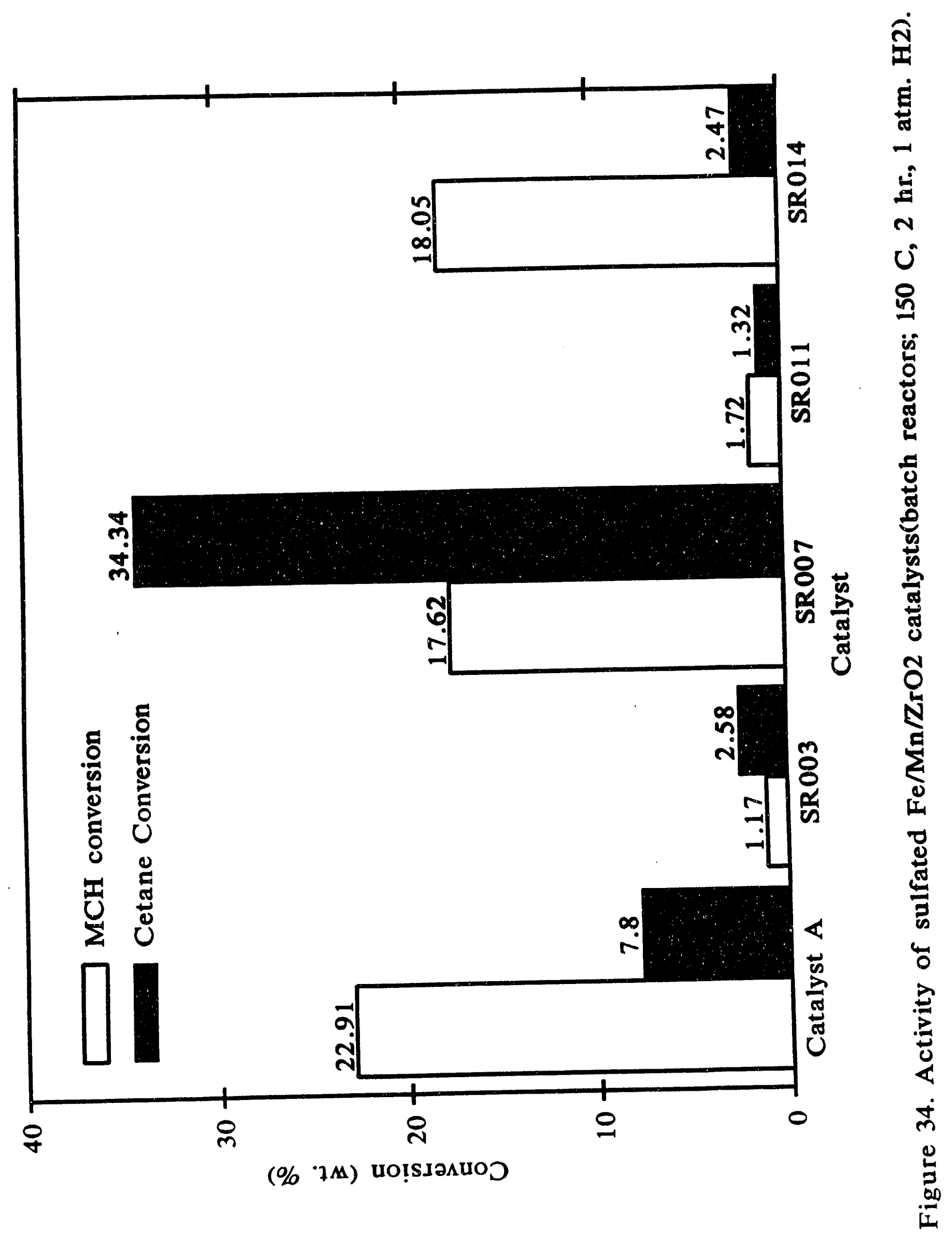




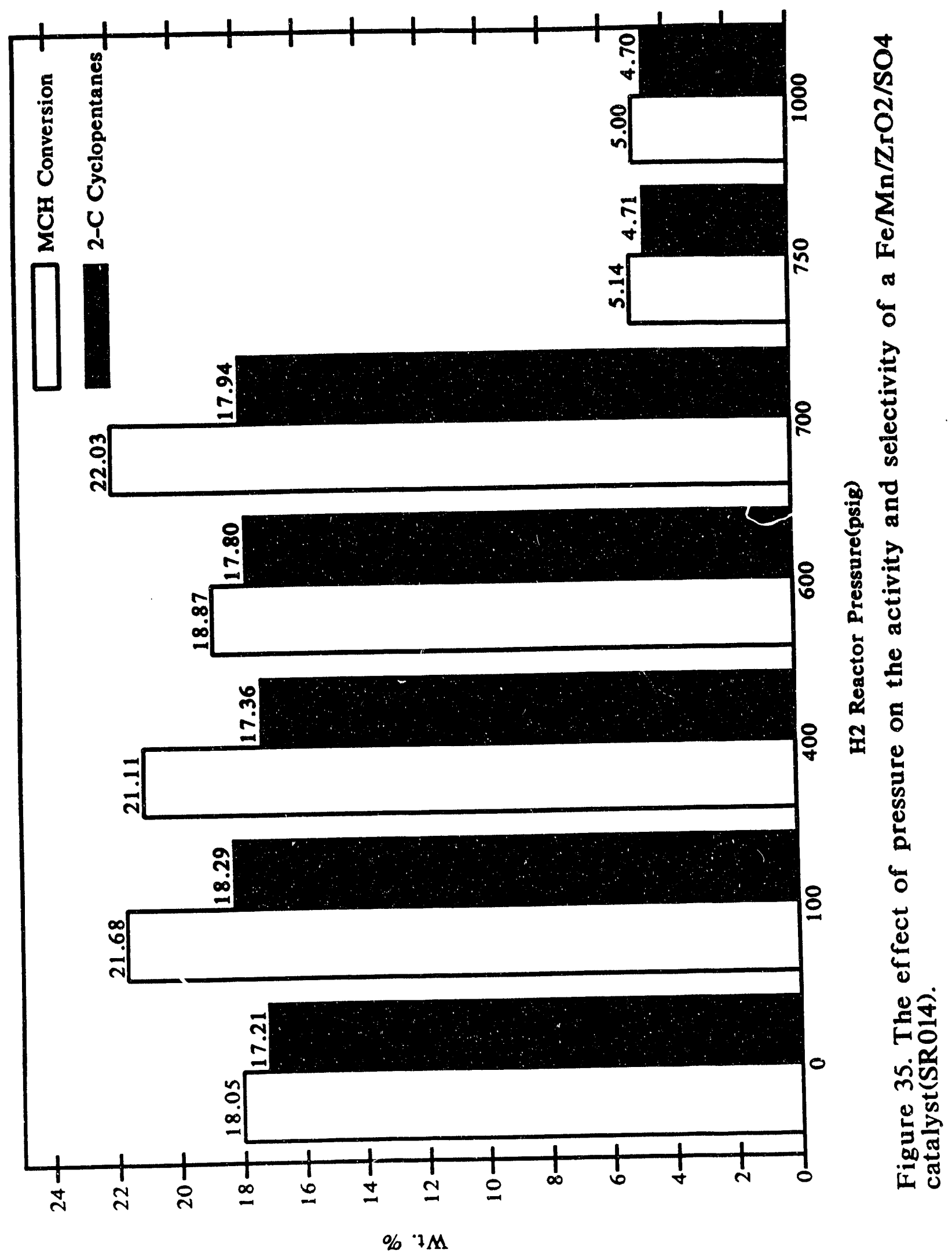




\section{APPENDIX 1}

\section{Summary of Accomplishments:}

1. Identification of the hydrocarbons in the naphtha samples derived from processing an Illinois \#6 (bituminous) and a Black Thunder (subbituminous) coal has been completed. The samples were obtained from the Wilsonville, Alabama coal liquefaction pilot plant. Over 100 compounds have been identified by GC/MS and injection of standard compounds. In addition, the hydrocarbon distributions in the hydrotreated naphtha samples have been determined. The primary difference in the hydrocarbon distribution of the as-received naphtha sample is in the amount of cyclohexanes identified. The total cyclohexane content is larger in the bituminous coal-derived naphtha (26.9 wt.\%) than from the subbituminous naphtha (17.2 wt.\%). Both naphtha samples have one to four carbon substitutions in this hydrocarbon class. Methylcyclohexane is the major component in the bituminous naphtha and the 2-carbon substituted cyclohexanes are the major components found in this class for the subbituminous naphtha.

2. In addition to the hydrocarbon distributions, the nitrogen and sulfur compound distributions were determined using a thermionic detector for nitrogen and a chemiluminescence detector for sulfur installed on capillary gas chromatographs. The major nitrogen components in both naphtha samples were anilines (aniline and 1 to 4-carbon substituted anilines). Quinolines and pyridines were found in approximately equal concentrations in both the naphtha samples. Thiophenes and benzothiophenes were the major sulfur containing compounds found in the naphtha samples. Thiophenes were the major components found in the bituminous coalderived naphtha. Benzothiophenes were the major components in the subbituminous 
coal-derived naphtha. In addition, small concentrations of thiols and sulfides were present in both naphtha samples.

3. The CAER pilot plant was successfully run in the hydrotreating configuration. Three separate runs lasting up to $400+$ hours were completed without a forced shutdown. Two commercially available catalysts (Ni/Mo and Co/Mo) were studied using a number of different process conditions in achieving the target heteroatom compositions of the hydrotreated naphtha samples. The data obtained from these studies suggested that coal-derived naphtha samples will require severe conditions to obtain heteroatom contents of less than $10 \mathrm{ppm}$. For example, data obtained for the three Black Thunder hydrotreating runs suggested that it would require 10 separate passes to obtain a product with a $2 \mathrm{ppm}$ nitrogen content. The element specific detectors (sulfur and nitrogen), which recently became operational, will determine the heteroatomic compounds which remain in the naphtha after hydrotreatment.

4. A method to reproducibly chloride a $\mathrm{Pt}_{\mathrm{A}} \mathrm{Al}_{2} \mathrm{O}_{3}$ catalyst was developed. The chloriding procedure can add from 7 to $13 \mathrm{wt} \% \mathrm{Cl}$ to the catalyst, depending upon the Pt concentration of the catalyst and time of pretreatment, thus producing an active isomerization catalyst. The method employs nitrogen ( $1 \mathrm{~atm}, 100 \mathrm{sccm} / \mathrm{min}$.) as the carrier for $\mathrm{CCl}_{4}$ and a reaction temperature of $300^{\circ} \mathrm{C}$. The extent of chloridation is monitored by determining the amount of $\mathrm{CO}_{2}$ in the gas stream until it is reduced to less the .5 mole \%. The maximum occurs rapidly (ca. $10 \mathrm{~min}$.) and the entire pretreatment requires approximately $40 \mathrm{~min}$. GC/MS analysis of the product gas stream indicated no $\mathrm{COCl}_{2}$ or $\mathrm{CO}$ is produced during this pretreatment.

5. During the development of the method for the chloridation pretreatment step, it was observed that the form of the catalyst significantly affected the amount of 
cinloride added to the catalyst. The initial experiments in the method development employed $\mathrm{Al}_{2} \mathrm{O}_{3}$ with no $\mathrm{Pt}$ added in two forms. An aluminum catalyst, in powder form (-100 mesh) was prepared in-house using standard methods. In addition, a commercially available $\mathrm{Al}_{2} \mathrm{O}_{3}$ pellet was studied. The chloriding pretreatment step, for both samples, used the same catalyst loads and pretreatment conditions. The resulting $\mathrm{CO}_{2}$ concentration versus time curves were distinctly different. For the powdered $\mathrm{Al}_{2} \mathrm{O}_{3}$ catalyst, the maximum $\mathrm{CO}_{2}$ generated was much lower ( 1 mole \%) and occurred at a longer reaction time $\left(20 \mathrm{~min}\right.$.) when compared to that of the $\mathrm{Al}_{2} \mathrm{O}_{3}$ pellets which produced a maximum of 22 mole $\% \mathrm{CO}_{2}$ in $10 \mathrm{~min}$. The amount of $\mathrm{Cl}$ added to the powdered catalyst was significantly lower than that of the pellets. In order to confirm that the form of the catalyst was responsible for the difference in the amount of chlorided added to the two catalysts, the pellets were ground to -100 mesh and the chloridation pretreatment was repeated. The $\mathrm{CO}_{2}$ generation curve produced by the "powdered" pellet catalyst was very similar to that of the powdered catalyst prepared in-house.

One possible explanation for these data is that for the alumina pellets, the $\mathrm{CCl}_{4}$ penetrates the pellets and reacts rapidly with the alumina to produce a chlorided alumina compound and $\mathrm{CO}_{2}$. The penetration of the $\mathrm{CCl}_{4}$ into the pellet and the escape of the $\mathrm{CO}_{2}$ out of the pellet was not thought to be diffusion limited. It is believed that the diffusion of resulting $\mathrm{AlCl}_{3}$ from the interior of the catalyst particle is slow due to diffusion or a process that resembles diffusional limitations. Thus it is viewed that the $\mathrm{AlCL}_{3}$ forms uniformly throughout the catalyst particle or pellet, but can be lost from the catalyst bed by desorption from the surface boundary of the catalyst. The geometric surface boundary of the pellet is much smaller than that of the same 
amount of catalyst when it is present in powder form. Therefore, based on the data obtained to date, the rate of chloridation is similar in both the powder and the pellet; however, desorption of the gas in the void volume and transport from the catalyst bed is much smaller for the pellet than for the powder.

6. A chlorided $\mathrm{Pt}(2 \%) / \mathrm{Al}_{2} \mathrm{O}_{3}$ (pellet) catalyst produced high conversions of methylcyclohexane $(58 \mathrm{wt} . \%)$ using relatively mild conditions $\left(250^{\circ} \mathrm{C}, \mathrm{WHSV}=1,100\right.$ psig $\mathrm{H}_{2}$ ). However, the selectivity to isoalkanes was very low. The primary products were 2-carbon cyclopentanes. The selectivity to isoalkanes did not significantly increase by increasing the temperature. Increasing the hydrogen pressure (at $250^{\circ} \mathrm{C}$, WHSV = 1) from 100 psig to 735 psig increased the conversion of methylcyclohexane from 58 to $79 \mathrm{wt}$ \% and significantly improved the selectivity. The amount of 2-carbon cyclopentanes was reduced from 69 to $30 \mathrm{wt}$.\% of the conversion products with a concurrent increase in isoalkane production. This data suggested that to effect ring opening of the cycloalkanes, higher pressures will be required.

7. The chlorided $\mathrm{Pt}(2 \%) / \mathrm{Al}_{2} \mathrm{O}_{3}$ (pellet) catalyst was studied using a $10 \mathrm{wt} . \%$ ethylbenzene/methylcyclohexane to determine if the aromatics in a naphtha deactivates the catalyst and if the aromatics can be hydrogenated to a cycloalkane followed by ring opening. The feedstock was run using the same conditions previously used for the $100 \%$ methylcyclohexane $\left(250^{\circ} \mathrm{C}\right.$, WHSV $=1$, pressure $=100$ - 750 psig $\mathrm{H}_{2}$ ). The results of the run indicated no appreciable difference in the deactivation of the catalyst with the aromatic/methylcyclohexane feed. The data also indicated that with time on stream, the amount of the aromatic converted to the cycloalkane did not significantly decrease. The amount of the $\mathrm{C}_{8}$ cycloalkane converted to isoalkane also did not significantly decrease with time on stream. It 
appears that from these data that ring opening of the $\mathrm{C}_{8}$ cycloalkane (ethylcyclohexane) is effected more easily than the $\mathrm{C}_{7}$ cycloalkane (methylcyclohexane).

A $100 \%$ ethylbenzene feedstock was converted using a freshly chlorided $\mathrm{Pt}_{\mathrm{A}} \mathrm{I}_{2} \mathrm{O}_{3}$ (pellet) catalyst to confirm the results discussed above. The product distribution produced was exactly as expected; however, using the $100 \%$ aromatic feed did deactivate the catalyst more rapidly. Conversion of the ethylbenzenes did not significantly decrease with time on stream; however, the ring-opening of the corresponding cycloalkane did decrease with catalyst age. These data suggest that the metal function was not deactivated and the acid function was deactivated with time on stieam. This is consistent with previous data obtained using 1-octene as the feedstock at the completion of a number of runs.

8. A large number of sulfated $\mathrm{Zr}$-based catalysts have been prepared. The catalysts include Pt impregnated, $\mathrm{Fe}$ and $\mathrm{Mn}$ coprecipitated and catalysts prepared from different precursor salts of zirconium. As a minimum, all of the catalysts prepared have been tested for activity in batch autoclaves.

9. A number of different activation temperatures have been studied using the sulfated $\mathrm{Zr}$ catalysts. To date, the best conversions obtained with methylcyclohexane were obtained using a temperature of $725^{\circ} \mathrm{C}$ for 2 hours and an air atmosphere. At this activation temperature a substantial amount of the sulfur is lost; however, the conversions obtained using methylcyclohexane do not appear to depend on the amount of sulfur remaining after activation. The data suggested that the amount of sulfur initially added is not as important as how the sulfur is incorporated into the matrix after activation in producing an active catalyst. 
10. A number of different $Z r$-based catalyst have been tested in the plug-flow reactors constructed for this research using methylcyclohexane and n-pentane as feedstocks and a number of process conditions. During the runs using reaction temperatures greater than $200^{\circ} \mathrm{C}$, it was observed that $\mathrm{H}_{2} \mathrm{~S}$ was being formed indicating that sulfur was being removed from the sulfated $\mathrm{Zr}$ catalysts and may be the cause of loss of activity. Decreasing the partial pressure of hydrogen did not significantly increase the conversion of methylcyclohexane or decrease the rate of deactivation. The selectivity to isoalkanes of the zirconia catalysts run in both the batch and plug-flow reactor run was low. The major products were 2-carbon cyclopentanes. Increasing the total pressure of the system did not increase the amount of ring-opening of the cycloalkanes. 


\section{APPENDIX 2}

\section{List of Project Presentations and Publications}

1. Xu, L., Keogh, R. A., Huang, C. S., Spicer, R. L., Sparks, D. E., Lambert, S., Thomas, G. A., and Davis, B.H., "Catalytic Hydrotreatment of Coal-Derived Naphtha, Am. Chem. Soc., Div. Fuel Preprints, 36 (4), 1909 (1991).

2. R. Srinivasan and B. H. Davis, "Sulfated Zirconia Superacid Catalysts: Thermal Analysis and Characterization Studies", Am. Chem. Soc., Div. Petro. Prepr., 36 (4), 635 (1991).

3. R. A. Keogh, D. E. Sparks, G. A. Thomas and B. H. Davis, "Chloridation and Activity of $\mathrm{Pt}^{\mathrm{A}} \mathrm{I}_{2} \mathrm{O}_{3}$ Catalysts", Am. Chem. Soc., Div. Fuel Prepr., ACS Meeting, Washington, DC, 1992.

4. R. A. Keogh, B. Shi, S. J. Liaw, A. Fort and B. H. Davis, "Compositional Differences in Naphtha Derived from Non-Conventional Fossil Fuels", Am. Chem. Soc., Div. Fuel Chem. Prepr., ACS Meeting, Washington, DC, 1992.

5. L. Xu, R. A. Keogh, C. S. Huang, R. L. Spicer, D. E. Sparks, S. Lambert, G. A. Thomas and B. H. Davis, "Catalytic Hydrotreatment of Coal-Derived Naphtha", submitted to Fuel Proc. Tech.

6. R. Srinivasan and B. H. Davis, "Crystallization Exotherms of $\mathrm{ZrO}_{2}-\mathrm{HfO}_{2}$ Mixtures", J. Am. Ceram. Soc., 73, 1780-1782 (1990).

7. R. Srinivasan, L. Rice and B. H. Davis, "Critical Particle Size and Phase Transformation in Zirconia: TEM and XRD Studies", J. Am. Ceram. Soc., 73 [11], 3528-3530 (1990). 
8. R. Srinivasan, R. J. De Angelis, G. Ice and B. H. Davis, "Identification of Tetragonal and Cubic Structures of Zirconia", J. Mater. Res., J. Mater. Res., 6 (6), 1287-1292 (1991).

9. R. Srinivasan, S. F. Simpson, J. M. Harris and B. H. Davis, "Discrepancies in the Crystal Structures Assigned to Precipitated Zirconia", J. Mater. Sci. Lett., 10 (6), 352-354 (1991).

10. R. Srinivasan, D. Taulbee and B. H. Davis, "The Effect of Sulfate on the Crystal Structure of Zirconia", Catal. Lett., 9, 1-8 (1991).

11. R. Srinivasan, B. H. Davis, L. Rice and R. J. De Angelis, "Electron Microdiffraction Studies of Zirconia Particles", J. Mater. Sci., 27, 661-670 (1992).

12. R. Srinivasan, B. H. Davis, O. B. Cavin and C. R. Hubbard, "Crystallization and Phase Transformation Process in Zirconia: An in-situ High Temperature X-ray Diffraction Study", J. Am. Ceram. Soc., 75 (5), 1217-1222 (1992).

13. R. Srinivasan and B. H. Davis, "Influence of Zirconium Salt Precursors on the Crystal Structures of Zirconia", Caral. Lett., in press.

14. R. A. Keogh and B. H. Davis, "Sulfated Zirconia: Is Superacidity Reponsible for its Hydrocarbon Isomerization Activity?", submitted to Catal. Lett. 

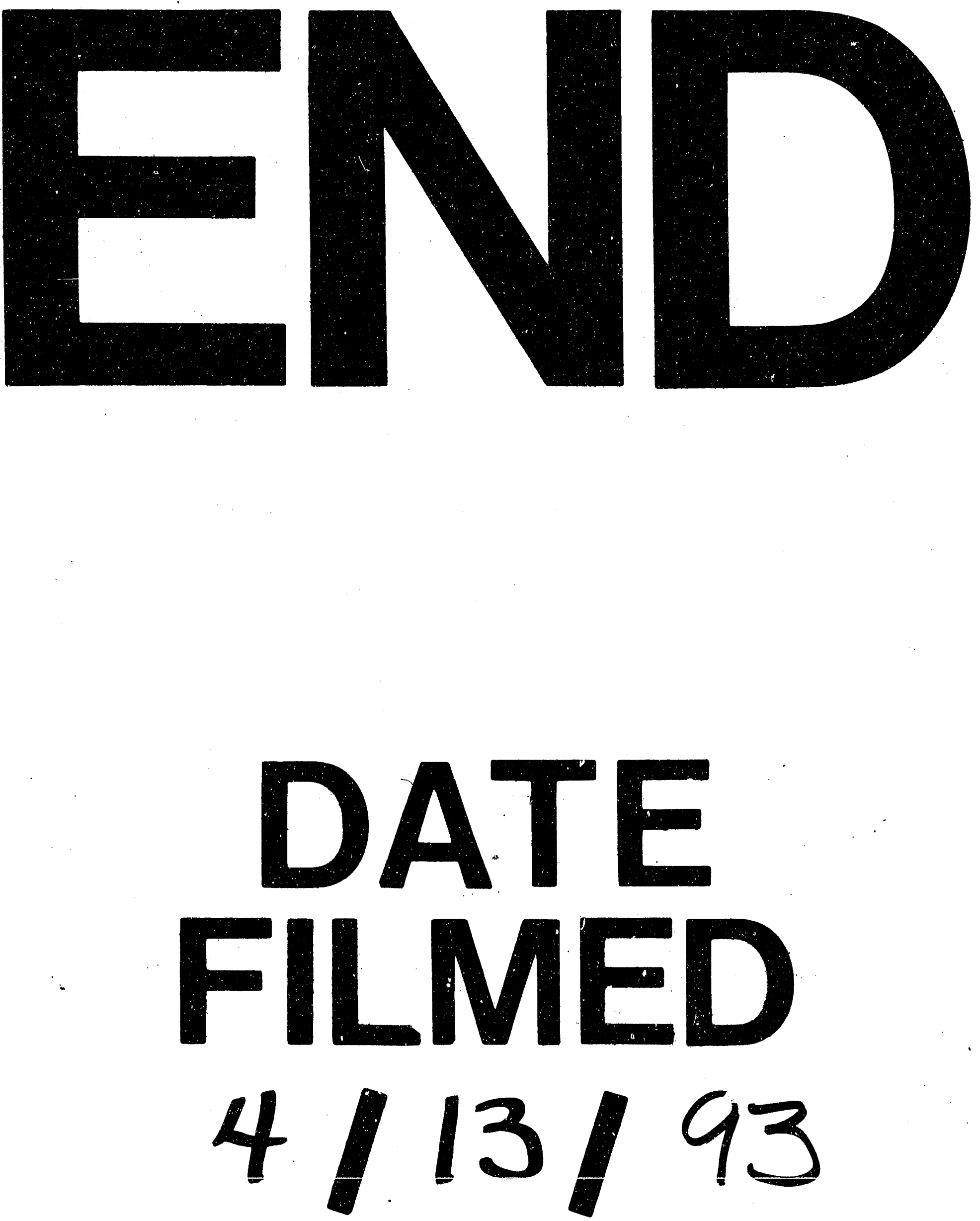

1 
